UNIVERSIDADE DE SÃO PAULO

ESCOLA DE EDUCAÇÃO FÍSICA E ESPORTE

ESTUDO DOS EFEITOS DA PRÁTICA DE IOGA NA FREQÜÊNCIA CARDÍACA, NA VARIABILIDADE

DA FREQÜÊNCIA CARDÍACA E NA

FREQÜÊNCIA RESPIRATÓRIA

Silvia Martins Meireles

SÃO PAULO

2005 


\title{
ESTUDO DOS EFEITOS DA PRÁTICA DE IOGA NA FREQÜÊNCIA CARDÍACA, NA VARIABILIDADE DA FREQÜÊNCIA CARDÍACA E NA FREQÜEENCIA RESPIRATÓRIA
}

\author{
SILVIA MARTINS MEIRELES
}

Dissertação apresentada à Escola de Educação Física e Esporte da Universidade de São Paulo, como requisito parcial para obtenção do grau de Mestre em Educação Física. 


\section{AGRADECIMENTOS}

A todos que são inspiradores de idéias e ideais...

À minha irmã - através da qual eu conheci a prática da ioga, ao Maurício L. Ferreira, que apontou para mim este caminho e à Irene G. Arcuri, que proporcionou o meu início como professora de ioga. Aos alunos, que me ajudam a trilhar e realizar esse caminho.

A todos os meus professores, pela formação acadêmica.

À professora Ignez N. Romeu, pelo respeito pela ioga e pela lição que deixou de sua vida. (Com sua determinação, foi a primeira mulher a participar em 1966-1967 do curso de ioga em Kaivalyadhama, Lonavla, Índia, até então exclusivo para homens. Tradutora do livro ASANAS, sendo a primeira edição em português datada de 1976 e professora de ioga na Escola de Educação Física da Universidade de São Paulo a partir de 1974). Ao Marcos R. Rodrigues, aluno da professora Ignez e meu professor, pelo incentivo e colaboração de sempre. Aos Swamis Kuvalayananda, Digambarji, e ao professor Gharote (in memorian), ao Swami Maheshananda, professores Pratap, Ganguly, Bhole, Gore, Alicia Souto e tantos outros pela companhia e acolhida em Lonavla. Aos colegas e amigos indianos e suas famílias, que foram todos meus professores em lições diárias de cultura indiana no tempo que estive na Índia.

Ao professor Alberto C. Amadio e à professora Idágene A. Cestari, que tornaram possível e realizável este antigo projeto de pesquisar ioga cientificamente. 
À Dra. Idágene, pela amizade e pelo tempo e interesse dedicados a este trabalho.

Ao Dr. Amadio pelos ensinamentos e pelo exemplo de respeito, amizade e atenção para com todos.

Ao Dr. Mauro R. Ushizima, pelo apoio e contribuições ao longo do desenvolvimento deste trabalho, pelo entusiasmo e dedicação pelo conhecimento e pela ciência e sua disponibilidade em ouvir e ensinar.

Ao Dr. Luis Mochizuki pela participação e sugestões no trabalho e pelo interesse em pesquisar e ensinar.

Aos colegas do laboratório de biomecânica da EEFE e dos laboratórios de engenharia de tecidos e de eletrônica do InCor pela amizade e dedicação que têm em seus trabalhos, pelo respeito e atenção para com as pessoas e competência em seus trabalhos.

Aos funcionários da secretaria de pós-graduação e da biblioteca da EEFE da USP pela gentileza de seus atendimentos.

A todos os voluntários pela disposição em participarem da pesquisa.

Ao meu pai Osvaldo F. Meirelles (in memorian) e à minha mãe, Feliciana M. Meireles, pelo exemplo e pelo que fazem por mim. À Selma e à Silvana, pelo que são, pelas lutas, reflexões e decisões que tomamos juntas.

À vida, que possibilita todos esses encontros.

Dedico este trabalho à professora Mestra Ignez Novaes Romeu (in memorian), pelo exemplo de seu caráter e de sua vida, dedicada a ensinar a ioga autêntica. 
LISTA DE TABELAS..........................................................

LISTA DE FIGURAS........................................................... ix

LISTA DE SIGLAS, ABREVIAÇÕES E SÍMBOLOS.................... xiii

LISTA DE ANEXOS............................................................

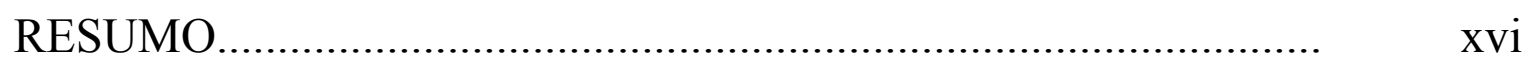

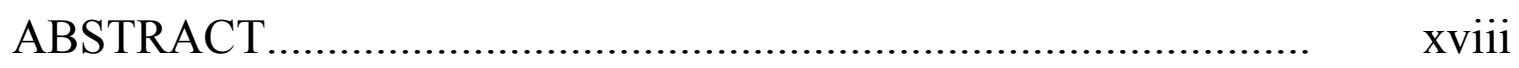

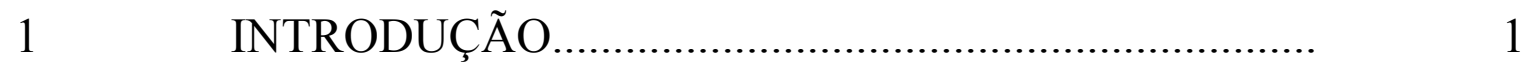

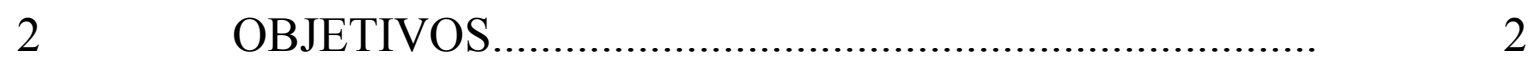

3 REVISÃO DA LITERATURA...................................... 2

3.1 A prática da ioga...................................................... 2

3.2 Ioga e Sistema Cardiovascular..........................................

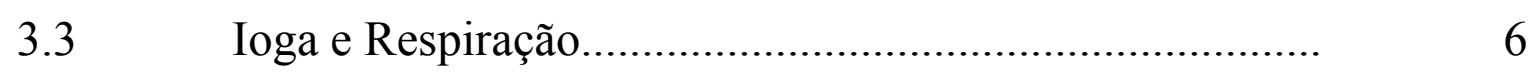

3.4 Análise espectral aplicada ao estudo dos efeitos da prática de ioga.............................................................. 9

4 MATERIAIS E MÉTODOS......................................... 12

4.1 Protocolo experimental............................................... 12

4.1.1 Registro dos sinais cárdio-respiratórios........................... 15

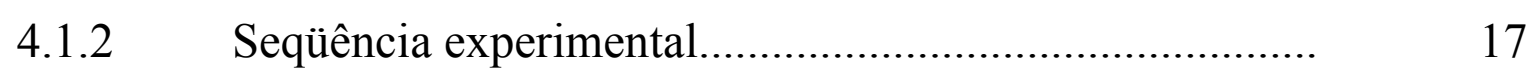

4.2 Análise dos sinais....................................................... 20

4.2.1 Pré-processamento do eletrocardiograma......................... 20

4.2.2 Pré-processamento do respirograma............................... 21

4.3 Análise espectral....................................................... 22 
4.4 Análise estatística....................................................... 23

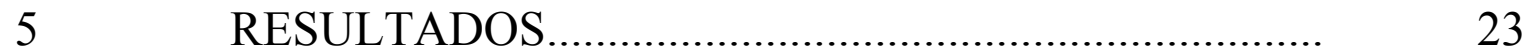

5.1 Análise no domínio do tempo para os grupo IOGA e

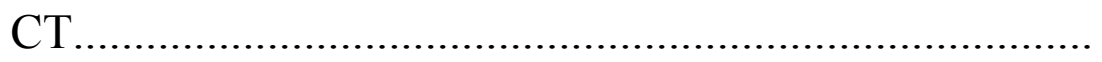

5.2 Análise no domínio da freqüência para os grupos IOGA

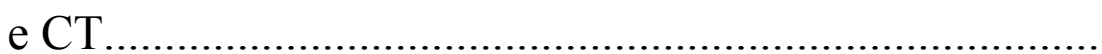

5.2.1 Alterações nas flutuações rítmicas dos sinais do

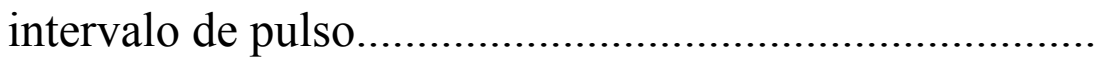

5.3 Análise no domínio do tempo para os grupos IOGAativ e CTativ.....

5.4 Análise no domínio da freqüência para os grupos IOGAativ e CTativ....................................................... 39

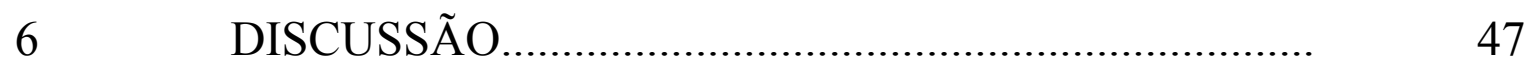

REFERÊNCIAS................................................................... 52

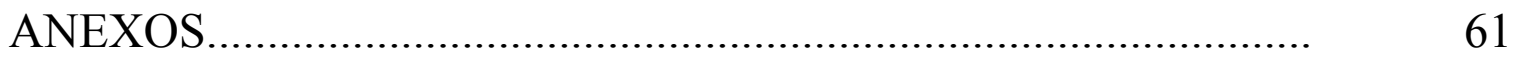




\section{LISTA DE TABELAS}

Página

TABELA 1 Principais estudos mostrando o efeito da prática de ioga sobre o sistema cardiorespiratório............

TABELA 2 Estudos sobre o efeito da prática de ioga no sistema cardiorespiratório e análise espectral.........................................................

TABELA 3 Características dos praticantes de ioga.................

TABELA 4

Características dos participantes do grupo controle.

TABELA 5

Atividades e freqüência de práticas físicas dos participantes dos grupos IOGA e CT e tempo e freqüência de prática de ioga dos participantes do grupo IOGA.

TABELA 6 Valores individuais e média (média $\pm \mathrm{dp}$ ) da FC, DFC, IP, DIP, FR e DFR no grupo IOGA na condição BASAL

TABELA 7 Valores individuais e média (média $\pm \mathrm{dp}$ ) da FC, DFC, IP, DIP, FR e DFR no grupo IOGA na condição APÓS A PRÁTICA..........................

TABELA 8 Valores individuais e média (média $\pm \mathrm{dp}$ ) da FC, DFC, IP, DIP, FR e DFR no grupo CT na condição BASAL................................................... 
TABELA 9 Valores individuais e média (média $\pm \mathrm{dp}$ ) da FC, DFC, IP, DIP, FR e DFR no grupo CT na condição APÓS A PRÁTICA...............................

TABELA 10 Valores individuais e média (média $\pm \mathrm{dp}$ ) da potência espectral do IP nas faixas $\mathrm{MBF}, \mathrm{BF}$, AF, PTOT e VAR do grupo IOGA na condição BASAL

TABELA 11 Valores individuais e média (média $\pm \mathrm{dp}$ ) da potência espectral do IP nas faixas MBF, BF, AF, PTOT e VAR do grupo CT na condição BASAL

TABELA 12 Valores individuais e média (média $\pm \mathrm{dp}$ ) da $\mathrm{BFn}, \mathrm{AFn}$ e BF/AF no grupo IOGA na condição BASAL

TABELA 13 Valores individuais e média (média $\pm \mathrm{dp}$ ) da $\mathrm{BFn}, \mathrm{AFn}$ e BF/AF no grupo $\mathrm{CT}$ na condição BASAL

TABELA 14 Valores individuais e média (média $\pm \mathrm{dp}$ ) MBF, BF e AF, PTOT e VAR no grupo IOGA na condição APÓS A PRÁTICA................................

TABELA 15 Valores individuais e média (média $\pm \mathrm{dp}$ ) da potência espectral do IP nas faixas MBF, BF e AF, PTOT e VAR no grupo CT na condição APÓS A PRÁTICA.

TABELA 16 Valores individuais e média (média $\pm \mathrm{dp}$ ) da $\mathrm{BFn}, \mathrm{AFn}$ e BF/AF no grupo IOGA na condição APÓS A PRÁTICA. 
TABELA 17 Valores individuais e média (média $\pm \mathrm{dp}$ ) da $\mathrm{BFn}, \mathrm{AFn}, \mathrm{BF} / \mathrm{AF}$ no grupo $\mathrm{CT}$ na condição APÓS A PRÁTICA...........................................

TABELA 18 Valores individuais e média (média $\pm \mathrm{dp}$ ) da MBF, BF e AF, PTOT e VAR no grupo IOGAativ na condição BASAL............................

TABELA 19 Valores individuais e média (média \pm dp) da MBF, BF e AF, PTOT e VAR no grupo CTativ na condição BASAL...........................................

TABELA 20 Valores individuais e média (média $\pm \mathrm{dp}$ ) da $\mathrm{BFn}, \mathrm{AFn}$ e BF/AF do grupo IOGAativ na condição BASAL

TABELA 21 Valores individuais e média (média $\pm \mathrm{dp}$ ) da BFn, AFn e BF/AF no grupo CTativ na condição BASAL....................................................

TABELA 22 Valores individuais e média (média $\pm \mathrm{dp}$ ) da $\mathrm{MBF}, \mathrm{BF}, \mathrm{AF}$, PTOT e VAR no grupo IOGAativ na condição APÓS A PRÁTICA........

TABELA 23 Valores individuais e média (média $\pm \mathrm{dp}$ ) da MBF, BF, AF, PTOT e VAR no grupo CTativ na condição APÓS A PRÁTICA

TABELA $24 \quad$ Valores individuais e média (média $\pm \mathrm{dp}$ ) da $\mathrm{BFn}, \mathrm{AFn}$ e BF/AF no grupo IOGAativ na condição APÓS A PRÁTICA................................

TABELA 25 Valores individuais e média (média $\pm \mathrm{dp}$ ) da $\mathrm{BFn}, \mathrm{AFn}$ e $\mathrm{BF} / \mathrm{AF}$ no grupo CTativ na condição APÓS A PRÁTICA.............................. 
TABELA $26 \quad$ Resumo dos resultados........................................ 46 


\section{LISTA DE FIGURAS}

Página

FIGURA 1 Faixas de flutuação do sinal de freqüência cardíaca. Faixas de alta freqüência (AF), de baixa freqüência $(B F)$ e de muito baixa freqüência (MBF). As barras ilustram os limites em freqüência e respectiva duração das flutuações de cada faixa

FIGURA 2 Fluxograma do protocolo experimental....................

FIGURA 3 Posição dos eletrodos e da cinta respiratória para registro do ECG e do sinal respiratório.

FIGURA 4

Segmento do sinal de ECG registrado.

FIGURA 5

Segmento do sinal da respiração registrado.

FIGURA 6

Seqüência experimental e duração das condições de registro do ECG e do sinal da respiração

FIGURA 7 Seqüência e descrição das posturas de ioga, número de repetições e tempo de prática estimado, utilizado no protocolo experimental........................

FIGURA 8 Registro de segmento do sinal de ECG original (A) e após a identificação da onda $\mathrm{R}$ em cada batimento (B)

FIGURA 9 Seqüência para obtenção do IP a partir do sinal de ECG. 
FIGURA 10 Segmento típico do sinal de respiração original (A) e após filtragem e identificação dos picos (B).........

FIGURA 11 Freqüência cardíaca (FC) nos grupos praticantes de ioga (IOGA) e controle (CT) nas condições BASAL e APÓS A PRÁTICA * $p<0,05 \ldots \ldots \ldots \ldots . . . . .$.

FIGURA 12 Intervalo de pulso (IP) no grupo praticantes de ioga (IOGA) e controle (CT) nas condições BASAL e APÓS A PRÁTICA * $\mathrm{p}<0,05 \ldots \ldots \ldots \ldots \ldots . . . . .$.

FIGURA 13 Desvio padrão do intervalo de pulso (DIP) nos grupos praticantes de ioga (IOGA) e controle (CT) nas condições BASAL e APÓS A PRÁTICA * $p<$ 0,05

FIGURA 14 Séries temporais de freqüência cardíaca (FC, bpm), freqüência respiratória (FR, cpm) e intervalo de pulso (IP, ms) .................................................... 28

FIGURA 15 Espectro de potência $\left(\mathrm{ms}^{2} / \mathrm{Hz}\right)$ do intervalo de pulso nas faixas estudadas $(\mathrm{Hz})$ obtido de em um praticante de ioga na condição BASAL (A) e APÓS A PRÁTICA de ioga (B)..............................

FIGURA 16 Potência total (PTOT) nos grupos IOGA e CT nas condições BASAL e APÓS A PRÁTICA * p < 0,05

FIGURA 17 Variância (VAR) nos grupos IOGA e CT nas condições BASAL e APÓS A PRÁTICA * p < 0,05 . 
FIGURA 18 Freqüência cardíaca (FC) nos grupos IOGAativ e 36 CTativ nas condições BASAL e APÓS A PRÁTICA * $\mathrm{p}<0,05$.

FIGURA 19 Desvio padrão da freqüência cardíaca (DFC) nos grupos IOGAativ e CTativ nas condições BASAL e APÓS A PRÁTICA * p $<0,05$

FIGURA 20 Intervalo de pulso (IP) nos grupos IOGAativ e CTativ nas condições BASAL e APÓS A PRÁTICA * $\mathrm{p}<0,05$ 38

FIGURA 21 Desvio do intervalo de pulso (DIP) nos grupos IOGAativ e CTativ nas condições BASAL e APÓS A PRÁTICA * $\mathrm{p}<0,05$ 38

FIGURA 22 Potência espectral na faixa de muito baixa freqüência (MBF) no grupo IOGAativ e CTativ nas condições BASAL e APÓS A PRÁTICA * p < 0,05

FIGURA 23 Potência espectral na faixa de baixa freqüência (BF) nos grupos IOGAativ CTativ, nas condições BASAL e APÓS A PRÁTICA * $\mathrm{p}<0,05$

FIGURA 24 Potência espectral na faixa de alta freqüência (AF) nos grupos IOGAativ CTativ, nas condições BASAL e APÓS A PRÁTICA * p < 0,05

FIGURA 25 Potência total (PTOT) do intervalo de pulso nos grupos IOGAativ CTativ nas condições BASAL e APÓS A PRÁTICA............................................. 
FIGURA 26 Variância (VAR) da potência total nos grupos IOGAativ e CTativ nas condições BASAL e

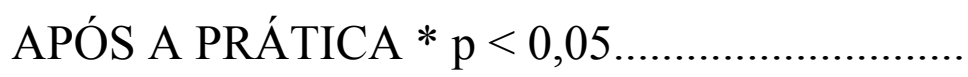




\section{LISTA DE SIGLAS, ABREVIAÇÕES E SÍMBOLOS}

A/D Conversor analógico-digital

AF Potência espectral na faixa de alta freqüência

AFn AF normalizada

BF Potência espectral na faixa de baixa freqüência

$\mathrm{BF} / \mathrm{AF} \quad$ Razão entre baixa freqüência e alta freqüência

BFn BF normalizada

bpm Batimentos por minuto

cpm Ciclos por minuto

CT Controle

DEP_IP Densidade espectral de potência do intervalo de pulso

DFC Desvio padrão da freqüência cardíaca

DFR Desvio padrão da freqüência respiratória

DIP Desvio padrão do intervalo de pulso

dp Desvio padrão

ECG Eletrocardiograma

FC Freqüência cardíaca

FR Freqüência respiratória

$\mathrm{kg} \quad$ Kilograma

$\mathrm{Hz} \quad$ Hertz

IP Intervalo de pulso cardíaco

IMC Índice de massa corpórea

m média

MBF Potência espectral na faixa de muito baixa freqüência 
ms Milisegundos

PTOT Potência total

S Segundos

VAR Variância 


\section{LISTA DE ANEXOS}

Página

ANEXO I

Questionário

para

registro

de

voluntários

61

ANEXO II

Termo de

consentimento

livre

e

esclarecido

64

ANEXO III Transcrição da gravação que antecede os registros.

ANEXO IV Resultados individuais. Séries temporais, DEP e DEP IP.

ANEXO V

Resultados da análise estatística entre os grupos

IOGA e CT.

ANEXO VI

Resultados da análise estatística entre os grupos IOGAativ e CTativ 


\title{
RESUMO
}

\section{ESTUDO DOS EFEITOS DA PRÁTICA DE IOGA NA FREQÜÊNCIA CARDÍACA, NA VARIABILIDADE DA FREQÜÊNCIA CARDÍACA E NA FREQÜÊNCIA RESPIRATÓRIA}

\author{
AUTORA: SILVIA MARTINS MEIRELES \\ ORIENTADORA: PROFA. DRA. IDÁGENE A. CESTARI
}

O objetivo deste estudo é avaliar os efeitos da prática da ioga na freqüência cardíaca, na variabilidade da freqüência cardíaca e na freqüência respiratória. Foram estudados praticantes de ioga (IOGA, $n=8)$ e não praticantes controles $(\mathrm{CT}, \mathrm{n}=8)$ com idade entre 30 e 45 anos. Dois sub-grupos de quatro indivíduos cada, com atividade física regular foram também comparados $\left(\right.$ IOGA $_{\text {ativ }}$ e $\mathrm{CT}_{\text {ativ }}$ ). Os participantes realizaram prática de asanas (IOGA) ou simulação (CT) precedidos e sucedidos por relaxamento, sendo registrados (20 min) o eletrocardiograma e respirograma antes e após os asanas ou simulação. A freqüência cardíaca foi menor no grupo IOGA comparado ao CT $(58,9 \pm 6,6$ e $64,3 \pm 5,6 \mathrm{bpm})$ e no grupo IOGA $_{\text {ativ }}$ comparado ao $\mathrm{CT}_{\text {ativ }}(55,0 \pm 3,6$ e 63,2 \pm $5,5 \mathrm{bpm})$. Não foram encontradas diferenças na freqüência respiratória. $\mathrm{O}$ intervalo de pulso (IP) foi maior no grupo IOGA (1036,0 $\pm 111,2 \mathrm{~ms})$ comparado ao $\mathrm{CT}(943,9 \pm 85,3 \mathrm{~ms})$ e IOGA $_{\text {ativ }}(1101,6 \pm 72,6 \mathrm{~ms})$ comparado ao $\mathrm{CT}_{\text {ativ }}$ 
(957,8 \pm 92,6 ms). Também o desvio padrão do IP foi maior no grupo IOGA $(68,8 \pm 22,8 \mathrm{~ms})$ comparado ao CT $(51,1 \pm 21,7 \mathrm{~ms})$ e grupo IOGA $_{\text {ativ }}(77,8 \pm$ $14,1 \mathrm{~ms})$ comparado ao $\mathrm{CT}_{\text {ativ }}(41,5 \pm 17,8 \mathrm{~ms})$. A potência espectral do IP foi maior no grupo IOGA ativ comparada ao $\mathrm{CT}_{\text {ativ }}$ na faixa de muito baixa freqüência $\left(2157,3 \pm 1159,4\right.$ vs. $\left.876,0 \pm 989,4 \mathrm{~ms}^{2}\right)$ e maior nas faixas de baixa $(1523,7 \pm$ 964,6 vs. $\left.494,7 \pm 417,1 \mathrm{~ms}^{2}\right)$ e alta freqüências $(1684,4 \pm 847,9$ vs. $462,6 \pm 375,1$ $\mathrm{ms}^{2}$ ) comparando-se os valores obtidos após os asanas ou simulação. Os resultados sugerem que a combinação de exercícios físicos e ioga pode aumentar os benefícios destes à saúde.

PALAVRAS-CHAVE: ioga, asanas, relaxamento, variabilidade da freqüência cardíaca, análise espectral, freqüência respiratória. 


\begin{abstract}
EFFECTS OF YOGA PRACTICE IN HEART RATE, HEART RATE VARIABILITY AND RESPIRATORY RATE
\end{abstract}

\author{
AUTHOR: SILVIA MARTINS MEIRELES \\ ADVISER: PROFA. DRA. IDÁGENE A. CESTARI
}

The objective of this work is to evaluate the effects of yoga practice in heart rate (HR), HR variability and respiratory rate using spectral analysis. We compared yoga students (IOGA, $\mathrm{n}=8)$ with non-practicing controls $(\mathrm{CT}, \mathrm{n}=8)$, between 30 and 45 years of age. These groups were subdivided in two subgroups $(n=4)$ having regular practice of physical activities besides yoga $\left(\mathrm{IOGA}_{\text {activ }}\right.$ and $\mathrm{CT}_{\text {activ }}$ ). The participants practiced the yoga asanas (IOGA) or physical simulation (CT) preceded and succeeded by relaxation, after which the electrocardiogram and respirogram was recorded for $20 \mathrm{~min}$. Heart rate was lower in IOGA when compared to CT $(58,9 \pm 6,6$ vs. $64,3 \pm 5,6 \mathrm{bpm})$ and in IOGA $_{\text {activ }}$ compared to $\mathrm{CT}_{\text {activ }}(55,0 \pm 3,6$ vs. $63,2 \pm 5,5 \mathrm{bpm})$. No diferences in respiratory rate were found between groups. Pulse interval (PI) was higher in IOGA when compared to CT $(1036,0 \pm 111,2$ vs. $943,9 \pm 85,3 \mathrm{~ms})$ and $\mathrm{IOGA}_{\text {activ }}$ compared to $\mathrm{CT}_{\text {activ }}(1101,6 \pm 72,6$ vs. $957,8 \pm 92,6 \mathrm{~ms})$. Standard deviation of pulse interval was greater in IOGA when compared to CT $(68,8 \pm 22,8$ vs. $51,1 \pm$ 
$21,7 \mathrm{~ms})$ and IOGA $_{\text {activ }}$ compared to $\mathrm{CT}_{\text {activ }}(77,8 \pm 14,1$ vs. $41,5 \pm 17,8 \mathrm{~ms})$. The spectral power of the PI was higher in the IOGA $_{\text {activ }}$ group in relation to the $\mathrm{CT}_{\text {activ }}$ in the bands of very low $\left(2157,3 \pm 1159,4\right.$ vs. $\left.876,0 \pm 989,4 \mathrm{~ms}^{2}\right)$ and in the band of low $\left(1523,7 \pm 964,6\right.$ vs. $\left.494,7 \pm 417,1 \mathrm{~ms}^{2}\right)$ and high frequency $\left(1684,4 \pm 847,9\right.$ vs. $\left.462,6 \pm 375,1 \mathrm{~ms}^{2}\right)$ after the practice of yoga asanas. These results suggest that combination of physical exercises and yoga may increase their healthy benefits.

KEYWORDS: yoga, asanas, relaxation, heart rate variability, spectral analysis, respiratory rate. 


\section{INTRODUÇÃO}

A ioga é um sistema filosófico indiano eminentemente prático que inclui práticas físicas corporais (asanas) e respiratórias (pranayamas). A palavra ioga, também escrita como yoga, deriva da raiz sânscrita "yuj" que significa unir, ligar ou controlar ELIADE (1954).

O termo ioga também é utilizado como integração ou "Samadhi” dos aspectos moral, social, físico, mental e espiritual do ser humano em oposição a "Vyadhi”, ou desintegração (KUVALAYANANDA \& VINEKAR, 1971).

A mais antiga compilação do sistema de ioga é atribuída a Patanjali em seu texto clássico "Yoga Sutras" (TAIMNI, 1996). A ioga se manteve como tradição oral e não existe registro preciso sobre a data do texto de Patanjali, mas presume-se que tenha sido elaborado entre os séculos II a.C. e II d.C. Estima-se, a partir de evidências arqueológicas, que a origem da ioga remonte a 2500 a.C. (KARAMBELKAR, 1986).

Efeitos benéficos para o corpo e a mente são comumente atribuídos à prática da ioga. Segundo textos clássicos da ioga, sua prática contribui para a manutenção ou recuperação do "equilíbrio" do corpo e da mente entendendo-se ainda que este equilíbrio é fundamental para o bem estar do ser humano (KUVALAYANANDA \& VINEKAR, 1971).

Os estudos científicos considerando os efeitos das práticas de ioga tiveram início na década de 1920, na Índia, com a primeira de uma série de publicações científicas intitulada "X-Ray experiments on Uddiyana and Nauli" e "Barometric Experiments on Nauli" em revista trimestral do Instituto Kaivalyadhama.

A ioga tem-se popularizado ao longo do tempo como uma prática de relaxamento para combater o estresse da sociedade moderna. No entanto, a 
popularização da prática não foi acompanhada de estudos científicos que pudessem identificar os fundamentos dos benefícios atribuídos à prática da ioga.

\section{OBJETIVOS}

Este trabalho tem como objetivo identificar e avaliar os efeitos da prática de ioga sobre os sistemas cardiovascular e respiratório. Os objetivos específicos deste trabalho compreendem:

a) estudar a freqüência cardíaca, a variabilidade da freqüência cardíaca e a freqüência respiratória em praticantes e não praticantes de ioga;

b) estudar a freqüência cardíaca, a variabilidade da freqüência cardíaca e a freqüência respiratória antes e após uma prática de ioga constituída por uma seqüência de asanas, em indivíduos praticantes de ioga, ou movimentos que simulam as alterações posturais realizadas durante os asanas, nos participantes não praticantes de ioga.

3.1 A prática da ioga

O texto elaborado por Patanjali, o Yoga Sutras, em forma de aforismos, é bastante conciso e nele estão expostas as normas que devem orientar a conduta do praticante (yamas e niyamas), a prática de posturas (asanas), as práticas de controle respiratório (pranayamas), o domínio gradual dos sentidos (pratiahara), o aperfeiçoamento e aprofundamento da atenção (dharana), da concentração (dhiana) e finalmente meditação (samadhi). Dois outros textos clássicos posteriores (Hathapradipika e Gheranda Samhita) escritos provavelmente entre os séculos XIV e XVIII descrevem, com detalhes, as técnicas enumeradas por Patanjali (DGAMBARJI \& GHAROTE, 1970, 
1978). Asana, também traduzido como postura, é um dos oito componentes do sistema de ioga de Patanjali. A palavra asana provém da raiz sânscrita "as" e significa sentar, embora o termo asana se aplique a posturas da ioga nas quais o praticante assume posição deitada ou também em pé. As posturas da ioga podem ser classificadas como posturas invertidas (nas quais o coração está em um nível acima da cabeça), posturas realizadas a partir da flexão, extensão, rotação e flexão lateral da coluna vertebral e posturas de equilíbrio. Os asanas são relativamente conhecidos pela semelhança com os exercícios físicos convencionais e têm como objetivo propiciar condições de saúde física e mental ao praticante. Patanjali descreve asana como uma postura associada à sensação de conforto e estabilidade na qual o praticante permanece sem a menor tendência para mover qualquer parte do corpo. De acordo com Patanjali, asana é essencialmente uma postura meditativa e não um exercício executado com objetivos somente físicos (DGAMBARJI \& SAHAY, 1991).

Textos posteriores aos de Patanjali descrevem a execução dos diversos asanas. Svatmarama em seu texto Hathapradipika relaciona 15 asanas e afirma que estes produzem estabilidade física e mental, saúde e sensação de leveza (DGAMBARJI \& GHAROTE, 1970). Gheranda, em seu texto Gheranda Samhita, define asana como aquele que "...fortalece o corpo..." e cita 32 asanas (DGAMBARJI \& GHAROTE, 1978).

Durante a prática, o padrão postural envolvido em cada asana é mais importante que a execução exata do movimento, uma vez que o esforço além da capacidade individual pode gerar expectativa, estresse mental e movimentos bruscos indesejáveis na prática da ioga KUVALAYANANDA e VINEKAR (1971). A execução do asana deve ser lenta, suave e contínua e a posição final é mantida estática por tempo gradualmente aumentado pelo praticante, de acordo com seus limites, a fim de atender às características de estabilidade e conforto. 
Agindo dessa forma, o limite físico bem como o tempo de permanência ideal passam a ser reconhecidos pelo praticante que aprende a respeitar e observar tais limites que são naturalmente alterados ao longo do tempo de prática.

GOPAL, ANANTHARAMN, NISHITH e BHATNAGAR (1974) salientam que "em ioga, durante a execução do asana, há um treino físico combinado ao relaxamento psicológico" e, portanto, de acordo com UDUPA, SINGH e SETTIWAR (1975) uma condição de "estabilidade neurofisiológica" é atingida.

\subsection{Ioga e Sistema Cardiovascular}

Os primeiros estudos envolvendo o sistema cardiovascular foram realizados por ANAND e CHHINA (1961) e WENGER, BAGCHI e ANAND (1961). Estes autores observaram aumento da pressão intratorácica em praticantes de ioga durante bloqueio da respiração após uma inspiração ou expiração profundas, com oclusão da glote e contração vigorosa dos músculos do tórax e abdome. Os autores concluíram que não houve controle voluntário sobre a atividade cardíaca, com a freqüência cardíaca mantida regular e havendo diminuição do débito cardíaco por diminuição do retorno venoso para o coração devido ao aumento da pressão intratorácica. Durante pausa respiratória em um iogue, sem encontrar alterações que sugerissem controle voluntário da atividade cardíaca, BHOLE e KARAMBELKAR (1972) assim como WENGER, BAGCHI e ANAND (1961) observaram aumento do intervalo entre pulsos cardíacos.

Outros estudos relataram diminuição de pressão arterial em pacientes hipertensos com a prática de relaxamento em postura de ioga (savasana) (DATEY, DESHMUK, DALVI \& VINEKAR, 1969; PATEL, 1973, 1975; 
PATEL \& NORTH, 1975; SANTAELLA, 2003; SUNDAR, AGRAWAL, SINGH, BHATTACHARYA, UDUPA \& VAISH, 1984).

A prática prolongada de ioga também foi estudada como prevenção e tratamento de doença arterial coronariana com diminuição de colesterol de baixa densidade, triglicérides, massa corporal e maior capacidade de realizar exercício físico, MANCHANDA e NARANG (1998) e diminuição do risco cardiovascular, DAMODARAN, MALATHI, PATIL, SHAH, SURYAVANSHI e MARATHE (2002) constatada pela diminuição das freqüências cardíaca $(76,4$ $\pm 1,6$ vs. $70,4 \pm 2,2 \mathrm{bpm})$ e respiratória (18,2 $\pm 1,08$ vs. $15,2 \pm 1,62 \mathrm{cpm})$ e da pressão arterial (sistólica e diastólica), diminuição do nível de catecolaminas na urina e aumento de colinesterase, após três meses de uma hora diária de prática de ioga.

BERNARDI, SPADACINI, BELLWON, HAJRIC, ROSKAMM e FREY (1998) observaram efeitos benéficos da respiração lenta e controlada em pacientes com insuficiência cardíaca crônica, tais como aumento na saturação de oxigênio e capacidade para o exercício e sugerem que o controle respiratório, também presente na prática de ioga, pode ser aplicado junto a outras formas de tratamento destes pacientes.

$\mathrm{O}$ efeito agudo da prática de relaxamento em postura da ioga (savasana) foi estudada por SANTAELLA, $2003 \mathrm{em}$ indivíduos normotensos e hipertensos e neste estudo foi observada diminuição da freqüência cardíaca em ambos os grupos.

BOWMAN, CLAYTON, MURRAY, REED, SUBHAN e FORD (1997) estudaram o intervalo R-R em idosos sedentários distribuídos de maneira a formar dois grupos, que realizaram seis semanas de exercício aeróbico em bicicleta ou prática de ioga constituída de asanas, pranayamas e relaxamento. Houve diminuição da FC (69 \pm 8 vs. $\left.61 \pm 7 \mathrm{~min}^{-1}\right)$. As alterações em parâmetros 
bioquímicos e endócrinos resultantes da prática prolongada de ioga mostraram aumento da atividade adrenocortical sugerindo maior capacidade de superar estresses segundo estudos de UDUPA e SINGH (1972) e UDUPA, SINGH e YADAV (1973). Outros estudos utilizando diferentes asanas sugerem que cada asana tem uma influência endócrina e metabólica específica (UDUPA, SINGH \& SETTIWAR, 1975). Além de parâmetros bioquímicos, JOSEPH, SRIDHARAN, PATIL, KUMARIA, SELVAMURTHY, JOSEPH e NAYAR (1981) estudaram parâmetros fisiológicos antes e após três meses de prática diária de uma hora de ioga, composta por asanas, pranayamas e meditação. Os autores observaram juntamente com alterações bioquímicas, redução na freqüência cardíaca (64 $\pm 1,90$ para $59 \pm 2,30 \mathrm{bpm}$ ), na pressão arterial sistólica $(117 \pm 1,60$ para $114 \pm 1,80 \mathrm{~mm} \mathrm{Hg})$ e diastólica $(80 \pm 1,30$ para $73 \pm 1,60 \mathrm{~mm}$ Hg) e sugerem alteração no balanço autonômico com predomínio parassimpático.

\subsection{Ioga e Respiração}

O sistema respiratório recebeu atenção especial dos antigos iogues como uma forma de "treino" do sistema nervoso uma vez que a respiração é uma função que apresenta os controles voluntário e involuntário. Também, "a mucosa olfatória representa a localização anatômica onde o sistema nervoso está mais próximo ao meio externo", GANONG (1993); SOVIK, (2000). A alternância de congestão e descongestão da mucosa nasal, denominado como ciclo nasal (FLANAGAN \& ECCLES, 1997) também foi explorada pelos iogues havendo diversos tipos de pranayamas nos quais a respiração é realizada através de uma narina por vez.

O controle voluntário sobre a atividade respiratória foi investigado em praticantes de ioga por ANAND, CHHINA e SINGH (1961). Estes autores 
observaram as respostas respiratória e metabólica à alta concentração de $\mathrm{CO}_{2} \mathrm{em}$ um iogue que permaneceu durante dez horas deitado no interior de uma câmara com volume de ar conhecido, medindo-se o consumo de $\mathrm{O}_{2}$ e a concentração de $\mathrm{CO}_{2}$ no gás expirado. Simultaneamente, o eletroencefalograma, o eletrocardiograma e os movimentos respiratórios foram registrados. Neste experimento observou-se que o iogue não apresentava hiperpnéia ou taquicardia mesmo quando havia diminuição de oxigênio e aumento na concentração de dióxido de carbono havendo diminuição do consumo de $\mathrm{O}_{2}$ e eliminação de $\mathrm{CO}_{2}$ muito abaixo dos valores basais. Posteriormente, STANESCU, NEMERY, VERITER e MARECHAL (1981) estudaram a resposta ventilatória ao $\mathrm{CO}_{2} \mathrm{em}$ praticantes e não praticantes de ioga durante respiração com duração das fases controlada e observaram que devido à respiração lenta, ambos os grupos apresentavam respostas menores àquela condição. Quando realizados os testes com respiração em ar com maior concentração de $\mathrm{CO}_{2}$ verificou que os dois grupos de indivíduos aumentavam a ventilação ao final do teste, mas que os praticantes de ioga apresentavam, nos dois últimos minutos do teste, freqüência respiratória menor daquelas encontradas no grupo controle e atribuem essas características à prática repetida de exercícios respiratórios da ioga. Os autores verificaram que os praticantes de ioga apresentavam maior volume corrente e respiração mais lenta quando comparados aos não praticantes durante respirações livres. Dentre as explicações sugeridas está uma diminuída atividade simpática nos iogues.

SPICUZZA, GABUTTI, PORTA, MONTANO e BERNARDI (2000) investigaram se a sensibilidade reflexa dos quimiorreceptores era alterada pela respiração lenta ou devido à prática de ioga e concluíram que a respiração lenta reduzia a sensibilidade quimiorreflexa, mas que a prática prolongada de ioga resultava na redução generalizada da atividade reflexa dos quimiorreceptores. 
BERNARDI, PASSINO, WILMERDING, DALLAM, PARKER, ROBERT e APPENZELLER (2001), a fim de observarem o efeito da respiração lenta para obter aumento de oxigenação e redução dos efeitos da ativação simpática proveniente da exposição à altitude, compararam um grupo de praticantes de ioga a um grupo controle em uma condição de altitude simulada e observaram que a respiração dos praticantes de ioga foi mais eficiente do que a respiração lenta executada pelo grupo controle, havendo menor diminuição na saturação do oxigênio no grupo de praticantes de ioga.

Na TABELA 1 são apresentados os principais resultados encontrados na literatura dos efeitos da prática de ioga no sistema cardiorespiratório.

TABELA 1 - Principais estudos mostrando o efeito da prática de ioga sobre o sistema cardiorespiratório.

\begin{tabular}{|c|c|c|c|c|c|c|c|}
\hline Autor & Tempo de prática & $\mathrm{FC}$ & DFC & IP & DIP & FR & DFR \\
\hline $\begin{array}{l}\text { Udupa et al., } \\
1975\end{array}$ & $\begin{array}{l}24 \text { semanas de prática diária } \\
\text { (asanas) }\end{array}$ & NS & & & & NS & \\
\hline $\begin{array}{l}\text { Joseph et al., } \\
1981\end{array}$ & $\begin{array}{l}12 \text { semanas de prática diária com } \\
\text { duração de } 1 \text { hora (asanas, } \\
\text { pranayama e meditação). }\end{array}$ & $\downarrow$ & & & & & \\
\hline $\begin{array}{l}\text { Bowman et } \\
\text { al, } 1997\end{array}$ & $\begin{array}{l}6 \text { semanas de prática, } 2 \text { vezes por } \\
\text { semana e duração de } 90 \text { minutos. } \\
\text { (asanas, pranayamas) }\end{array}$ & $\downarrow$ & & & & & \\
\hline Leite, 1999 & 3 a 30 anos de prática de ioga & & & & & & \\
\hline $\begin{array}{l}\text { Bernardi et } \\
\text { al., } 2001\end{array}$ & $\begin{array}{l}24 \text { semanas a } 20 \text { anos (asanas e } \\
\text { pranayamas) }\end{array}$ & & & & & NS & \\
\hline $\begin{array}{l}\text { Damodaran } \\
\text { et al., } 2002\end{array}$ & $\begin{array}{l}12 \text { semanas de prática diárias com } \\
1 \text { hora de duração (asanas, } \\
\text { pranayamas) }\end{array}$ & $\downarrow$ & & & & $\downarrow$ & \\
\hline $\begin{array}{l}\text { Santaella, } \\
2003\end{array}$ & 20 minutos de prática (savasana) & $\downarrow$ & & & & & \\
\hline
\end{tabular}

A seta indica a modificação encontrada e NS indica que não houve diferença significante. $\mathrm{O}$ campo em branco indica que a variável não foi estudada. 
3.4 Análise espectral aplicada ao estudo dos efeitos da prática de ioga

A análise espectral permite decompor uma série temporal em suas componentes em freqüência e quantificar a amplitude relativa das mesmas.

Um dos estudos pioneiros que demonstram o uso da análise espectral foi realizado por AKSELROD, GORDON, UBEL, SHANNON, BARGER e COHEN em 1981, estudando as variações da freqüência cardíaca (FC) demonstraram a relação entre os sistemas de regulação e as flutuações na FC identificando faixas que sugeriam a regulação nervosa sobre o sistema cardiovascular.

Em humanos, as faixas de freqüência com relevância biológica sugeridas pela Sociedade Européia de Cardiologia (TASK FORCE OF THE EUROPEAN SOCIETY OF CARDIOLOGY THE NORTH AMERICAN SOCIETY OF PACING ELECTROPHYSIOLOGY, 1996) correspondem à alta freqüência $(\mathrm{AF})$ entre 0,15 a $0,4 \mathrm{~Hz}$; à baixa freqüência $(\mathrm{BF})$ entre 0,04 a 0,15 $\mathrm{Hz}$ e muito baixa freqüência (MBF) com valores menores ou iguais a $0,04 \mathrm{~Hz}$. As potências para cada faixa de freqüência são obtidas a partir do cálculo da área sob a curva da densidade espectral de potência do intervalo de pulso nas respectivas faixas.

A faixa de $\mathrm{AF}$ corresponde à modulação parassimpática sobre o nó sinusal e também reflete a arritmia sinusal respiratória (variações rítmicas na freqüência cardíaca que ocorrem na mesma freqüência de ativação da respiração). A faixa de BF do espectro da variabilidade da freqüência cardíaca contém um maior componente simpático e também da modulação parassimpática e está associada à atividade vasomotora. A potência de muito baixa freqüência, observada em análises de registros longos (24 horas) do eletrocardiograma tem sido relacionada à termorregulação e flutuações hormonais (SLEIGHT \& CASADEI, 1995). 
A FIGURA 1 apresenta, de forma esquemática, as modulações encontradas nos sinais do sistema cardiovascular atribuídas às atividades respiratória, vasomotora, hormonal e termoreguladora com as faixas de freqüências e períodos respectivos.

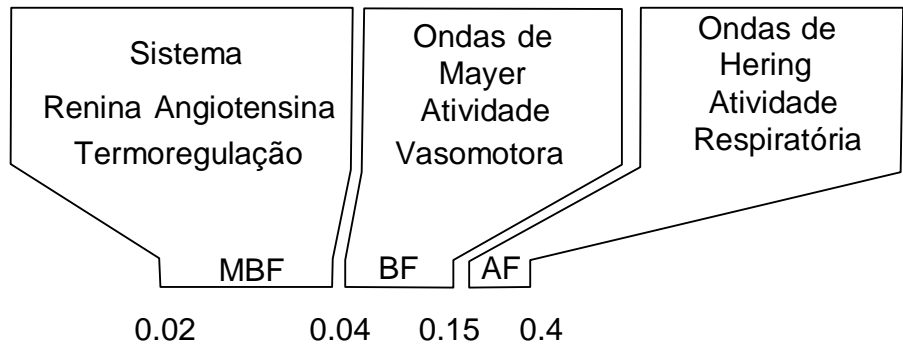

Freqüência

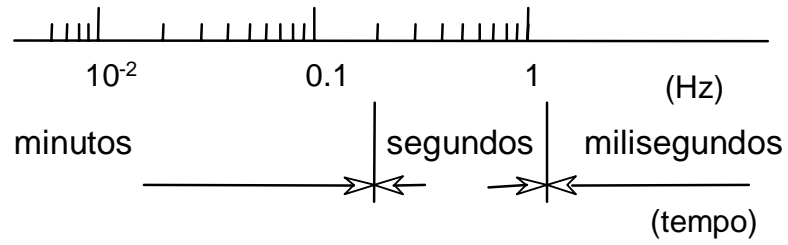

FIGURA 1 - Faixas de flutuação do sinal de freqüência cardíaca. Faixas de alta freqüência $(\mathrm{AF})$, de baixa freqüência $(\mathrm{BF})$ e de muito baixa freqüência (MBF). As barras ilustram os limites em freqüência e respectiva duração das flutuações de cada faixa.

Em 1986, PAGANI, LOMBARDI, GUZZETTI, RIMOLDI, FURLAN, PIZZINELLI, SANDRONE, MALFATTTO, DELL'ORTO, PICCALUGA, TURIEL, BASELLI, CERUTTI e MALLIANI propuseram que o cálculo da razão da potência espectral da faixa de baixa freqüência e de alta freqüência $(\mathrm{BF} / \mathrm{AF})$ representa a interação, ou equilíbrio simpato-vagal. A hipótese do balanço simpato-vagal encontra restrições em condições experimentais tais como exercício físico intenso ou insuficiência cardíaca severa MALLIANI, LOMBARDI e PAGANI (1994) e ainda em freqüências respiratórias baixas, as quais se somam à faixa de baixa freqüência do espectro de potência (BROWN, BEIGHTOL, KOH \& ECKBERG, 1993). 
SANTAELLA (2003), usando análise espectral, comparou os efeitos da prática de exercício físico, de relaxamento em postura da ioga (savasana) e de exercíco físico combinado ao relaxamento em indivíduos normotensos e hipertensos observando, em ambos os grupos, efeito hipotensor maior quando estas condutas foram combinadas.

Os efeitos fisiológicos de diferentes técnicas de meditação que utilizavam ritmos respiratórios distintos foram estudados por PENG, HENRY, MIETUS, HAUSDORFF, KHALSA, BENSON e GOLDBERGER (2004) através da análise espectral do intervalo RR do eletrocardiograma durante a prática. Houve aumento na potência de baixa freqüência $(\mathrm{BF})$ nas práticas de meditação nas quais a freqüência respiratória média foi de seis a oito ciclos por minuto (cpm) e aumento da freqüência cardíaca. Da mesma forma, LEAL (1996) estudou diferentes manobras respiratórias nas quais praticantes de ioga realizavam inspiração lenta seguida de pausa respiratória e de expiração lenta ou respirações rápidas seguidas de pausa respiratória e de expiração lenta observando durante a execução destas, aumento da freqüência cardíaca em ambos os tipos de respiração. Houve diminuição da potência total devido à respiração rápida (140 ciclos por minuto) e aumento na variância do intervalo R$\mathrm{R}$ e aumento na potência de muito baixa freqüência nas respirações lentas, atribuindo estas respostas à ressonância respiratória devido à freqüência respiratória ser de um a dois ciclos por minuto.

LEHRER, SASAKI e SAITO (1999) estudaram a variabilidade cardíaca durante a meditação Zazen e observaram que a amplitude total da variabilidade da freqüência cardíaca aumentou durante a prática de meditação. A respiração lenta provocou aumento na faixa de baixa freqüência do espectro. 
Na TABELA 2 são apresentados os estudos nos quais a análise espectral foi aplicada ao estudo dos efeitos da prática de ioga como proposto neste estudo.

TABELA 2 - Estudos sobre o efeito da prática de ioga no sistema cardiorespiratório e análise espectral.

\begin{tabular}{|c|c|c|c|c|c|c|c|c|c|}
\hline Autor & Tempo de prática & MBF & $\mathrm{BF}$ & $\mathrm{AF}$ & PTOT & VAR & $\mathrm{BFn}$ & AFn & $\mathrm{BF} / \mathrm{AF}$ \\
\hline $\begin{array}{l}\text { Bowman } \\
\text { et at, } \\
1997\end{array}$ & $\begin{array}{l}6 \text { semanas de } \\
\text { prática, } 2 \text { vezes por } \\
\text { semana, e duração } \\
\text { de } 90 \text { minutos. } \\
\text { (asanas, } \\
\text { pranayamas) }\end{array}$ & & $\uparrow$ & NS & & & & & \\
\hline
\end{tabular}

A seta indica a modificação encontrada e NS indica que não houve diferença significante. $\mathrm{O}$ campo em branco indica que a variável não foi estudada.

\section{MATERIAIS E MÉTODOS}

4.1 Protocolo experimental

Os participantes do estudo tem idade entre 30 e 45 anos, não possuem doenças crônicas ou fazem uso de medicamentos rotineiramente. Todos os participantes responderam um questionário informando os hábitos referentes à atividade física. Cópia deste questionário encontra-se no ANEXO I.

Os praticantes de ioga (IOGA), praticam ioga há no mínimo um ano, com regularidade de ao menos uma prática semanal com duração de 60 min. $\mathrm{O}$ grupo controle (CT) inclui não praticantes de ioga.

Oito indivíduos voluntários, praticantes de ioga, com idade entre 33 e 44 anos compõem o grupo de praticantes de ioga (IOGA) deste estudo. Algumas 
das características desses voluntários constam da TABELA 3, com valores individuais e média (média \pm dp).

TABELA 3 - Características dos praticantes de ioga.

\begin{tabular}{cccccc}
\hline IOGA & Sexo & $\begin{array}{c}\text { Idade } \\
(\text { ano })\end{array}$ & $\begin{array}{c}\text { Estatura } \\
(\mathrm{m})\end{array}$ & $\begin{array}{c}\text { Massa } \\
\text { corporal }(\mathrm{kg})\end{array}$ & $\begin{array}{c}\mathrm{IMC} \\
\left(\mathrm{kg} / \mathrm{m}^{2}\right)\end{array}$ \\
\hline 1 & $\mathrm{~F}$ & 34 & 1,64 & 54 & 20,08 \\
2 & $\mathrm{~F}$ & 35 & 1,55 & 52 & 21,64 \\
3 & $\mathrm{~F}$ & 39 & 1,72 & 63 & 21,30 \\
4 & $\mathrm{~F}$ & 44 & 1,67 & 59 & 21,16 \\
5 & $\mathrm{~F}$ & 40 & 1,62 & 55 & 20,96 \\
6 & $\mathrm{~F}$ & 35 & 1,72 & 69 & 23,32 \\
7 & $\mathrm{M}$ & 33 & 1,70 & 58 & 20,07 \\
8 & $\mathrm{~F}$ & 33 & 1,60 & 48 & 18,75 \\
média \pm dp & & $36,6 \pm 4,0$ & $1,65 \pm 0,06$ & $57,3 \pm 6,6$ & $20,9 \pm 1,3$ \\
\hline
\end{tabular}

Oito indivíduos voluntários, com idade entre 31 e 43 anos compõem o grupo controle (CT) deste estudo. Algumas das características do grupo controle constam da TABELA 4, com valores individuais e média (média \pm dp).

TABELA 4 - Características dos participantes do grupo controle

\begin{tabular}{cccccc}
\hline CT & Sexo & $\begin{array}{c}\text { Idade } \\
(\text { ano })\end{array}$ & $\begin{array}{c}\text { Estatura } \\
(\mathrm{m})\end{array}$ & $\begin{array}{c}\text { Massa } \\
\text { corporal }(\mathrm{kg})\end{array}$ & $\begin{array}{c}\mathrm{IMC} \\
\left(\mathrm{kg} / \mathrm{m}^{2}\right)\end{array}$ \\
\hline 1 & $\mathrm{~F}$ & 35 & 1,70 & 58 & 20,07 \\
2 & $\mathrm{~F}$ & 42 & 1,55 & 63 & 26,22 \\
3 & $\mathrm{~F}$ & 42 & 1,62 & 60 & 22,86 \\
4 & $\mathrm{~F}$ & 43 & 1,56 & 57 & 23,42 \\
5 & $\mathrm{~F}$ & 41 & 1,43 & 48 & 23,47 \\
6 & $\mathrm{M}$ & 36 & 1,80 & 90 & 27,78 \\
7 & $\mathrm{~F}$ & 37 & 1,54 & 56 & 23,61 \\
8 & $\mathrm{M}$ & 31 & 1,70 & 65 & 22,49 \\
média $\pm \mathrm{dp}$ & & $38,4 \pm 4,3$ & $1,61 \pm 0,12$ & $62,1 \pm 12,4$ & $23,7 \pm 2,3$ \\
\hline
\end{tabular}

As atividades físicas realizadas pelos indivíduos de ambos os grupos, informadas no questionário, estão relacionadas na TABELA 5. 
TABELA 5 - Atividades e freqüência de práticas físicas dos participantes dos grupos IOGA e CT e tempo e freqüência de prática de ioga dos participantes do grupo IOGA.

\begin{tabular}{|c|c|c|c|c|c|c|c|}
\hline & Atividades Físicas & 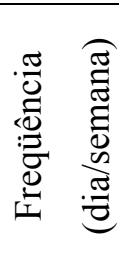 & 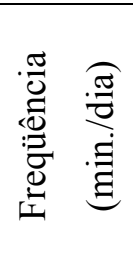 & 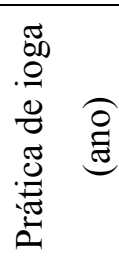 & 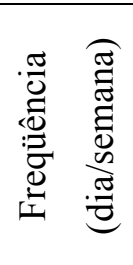 & 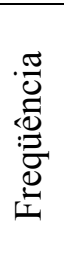 & 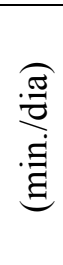 \\
\hline IOGA 1 & Natação & 2 & 30 & 4 & 3 & & 30 \\
\hline IOGA 2 & Corrida, natação e dança & 3 & 60 & 9 & 6 & & 30 \\
\hline IOGA 3 & Natação & 2 & 60 & 15 & 4 & & 60 \\
\hline IOGA 4 & $\begin{array}{c}\text { Caminhada, musculação, } \\
\text { hidroginástica }\end{array}$ & 4 & 60 & 9 & 2 & & 60 \\
\hline IOGA 5 & -------- & --- & --- & 14 & 6 & & 30 \\
\hline IOGA 6 & Corrida & 3 & 90 & 1 & 1 & & 60 \\
\hline IOGA 7 & --------- & --- & --- & 8 & 6 & & 60 \\
\hline IOGA 8 & Surfe & 1 & 150 & 10 & 2 & & 60 \\
\hline CT 1 & Caminhada & 2 & 60 & --- & --- & & --- \\
\hline CT 2 & Caminhada & 2 & 20 & --- & --- & & --- \\
\hline CT 3 & Kung fu & 2 & 90 & --- & --- & & --- \\
\hline CT 4 & Tai chi-chuan & 1 & 60 & --- & --- & & --- \\
\hline CT 5 & --------- & --- & --- & --- & --- & & --- \\
\hline CT 6 & Futebol & 1 & 60 & --- & --- & & --- \\
\hline CT 7 & --------- & --- & --- & --- & --- & & --- \\
\hline CT 8 & Caminhada & 2 & 30 & --- & --- & & --- \\
\hline
\end{tabular}

Os sinais do eletrocardiograma e respirograma foram registrados nos participantes sempre entre oito e 14 horas. Os voluntários foram instruídos a permanecer durante as vinte e quatro horas anteriores ao estudo sem ingestão de álcool, café, chocolate, ou qualquer outro estimulante ou medicamento que pudesse provocar alteração na atividade cardíaca. O registro foi realizado no Laboratório de Biomecânica da Universidade de São Paulo. Durante as sessões de registro, a temperatura ambiente, medida com termômetro de mercúrio, foi de $19^{\circ} \mathrm{C}$ a $25^{\circ} \mathrm{C}$ e a iluminação normal do local, com lâmpadas fluorescentes foi mantida. A circulação de pessoas no local do experimento foi restrita, mas não impedida. 
Ao chegar ao laboratório, os participantes foram informados quanto aos procedimentos e concordaram, por escrito, a participar como voluntários do estudo, de acordo com o protocolo aprovado pelo comitê de ética da Escola de Educação Física da USP. Cópia deste termo encontra-se no ANEXO II. Colchonetes foram dispostos em uma área do laboratório onde não havia estímulos visuais (como painéis para leitura), foi mantido silêncio, mas nenhuma alteração foi feita para tornar o ambiente livre de ruídos sonoros provenientes de telefone, portas, carros na rua, passos, vozes, etc.

As etapas do protocolo experimental estão esquematizadas no fluxograma apresentado na FIGURA 2.

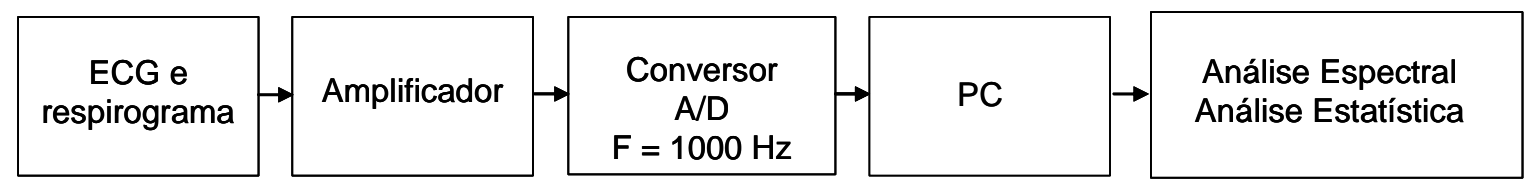

FIGURA 2 - Fluxograma do protocolo experimental.

As alterações nas freqüências cardíaca e respiratória antes e após a prática de ioga serão estudadas em ambos os grupos a partir do registro dos sinais de eletrocardiograma e respirograma.

4.1.1 Registro dos sinais cárdio-respiratórios

O eletrocardiograma (ECG) é obtido através de eletrodos de superfície (Meditrace) conectados a um amplificador isolador (Isolated Preamplifier, Gould Model 13-5407-58, USA) utilizando a derivação III do eletrocardiógrafo (ECG/Biotach, Gould, USA). O sinal elétrico na saída do eletrocardiógrafo é amplificado e filtrado entre 0,05 e $30 \mathrm{~Hz}$ (Amplificador Universal. Gould, USA). Após filtragem, o sinal é digitalizado com freqüência de amostragem de $1000 \mathrm{~Hz}$ (Windaq DI 220, Dataq Instruments, USA), para incluir os 
componentes relevantes do sinal original (ROMPELMMAN, SNIJDERS \& SPRONSEN, 1982).

O registro do sinal respiratório é feito simultaneamente ao registro de ECG utilizando-se uma cinta respiratória (Pneumotrace II, Modelo 1132, Respiration Transducer, UFI, USA) posicionada no tórax. O sinal proveniente da cinta respiratória é amplificado e filtrado entre $0,05 \mathrm{~Hz}$ e $30 \mathrm{~Hz}$ (Amplificador Universal, Gould, USA) e digitalizado com freqüência de amostragem de $1000 \mathrm{~Hz}$ (Windaq, Dataq Instruments, USA).

A FIGURA 3 ilustra esquematicamente a posição dos eletrodos de ECG e da cinta respiratória.

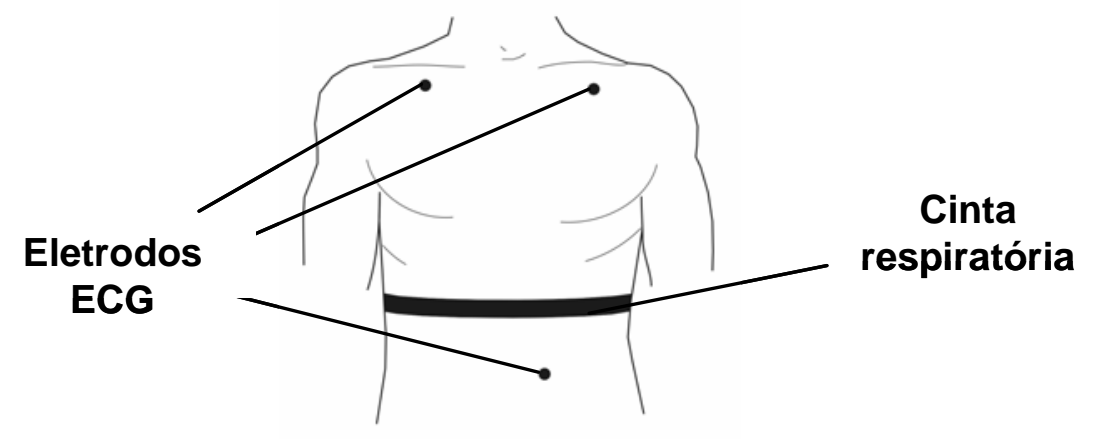

FIGURA 3 - Posição dos eletrodos e da cinta respiratória para registro do ECG e do sinal respiratório.

A FIGURA 4 ilustra um segmento típico do sinal de ECG registrado em um dos participantes do protocolo.

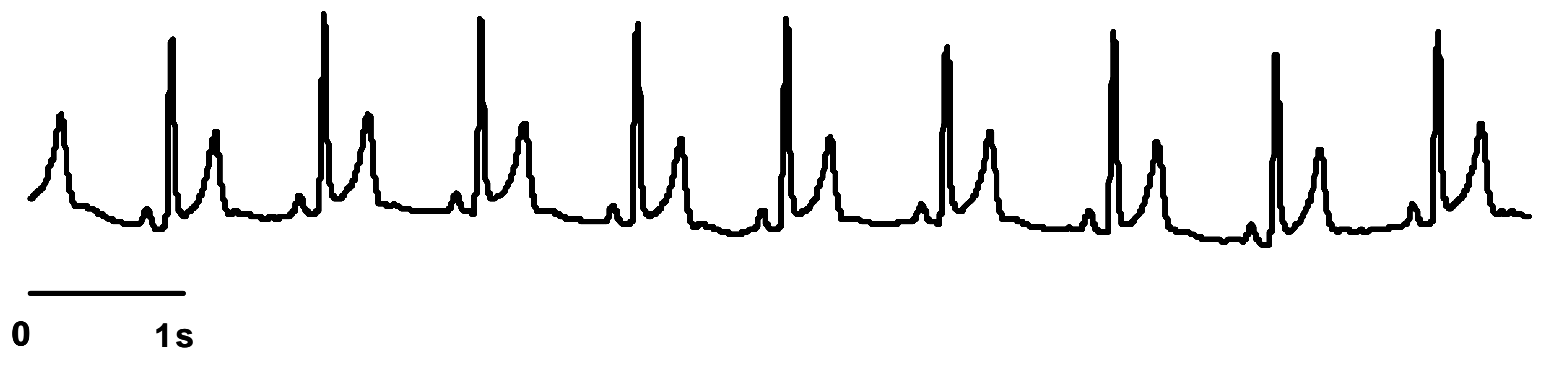

FIGURA 4 - Segmento do sinal de ECG registrado. 
A FIGURA 5 ilustra um segmento do sinal da respiração registrado em um dos praticantes do protocolo.

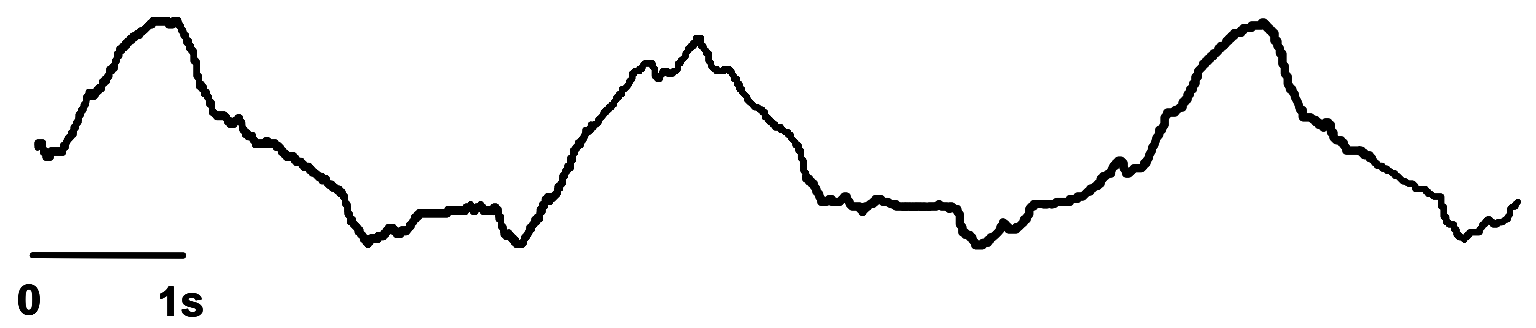

FIGURA 5 - $\underline{\text { Segmento do sinal da respiração registrado. }}$

\subsubsection{Seqüência experimental}

A seqüência experimental para registro dos sinais constitui-se de quatro condições: preparação, basal, prática e após a prática. Esta seqüência está representada na FIGURA 6.

A preparação para os registros consiste inicialmente na exposição do protocolo ao voluntário, preenchimento do questionário e assinatura do termo de participação livre e esclarecido pelo voluntário.

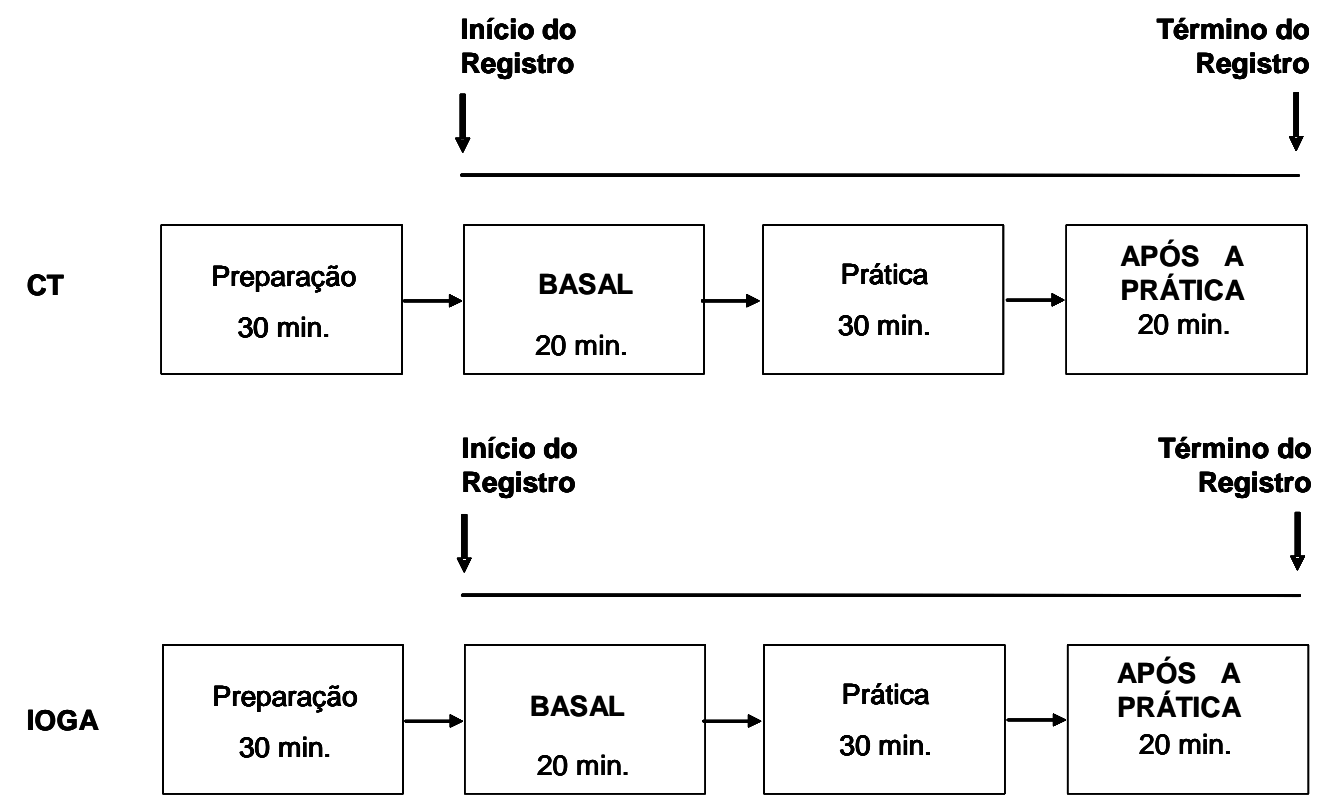

FIGURA 6 - Seqüência experimental e duração das condições de registro do ECG e do sinal da respiração. 
Após a apresentação e o preenchimento do questionário é feita a colocação dos eletrodos e da cinta respiratória. A seguir o participante permanece em decúbito dorsal a fim de adaptar-se ao ambiente, às condições do experimento e permitir que os parâmetros fisiológicos estejam próximos aos valores normais. Sempre que solicitado foi fornecido ao participante, almofadas para apoio da região lombar ou cervical e uma manta. A posição e atitude esperadas do participante, ou seja, uma posição confortável e manter-se acordado durante o tempo de registro, são informadas através de uma gravação (ANEXO III). Após a primeira parte da gravação, tem início o registro dos sinais do ECG e da respiração na condição basal durante 20 minutos (BASAL). Após o registo BASAL, o participante é orientado a sentar-se também pela gravação.

Em seguida os praticantes de ioga realizaram a seqüência de asanas conforme ilustrado na figura FIGURA 7. Os participantes do grupo controle realizaram a mesma seqüência de movimentos sugerida aos praticantes de ioga para simular as alterações posturais. As posturas foram apresentadas aos participantes no momento em que estes preenchiam o questionário para registro de voluntários.

A seqüência de asanas escolhida neste estudo consistiu de movimentos de extensão, flexões frontal e lateral e rotação da coluna vertebral, que pudessem ser realizados sem dificuldades pelos participantes não habituados com a prática de ioga. A forma de realização das posturas segue o sistema de ioga tradicional recomendada pelo Instituto Kaivalyadhama citada em KUVALAYANANDA (1993) e a seqüência proposta foi avaliada por um dos professores (Dr. Gharote) deste instituto (comunicação pessoal). 
1 - Brahma Mudra

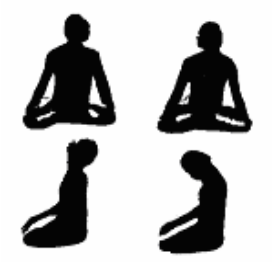

2 - Parvatasana

3 - Bhujangasana

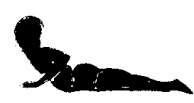

4 - Vakrasana

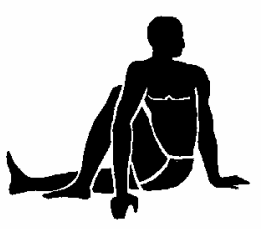

5 - Paschimatanasana

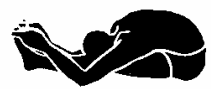

6 - Savasana

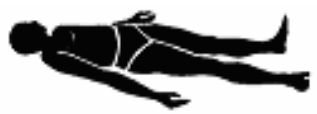

7 - Chakrasana

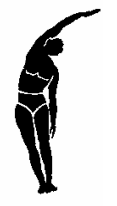

8 - Yoga Mudra

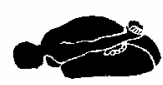

Sentado, pernas cruzadas, rotação para a direita, para a esquerda, extensão e flexão cervicais.

Número de repetições: 3

Tempo aproximado: $5 \mathrm{~min}$.

Sentado, pernas cruzadas, realizar extensão dos ombros.

Número de repetições: 2

Tempo aproximado: $3 \mathrm{~min}$.

Decúbito ventral, pernas unidas. Executar extensão da coluna cervical e torácica (sem extensão dos cotovelos).

Número de repetições: 2

Tempo aproximado: $4 \mathrm{~min}$.

Sentado, joelho direito estendido e joelho esquerdo flexionado com a planta do pé esquerdo apoiada no chão na altura do joelho direito. Executar a rotação lenta da coluna para o lado esquerdo, estando a mão esquerda apoiada no chão por trás do corpo e o braço direito apoiado externamente à coxa esquerda. Repetir o movimento no sentido contrário. Número de repetições: 2

Tempo aproximado: 5 min.

Sentado, pernas unidas, joelhos estendidos, realizar flexão do quadril.

Número de repetições: 2

Tempo aproximado: 4 min.

Decúbito dorsal, pernas e pés afastados entre si e braços naturalmente afastados do tronco.

Número de repetições: 1

Tempo aproximado: $1 \mathrm{~min}$.

Pés paralelos, extensão do ombro seguida de flexão contra lateral da coluna. Repetir o movimento no sentido contrário.

Número de repetições: 2

Tempo aproximado: 3 min.

Sentado, com joelhos flexionados, realizar flexão do quadril.

Número de repetições: 2

Tempo aproximado: 4 min.

FIGURA 7 - Seqüência e descrição das posturas de ioga, número de repetições e tempo de prática estimado, utilizado no protocolo experimental. 
Após a prática dos asanas ou prática de ioga simulada e estando o participante posicionado em decúbito dorsal, o registro dos sinais do ECG e da respiração foi realizado durante 20 min (APÓS A PRÁTICA).

\subsection{Análise dos sinais}

4.2.1 Pré-processamento do eletrocardiograma

Após a identificação dos eventos referentes à onda $\mathrm{R}$ do eletrocardiograma, os artefatos são identificados por inspeção visual e removidos manualmente em editor de sinais comercial (Windaq Waveform Browser, versão 2.10, Dataq Instruments, USA). A FIGURA 8-A ilustra um segmento de sinal de ECG e a figura 8-B ilustra o mesmo sinal com a onda $\mathrm{R}$ identificada em cada batimento cardíaco.

O intervalo de pulso (IP) é calculado pela diferença dos instantes de ocorrência entre picos consecutivos e preparado para a análise espectral conforme método padronizado por USHIZIMA (2000) que permite a análise por transformada rápida de Fourier. A FC é calculada (média e desvio padrão) por rotinas de um aplicativo comercial (MatLab, Mathwork Inc.)

A
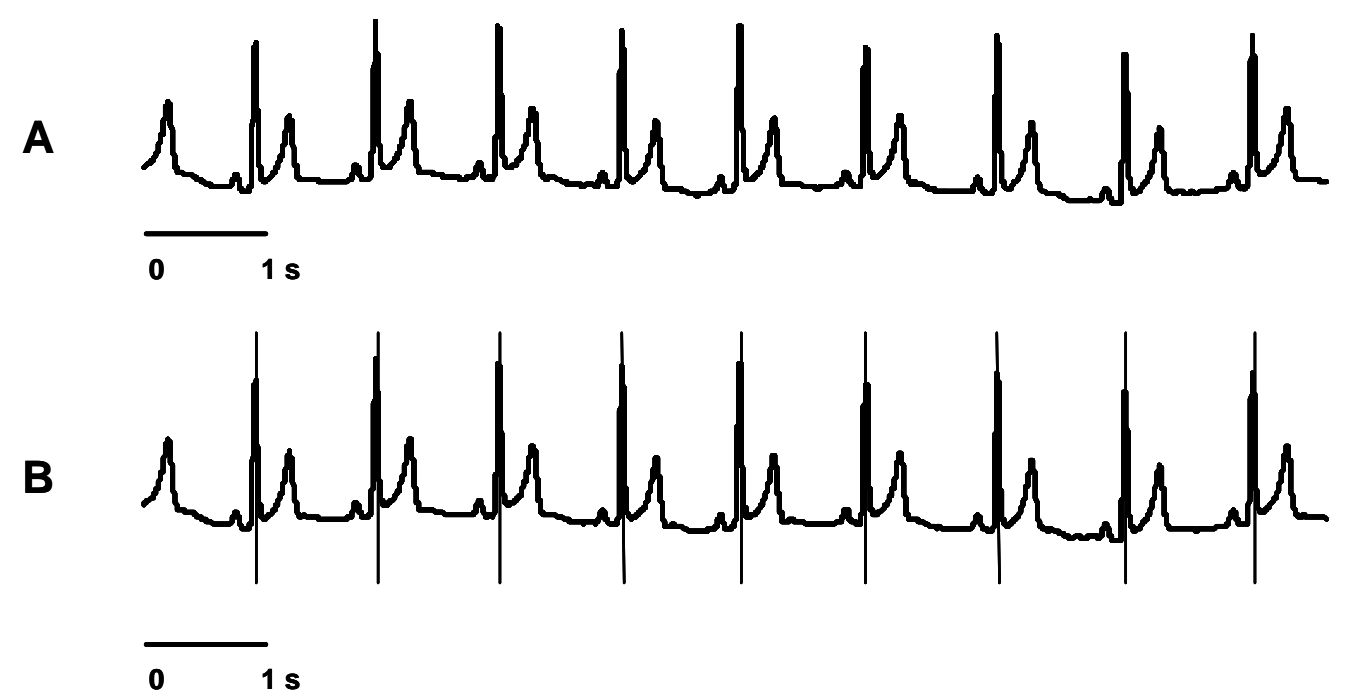

FIGURA 8 - $\underline{\text { Registro de segmento do sinal de ECG original (A) e após a }}$ identificação da onda $\mathrm{R}$ em cada batimento (B). 
A FIGURA 9 representa a seqüência de passos para obtenção do IP pelo processamento do sinal de ECG.

Sinal original

ECG<smiles>C1=CCC1</smiles>

Detecta pico<smiles>C1=CC=C1</smiles>

Cálculo do

Intervalo

$I P(n)=t_{n+1}-t_{n}$
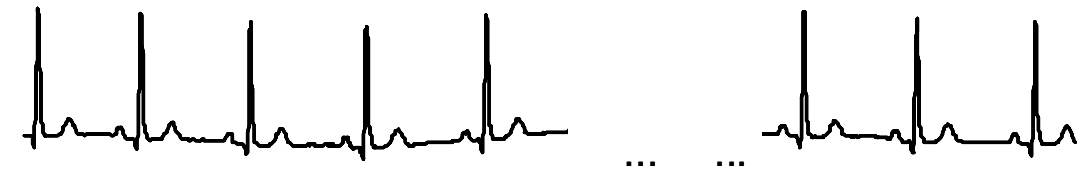

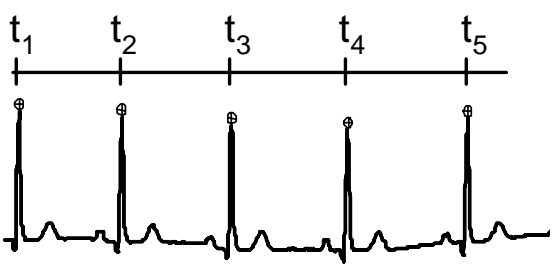

$\mathrm{IP}_{3} \quad \mathrm{IP}_{4}$

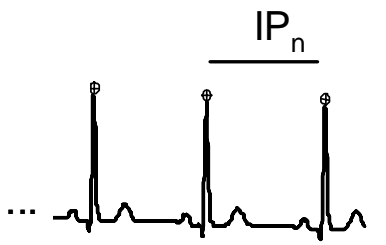

IP $\mathrm{n}$

FIGURA 9 - Seqüência para obtenção do IP a partir do sinal de ECG.

\subsubsection{Pré-processamento do respirograma.}

Para obtenção da freqüência respiratória (FR), o sinal original da respiração é filtrado digitalmente (Windaq- Dataq Instruments Calc Package Version 3.25) através da média móvel com 1000 pontos. A FIGURA 10 ilustra um segmento de sinal original de um respirograma (A) e o mesmo sinal após filtragem e identificação dos picos (B).

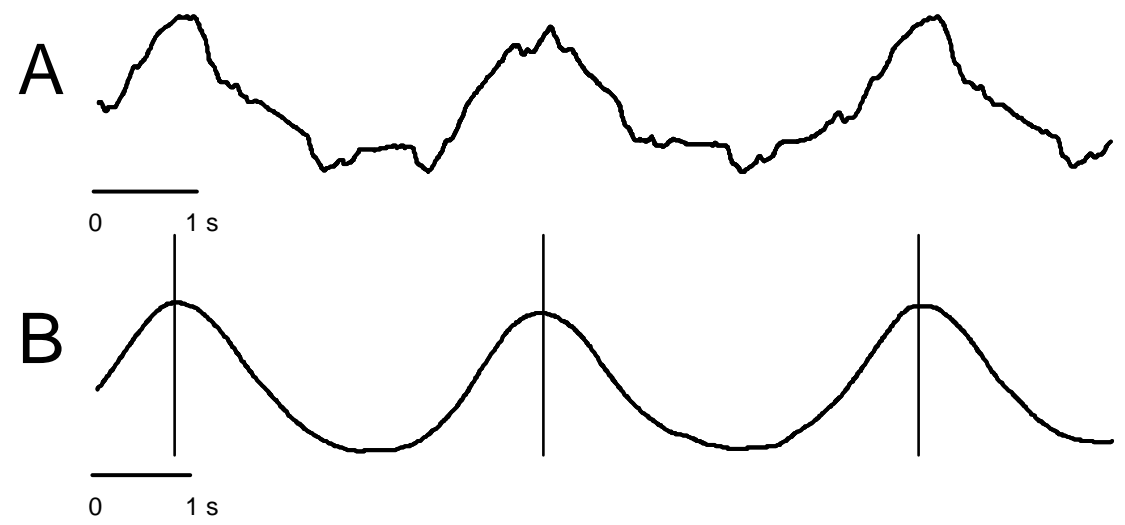

FIGURA 10 - Segmento típico do sinal de respiração original (A) e após filtragem e identificação dos picos (B). 
Os ciclos respiratórios são identificados através dos picos detectados e marcados automaticamente pelo aplicativo. Os artefatos são removidos após inspeção visual. A FR é calculada pelo inverso do intervalo entre picos consecutivos com rotinas de um aplicativo comercial (MatLab, Mathwork Inc.)

\subsection{Análise espectral}

Neste estudo, a densidade espectral de potência do IP (DEP_IP) é calculada pelo método Welch, com 1024 pontos, sobreposição de 50\%, janela Hanning, freqüência de re-amostragem de $13,9 \mathrm{~Hz}$ com uso da função pwelch do aplicativo Matlab (Mathwork Inc). A potência espectral é apresentada por seu valor absoluto $\left(\mathrm{ms}^{2}\right)$ e por unidade normalizada (n) conforme recomendado na literatura (TASK FORCE OF THE EUROPEAN SOCIETY OF CARDIOLOGY THE NORTH AMERICAN SOCIETY OF PACING ELECTTOPHYSIOLOGY, 1996).

O valor normalizado para cada faixa de freqüência é calculado pela equação abaixo (MALLIANI, LOMBARDI e PAGANI, 1994).

$$
P n=\frac{P}{\operatorname{var}-M B F} 100
$$

onde: Pn é a potência espectral normalizada (n) $\mathrm{P}$ é a potência espectral na faixa de interesse $\left(\mathrm{ms}^{2}\right)$ var é a variância do IP $\left(\mathrm{ms}^{2}\right)$

MBF é a potência espectral abaixo de $0,04 \mathrm{~Hz}\left(\mathrm{~ms}^{2}\right)$

A potência total (PTOT) é obtida a partir do cálculo da área sob a curva da DEP_IP. 
4.4 Análise estatística

As variáveis analisadas são: freqüência cardíaca (FC), desvio padrão da freqüência cardíaca (DFC), freqüência respiratória (FR), desvio padrão da freqüência respiratória (DFR), intervalo de pulso (IP), desvio padrão do intervalo de pulso (DIP), potência espectral na faixa de alta freqüência (AF), potência espectral na faixa de baixa freqüência $(\mathrm{BF})$, potência espectral na faixa de muito baixa freqüência (MBF), potência total (PTOT), variância (VAR), AF normalizada (AFn), BF normalizada (BFn), e razão entre BF e $\mathrm{AF}$ (BF/AF).

Foi utilizada análise de variância para dois fatores considerando-se como fator 1 os praticantes de ioga e não praticantes de ioga e fator 2 a condição anterior e após prática dos asanas. Foi adotado o valor de $\mathrm{p}<0,05$ como limite de significância para interpretação dos resultados e utilizado pós-teste de Bonferroni para comparações múltiplas. Para a análise estatística foi utilizado o programa SigmaStat (FOX, SHOTTON \& ULRICH, 1995). Para variáveis com distribuição não normal foi feita análise de variância não paramétrica utilizando teste de Friedman (Matlab Mathwork Inc.).

\section{RESULTADOS}

5.1 Análise no domínio do tempo para os grupo IOGA e CT

A seguir estão apresentados os valores encontrados para cada participante nas condições BASAL e APÓS A PRÁTICA, bem como o valor médio e desvio-padrão do grupo IOGA e CT.

Todas as variáveis passaram pelo teste de normalidade exceto $\mathrm{BF} / \mathrm{AF}$ e BF no grupo IOGA e CT e BF/AF no grupo IOGAativ e CTativ. 
A TABELA 6 e a TABELA 7 mostram os valores individuais das FC, DFC, IP, DIP, FR e DFR no grupo IOGA nas condições BASAL e APÓS A PRÁTICA.

TABELA 6 - Valores individuais e média (média \pm dp) da FC, DFC, IP, DIP, FR e DFR no grupo IOGA na condição BASAL.

\begin{tabular}{ccccccc}
\hline & $\begin{array}{c}\text { FC } \\
(\mathrm{bpm})\end{array}$ & $\begin{array}{c}\text { DFC } \\
(\mathrm{bpm})\end{array}$ & $\begin{array}{c}\text { IP } \\
(\mathrm{ms})\end{array}$ & $\begin{array}{c}\text { DIP } \\
(\mathrm{ms})\end{array}$ & $\begin{array}{c}\text { FR } \\
(\mathrm{cpm})\end{array}$ & $\begin{array}{c}\text { DFR } \\
(\mathrm{cpm})\end{array}$ \\
\hline 1 & 52,7 & 3,2 & 1142,7 & 69,8 & 11,6 & 2,3 \\
2 & 55,7 & 3,1 & 1080,5 & 58,9 & 17,8 & 1,8 \\
3 & 60,0 & 5,6 & 1008,5 & 85,3 & 18,3 & 3,3 \\
4 & 55,0 & 2,0 & 1091,4 & 39,9 & 9,7 & 3,0 \\
5 & 63,1 & 3,5 & 953,1 & 50,5 & 13,5 & 1,9 \\
6 & 57,5 & 4,4 & 1049,4 & 76,9 & 16,2 & 2,0 \\
7 & 70,7 & 3,7 & 851,2 & 44,8 & 15,8 & 1,8 \\
8 & 66,6 & 7,3 & 912,3 & 107,7 & 9,1 & 4,6 \\
média & 60,2 & 4,1 & 1011,1 & 66,7 & 14,0 & 2,6 \\
dp & 6,2 & 1,7 & 99,1 & 22,9 & 3,6 & 1,0 \\
\hline
\end{tabular}

TABELA 7 - Valores individuais e média (média \pm dp) da FC, DFC, IP, DIP, FR e DFR no grupo IOGA na condição APÓS A PRÁTICA.

\begin{tabular}{ccccccc}
\hline & $\begin{array}{c}\text { FC } \\
(\mathrm{bpm})\end{array}$ & $\begin{array}{c}\text { DFC } \\
(\mathrm{bpm})\end{array}$ & $\begin{array}{c}\text { IP } \\
(\mathrm{ms})\end{array}$ & $\begin{array}{c}\text { DIP } \\
(\mathrm{ms})\end{array}$ & $\begin{array}{c}\text { FR } \\
(\mathrm{cpm})\end{array}$ & $\begin{array}{c}\text { DFR } \\
(\mathrm{cpm})\end{array}$ \\
\hline 1 & 52,4 & 2,7 & 1148,9 & 59,2 & 11,3 & 2,4 \\
2 & 55,6 & 5,0 & 1086,9 & 94,8 & 11,9 & 4,7 \\
3 & 57,3 & 5,7 & 1056,6 & 93,3 & 17,2 & 3,5 \\
4 & 52,8 & 2,0 & 1138,3 & 42,7 & 14,1 & 3,0 \\
5 & 61,4 & 3,0 & 980,0 & 46,2 & 12,8 & 2,5 \\
6 & 48,7 & 3,6 & 1239,2 & 84,6 & 15,9 & 1,8 \\
7 & 72,0 & 4,1 & 836,1 & 48,0 & 17,7 & 2,4 \\
8 & 60,5 & 6,0 & 1001,5 & 98,3 & 11,4 & 4,5 \\
média & 57,6 & 4,0 & 1060,9 & 70,9 & 14,1 & 3,1 \\
dp & 7,2 & 1,4 & 123,5 & 24,1 & 2,6 & 1,0 \\
\hline
\end{tabular}


A TABELA 8 e a TABELA 9 mostram os valores individuais das FC, DFC, IP, DIP, FR e DFR no grupo CT nas condições BASAL e APÓS A PRÁTICA.

TABELA 8 - Valores individuais e média (média \pm dp) da FC, DFC, IP, DIP, FR e DFR no grupo CT na condição BASAL.

\begin{tabular}{ccccccc}
\hline & $\begin{array}{c}\text { FC } \\
(\mathrm{bpm})\end{array}$ & $\begin{array}{c}\text { DFC } \\
(\mathrm{bpm})\end{array}$ & $\begin{array}{c}\text { IP } \\
(\mathrm{ms})\end{array}$ & $\begin{array}{c}\text { DIP } \\
(\mathrm{ms})\end{array}$ & $\begin{array}{c}\text { FR } \\
(\mathrm{cpm})\end{array}$ & $\begin{array}{c}\text { DFR } \\
(\mathrm{cpm})\end{array}$ \\
\hline 1 & 60,2 & 4,0 & 1000,8 & 64,8 & 9,9 & 2,8 \\
2 & 67,1 & 2,3 & 894,7 & 30,2 & 13,4 & 2,4 \\
3 & 65,2 & 1,9 & 921,6 & 26,5 & 15,2 & 1,3 \\
4 & 56,8 & 3,0 & 1059,1 & 54,4 & 12,1 & 2,1 \\
5 & 73,3 & 5,7 & 823,5 & 65,6 & 15,2 & 2,0 \\
6 & 66,8 & 3,2 & 899,9 & 44,7 & 15,3 & 4,1 \\
7 & 73,5 & 3,9 & 818,1 & 43,0 & 15,8 & 1,8 \\
8 & 63,5 & 6,6 & 955,1 & 94,4 & 7,6 & 1,9 \\
média & 65,8 & 3,8 & 921,6 & 52,9 & 13,1 & 2,3 \\
dp & 5,8 & 1,6 & 82,7 & 22,1 & 3,0 & 0,8 \\
\hline
\end{tabular}

TABELA 9 - Valores individuais e média (média \pm dp) da FC, DFC, IP, DIP, FR e DFR no grupo CT na condição APÓS A PRÁTICA.

\begin{tabular}{ccccccc}
\hline & $\begin{array}{c}\text { FC } \\
(\mathrm{bpm})\end{array}$ & $\begin{array}{c}\text { DFC } \\
(\mathrm{bpm})\end{array}$ & $\begin{array}{c}\text { IP } \\
(\mathrm{ms})\end{array}$ & $\begin{array}{c}\text { IP } \\
(\mathrm{ms})\end{array}$ & $\begin{array}{c}\text { FR } \\
(\mathrm{cpm})\end{array}$ & $\begin{array}{c}\text { DFR } \\
(\mathrm{cpm})\end{array}$ \\
\hline 1 & 58,8 & 3,3 & 1023,0 & 57,4 & 10,5 & 2,4 \\
2 & 66,5 & 1,9 & 903,2 & 25,3 & 14,2 & 2,1 \\
3 & 62,9 & 1,9 & 954,2 & 28,1 & 15,3 & 1,7 \\
4 & 52,9 & 3,7 & 1140,4 & 76,3 & 12,5 & 2,4 \\
5 & 68,1 & 5,4 & 885,8 & 67,8 & 17,4 & 2,0 \\
6 & 67,7 & 3,4 & 889,0 & 46,2 & 15,2 & 3,3 \\
7 & 64,8 & 1,5 & 925,8 & 20,2 & 14,4 & 2,1 \\
8 & 59,9 & 4,6 & 1007,3 & 72,9 & 10,0 & 2,4 \\
média & 62,7 & 3,2 & 966,1 & 49,3 & 13,7 & 2,3 \\
dp & 5,2 & 1,4 & 87,4 & 22,6 & 2,5 & 0,5 \\
\hline
\end{tabular}


A FIGURA 11 ilustra os valores de freqüência cardíaca obtidos a partir do registro do ECG nos grupos IOGA e CT.

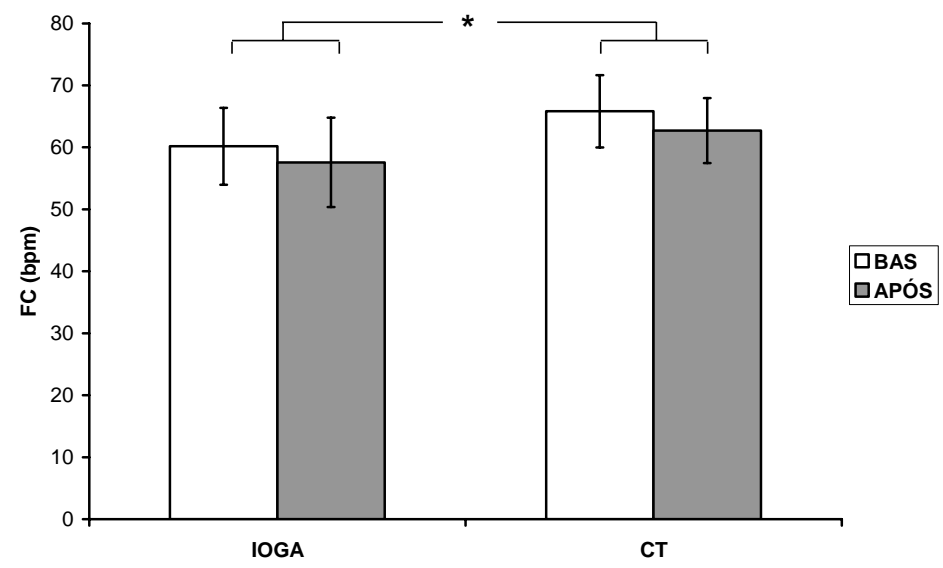

FIGURA 11 - Freqüência cardíaca (FC) nos grupos praticantes de ioga (IOGA) e controle (CT) nas condições BASAL e APÓS A PRÁTICA $* \mathrm{p}<0,05$.

Como observado na figura, a freqüência cardíaca não apresentou diferença para o mesmo grupo quando comparadas as condições BASAL e APÓS A PRÁTICA. No entanto, quando comparados os grupos, independentemente das fases BASAL ou APÓS A PRÁTICA, houve diferença significativa. A FC no grupo IOGA $(58,9 \pm 6,6 \mathrm{bpm})$ foi menor em relação ao grupo CT $(64,3 \pm 5,6 \mathrm{bpm}, \mathrm{p}=0,020)$.

O intervalo de pulso não apresentou diferença no mesmo grupo quando comparadas as condições BASAL e APÓS A PRÁTICA. Quando comparados os grupos, houve diferença significativa no IP, independentemente das fases BASAL ou APÓS A PRÁTICA. O IP no grupo IOGA $(1036,0 \pm 111,2$ ms) foi maior em relação ao IP no grupo CT $(943,9 \pm 85,3 \mathrm{~ms}, \mathrm{p}=0,014)$. 
A FIGURA 12 ilustra os valores obtidos para o intervalo de pulso (IP) nos grupos IOGA e CT.

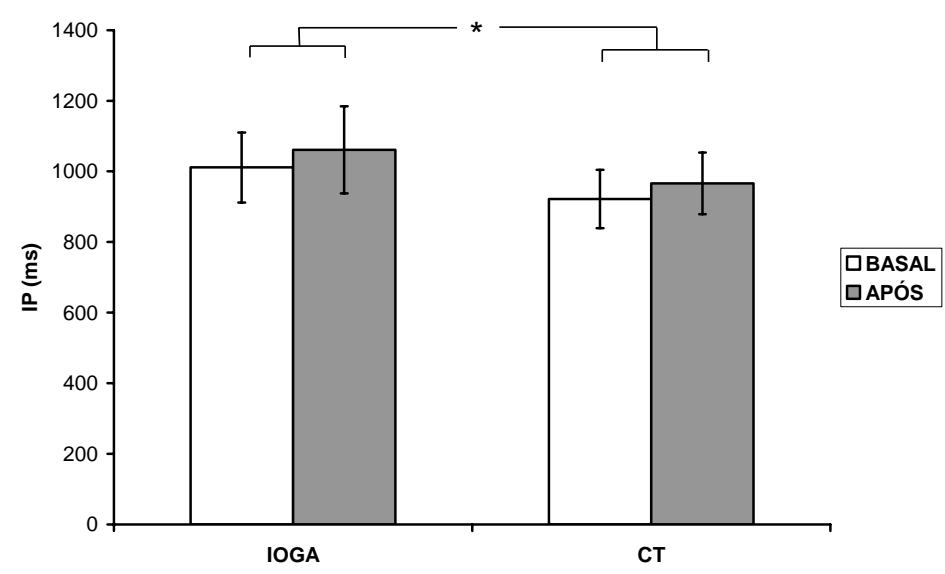

FIGURA 12 - Intervalo de pulso (IP) no grupo praticantes de ioga (IOGA) e controle (CT) nas condições BASAL e APÓS A PRÁTICA * $\mathrm{p}<0,05$

A FIGURA 13 ilustra o desvio padrão do intervalo de pulso (DIP) nos grupos IOGA e CT.

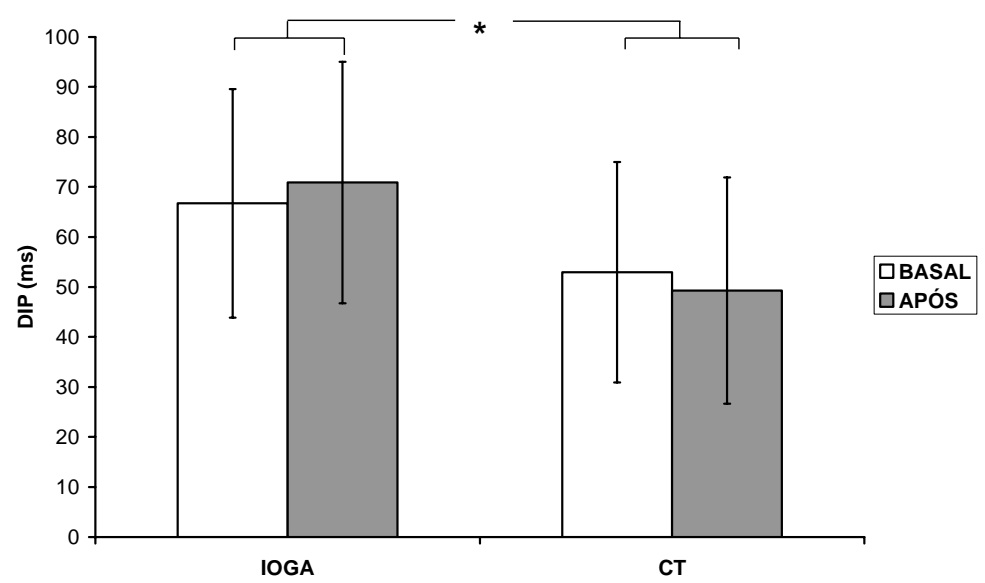

FIGURA 13 - Desvio padrão do intervalo de pulso (DIP) nos grupos praticantes de ioga (IOGA) e controle (CT) nas condições BASAL e APÓS A PRÁTICA * $p<0,05$.

Para um mesmo grupo, não houve diferença no desvio padrão do intervalo de pulso quando comparadas as condições BASAL e APÓS A 
PRÁTICA. No entanto, houve diferença significativa entre os grupos quando consideradas as condições BASAL e APÓS A PRÁTICA sem distinção. O valor do DIP no grupo IOGA foi maior $(68,8 \pm 22,8 \mathrm{~ms})$ em relação ao valor do DIP no grupo CT $(51,1 \pm 21,7 \mathrm{~ms}, \mathrm{p}=0,038)$.

Não houve diferença significativa quando comparados os grupos IOGA e CT para os valores obtidos da FR $(14,0 \pm 3,0 \mathrm{cpm}$ e 13,4 $\pm 2,7 \mathrm{cpm} ; \mathrm{p}=$ $0,542)$, do DFR $(2,8 \pm 1,0 \mathrm{cpm}$ e $2,3 \pm 0,7 \mathrm{cpm} ; \mathrm{p}=0,088)$ e do DFC $(4,0 \pm 1,5$ bpm e 3,5 $\pm 1,5$ bpm; $\mathrm{p}=0,334)$.

5.2 Análise no domínio da freqüência para os grupos IOGA e CT

A FIGURA 14 ilustra como exemplo, as séries temporais dos valores de FC, FR e IP obtidas do registro de um participante.
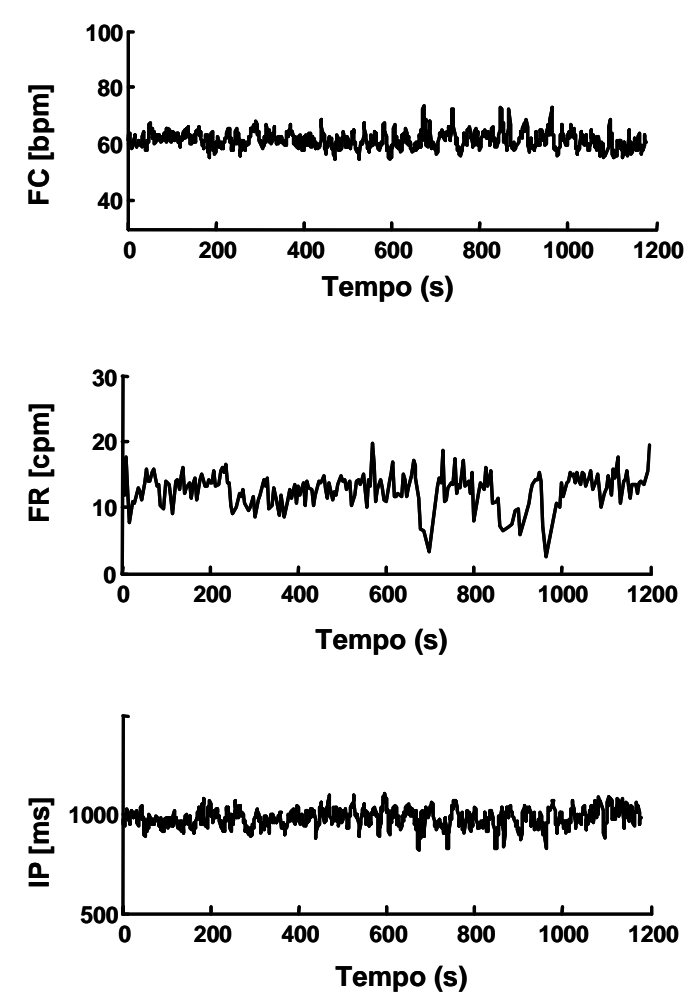

FIGURA 14 - $\underline{\text { Séries temporais de freqüência cardíaca (FC, bpm), freqüência }}$ respiratória (FR, cpm) e intervalo de pulso (IP, ms). 
Os gráficos individuais do intervalo de pulso e respectivos espectros de freqüência obtidos dos registros dos oito participantes do estudo constam do anexo IV.

5.2.1 Alterações nas flutuações rítmicas dos sinais do intervalo de pulso

A FIGURA 15 ilustra um exemplo de resultado da aplicação de análise espectral do IP obtido em um praticante de ioga antes (A) e APÓS A PRÁTICA de ioga (B).
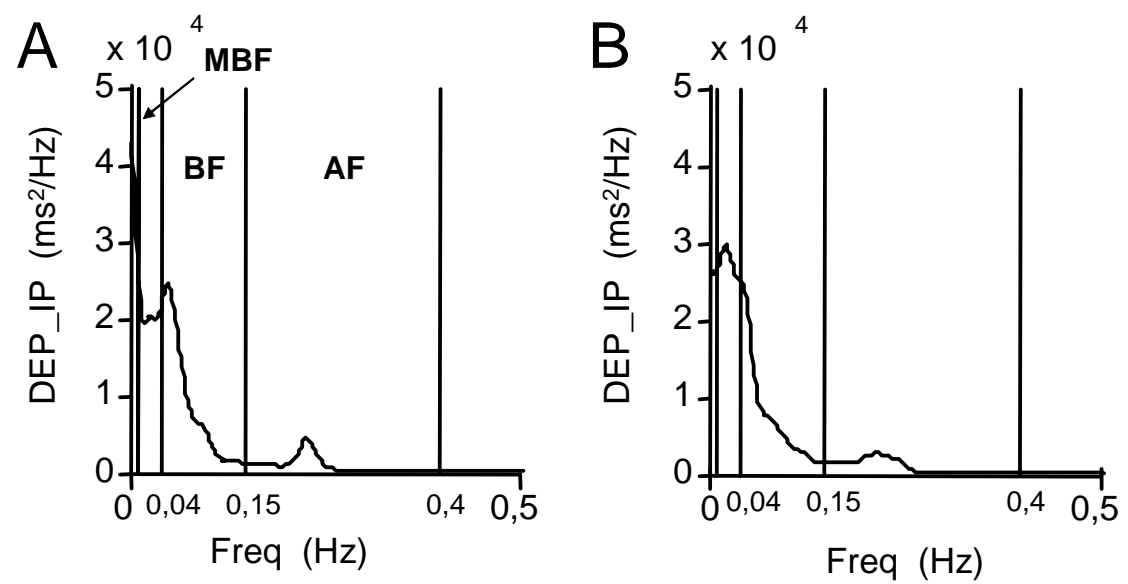

FIGURA 15- Espectro de potência $\left(\mathrm{ms}^{2} / \mathrm{Hz}\right)$ do intervalo de pulso nas faixas estudadas $(\mathrm{Hz})$ obtido de em um praticante de ioga na condição BASAL (A) e APÓS A PRÁTICA de ioga (B).

A seguir estão apresentados os resultados obtidos a partir da análise no domínio da freqüência.

A TABELA 10 apresenta os valores individuais e média (média \pm dp) das oscilações nas faixas de muito baixa freqüência (MBF), baixa freqüência (BF), alta freqüência (AF) do intervalo de pulso, potência total (PTOT) e variância total (VAR) e os valores médios e desvios padrão do grupo IOGA na condição BASAL. 
A TABELA 11 apresenta os valores individuais e média (média \pm dp) das oscilações nas faixas de muito baixa freqüência (MBF), baixa freqüência (BF), alta freqüência (AF) do intervalo de pulso, potência total (PTOT) e variância total (VAR) e os valores médios e desvios padrão do grupo CT na condição BASAL.

TABELA 10 - Valores individuais e média (média $\pm \mathrm{dp}$ ) da potência espectral do IP nas faixas MBF, BF, AF, PTOT e VAR do grupo IOGA na condição BASAL.

\begin{tabular}{cccccc}
\hline $\begin{array}{c}\text { IOGA } \\
\text { BASAL }\end{array}$ & $\begin{array}{c}\text { MBF } \\
\left(\mathrm{ms}^{2}\right)\end{array}$ & $\begin{array}{c}\mathrm{BF} \\
\left(\mathrm{ms}^{2}\right)\end{array}$ & $\begin{array}{c}\mathrm{AF} \\
\left(\mathrm{ms}^{2}\right)\end{array}$ & $\begin{array}{c}\text { PTOT } \\
\left(\mathrm{ms}^{2}\right)\end{array}$ & $\begin{array}{c}\text { VAR } \\
\left(\mathrm{ms}^{2}\right)\end{array}$ \\
\hline 1 & 1014,1 & 1036,8 & 1163,9 & 3196,8 & 3255,3 \\
2 & 1517,1 & 514,8 & 1073,2 & 3107,2 & 3090,4 \\
3 & 1993,8 & 2017,4 & 2382,1 & 6475,4 & 6601,4 \\
4 & 365,4 & 866,9 & 280,6 & 1499,6 & 1493,6 \\
5 & 1031,5 & 941,6 & 206,0 & 2148,9 & 2271,3 \\
6 & 3535,4 & 1024,4 & 582,5 & 5107,6 & 5471,9 \\
7 & 547,9 & 742,4 & 502,2 & 1795,5 & 1946,4 \\
8 & 1232,6 & 7936,4 & 3035,4 & 12419,6 & 11910,1 \\
média & 1404,7 & 1885,1 & 1153,2 & 4468,8 & 4505,1 \\
dp & 1002,5 & 2484,4 & 1032,9 & 3634,5 & 3470,9 \\
\hline
\end{tabular}

TABELA 11 - Valores individuais e média (média \pm dp) da potência espectral do IP nas faixas MBF, BF, AF, PTOT e VAR do grupo CT na condição BASAL.

\begin{tabular}{cccccc}
\hline $\begin{array}{c}\text { CT } \\
\text { BASAL }\end{array}$ & $\begin{array}{c}\text { MBF } \\
\left(\mathrm{ms}^{2}\right)\end{array}$ & $\begin{array}{c}\mathrm{BF} \\
\left(\mathrm{ms}^{2}\right)\end{array}$ & $\begin{array}{c}\text { AF } \\
\left(\mathrm{ms}^{2}\right)\end{array}$ & $\begin{array}{c}\text { PTOT } \\
\left(\mathrm{ms}^{2}\right)\end{array}$ & $\begin{array}{c}\text { VAR } \\
\left(\mathrm{ms}^{2}\right)\end{array}$ \\
\hline 1 & 684,8 & 1399,2 & 737,4 & 2816,6 & 2681,5 \\
2 & 312,6 & 198,6 & 231,9 & 742,5 & 747,9 \\
3 & 131,1 & 87,3 & 410,7 & 644,5 & 643,3 \\
4 & 1532,0 & 443,9 & 835,3 & 2805,1 & 2793,3 \\
5 & 605,9 & 637,3 & 1615,1 & 2887,6 & 2891,4 \\
6 & 728,8 & 907,5 & 206,2 & 1863,7 & 1977,0 \\
7 & 1215,2 & 131,1 & 37,2 & 1384,4 & 1535,7 \\
8 & 1168,1 & 5239,6 & 1570,8 & 8045,1 & 7910,8 \\
média & 797,3 & 1130,6 & 705,6 & 2648,7 & 2647,6 \\
dp & 475,8 & 1718,7 & 609,6 & 2363,3 & 2302,0 \\
\hline
\end{tabular}


A PTOT e a VAR apresentaram diferença significativa entre os grupos IOGA e CT e estão são ilustradas a seguir:

A FIGURA 16 ilustra a potência total (PTOT) nos grupos IOGA e CT nas condições BASAL e APÓS A PRÁTICA.

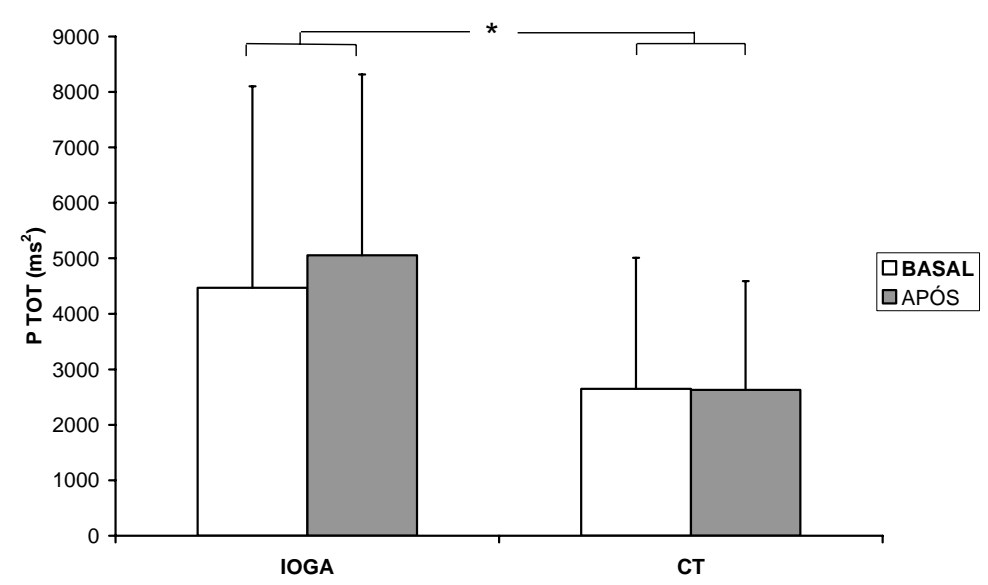

FIGURA 16 - $\underline{\text { Potência total (PTOT) nos grupos IOGA e CT nas condições }}$ BASAL e APÓS A PRÁTICA * $\mathrm{p}<0,05$.

A potência total do intervalo de pulso não apresenta diferença significativa para um mesmo grupo quando comparadas as condições BASAL e APÓS A PRÁTICA. No entanto, quando os grupos são comparados sem distinção entre as condições BASAL e APÓS A PRÁTICA, o grupo IOGA apresenta potência total maior $\left(4762,5 \pm 3349,4 \mathrm{~ms}^{2}\right)$ daquela encontrada para o grupo CT $\left(2638,2 \pm 2098,6 \mathrm{~ms}^{2}\right), \mathrm{p}=0,046$.

A variância da potência não apresenta diferença significativa para o mesmo grupo quando comparadas as condições BASAL e APÓS A PRÁTICA.. No entanto, há diferença entre os grupo quando as condições BASAL e APÓS A PRÁTICA são consideradas sem distinção. O valor da VAR no grupo IOGA $\left(4776,4 \pm 3262,0 \mathrm{~ms}^{2}\right)$ é maior em relação à VAR no grupo CT $(2648,4 \pm 2064,1$ $\left.\mathrm{ms}^{2}\right), \mathrm{p}=0,042$. 
A FIGURA 17 ilustra a variância (VAR) nos grupos IOGA e controle.

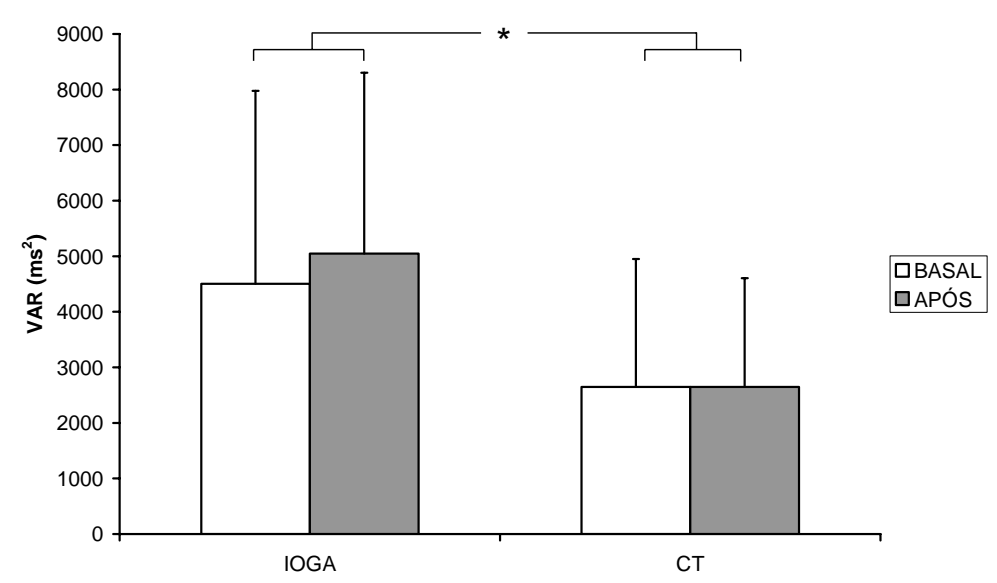

FIGURA 17 - Variância (VAR) nos grupos IOGA e CT nas condições BASAL e APÓS A PRÁTICA * $p<0,05$.

A TABELA 12 mostra os valores individuais e média (média $\pm \mathrm{dp}$ ) da potência espectral normalizada para as faixas de BF (BFn) e de AF (AFn) e razão entre baixa e alta freqüências normalizadas (BF/AF) do grupo IOGA na condição BASAL.

TABELA 12- Valores individuais e média (média \pm dp) da BFn, AFn e BF/AF no grupo IOGA na condição BASAL.

\begin{tabular}{cccc}
\hline $\begin{array}{c}\text { IOGA } \\
\text { BASAL }\end{array}$ & BFn & AFn & BF/AF \\
\hline 1 & 46,3 & 51,9 & 0,9 \\
2 & 32,7 & 68,2 & 0,5 \\
3 & 43,8 & 51,7 & 0,8 \\
4 & 76,8 & 24,9 & 3,1 \\
5 & 75,9 & 16,6 & 4,6 \\
6 & 52,9 & 30,1 & 1,8 \\
7 & 53,1 & 35,9 & 1,5 \\
8 & 74,3 & 28,4 & 2,6 \\
média & 57,0 & 38,5 & 2,0 \\
dp & 16,8 & 17,2 & 1,4 \\
\hline
\end{tabular}

A TABELA 13 mostra os valores individuais e média (média \pm dp) da potência espectral normalizada para as faixa de $\mathrm{BF}(\mathrm{BFn})$ e de $\mathrm{AF}$ ( $\mathrm{AFn}$ ) e razão entre baixa e alta freqüências normalizadas $(\mathrm{BF} / \mathrm{AF})$ do grupo do grupo controle (CT) na condição BASAL. 
TABELA 13 - Valores individuais e média (média \pm dp) da BFn, AFn e BF/AF no grupo CT na condição BASAL.

\begin{tabular}{cccc}
\hline CT & BFn & AFn & BF/AF \\
BASAL & & & 1,9 \\
\hline 1 & 70,1 & 36,9 & 0,9 \\
2 & 45,6 & 53,3 & 0,2 \\
3 & 17,1 & 80,2 & 0,5 \\
4 & 35,2 & 66,2 & 0,4 \\
5 & 27,9 & 70,7 & 4,4 \\
6 & 72,7 & 16,5 & 3,5 \\
7 & 40,9 & 11,6 & 3,3 \\
8 & 77,7 & 23,3 & 1,9 \\
média & 48,4 & 44,8 & 1,6 \\
dp & 22,6 & 26,4 & \\
\hline
\end{tabular}

Os valores absolutos obtidos a partir da análise espectral do intervalo de pulso para o período APÓS A PRÁTICA nos grupos IOGA e CT estão apresentados a seguir.

A TABELA 14 apresenta os valores individuais da potência espectral nas faixas de MBF, BF, AF, PTOT e VAR e os valores das médias e desvios padrão no grupo IOGA na condição APÓS A PRÁTICA.

TABELA 14 - Valores individuais e média (média \pm dp) MBF, BF e AF, PTOT e VAR no grupo IOGA na condição APÓS A PRÁTICA.

\begin{tabular}{cccccc}
\hline $\begin{array}{c}\text { IOGA } \\
\text { APÓS A }\end{array}$ & $\begin{array}{c}\text { MBF } \\
\left(\mathrm{ms}^{2}\right)\end{array}$ & $\begin{array}{c}\mathrm{BF} \\
\left(\mathrm{ms}^{2}\right)\end{array}$ & $\begin{array}{c}\text { AF } \\
\left(\mathrm{ms}^{2}\right)\end{array}$ & $\begin{array}{c}\text { PTOT } \\
\left(\mathrm{ms}^{2}\right)\end{array}$ & $\begin{array}{c}\text { VAR } \\
\left(\mathrm{ms}^{2}\right)\end{array}$ \\
\hline PRÁTICA & & & & & \\
\hline 1 & 1084,4 & 988,1 & 1223,2 & 3280,1 & 3320,1 \\
3 & 2213,6 & 3246,9 & 2365,7 & 7809,4 & 7722,0 \\
4 & 1654,6 & 2534,8 & 3098,8 & 7392,4 & 7845,7 \\
5 & 599,7 & 524,5 & 505,5 & 1620,3 & 1672,9 \\
6 & 1125,9 & 830,2 & 230,9 & 2151,0 & 2070,5 \\
7 & 4245,4 & 826,4 & 1585,7 & 6629,8 & 6417,0 \\
8 & 531,6 & 721,1 & 382,3 & 1632,0 & 1590,2 \\
média & 1325,6 & 6130,1 & 2437,9 & 9933,5 & 9743,4 \\
dp & 1597,6 & 1975,3 & 1478,7 & 5056,1 & 5047,7 \\
\hline
\end{tabular}


A TABELA 15 apresenta os valores individuais da potência espectral nas faixas de $\mathrm{MBF}, \mathrm{BF}, \mathrm{AF}, \mathrm{PTOT}$ e VAR e os valores das médias e desvios padrão no grupo CT na condição APÓS A PRÁTICA.

TABELA 15 - Valores individuais e média (média \pm dp) da potência espectral do IP nas faixas MBF, BF e AF, PTOT e VAR no grupo CT na condição APÓS A PRÁTICA.

\begin{tabular}{cccccc}
\hline $\begin{array}{c}\text { CT } \\
\text { APÓS A } \\
\text { PRÁTICA }\end{array}$ & $\begin{array}{c}\text { MBF } \\
\left(\mathrm{ms}^{2}\right)\end{array}$ & $\begin{array}{c}\mathrm{BF} \\
\left(\mathrm{ms}^{2}\right)\end{array}$ & $\begin{array}{c}\mathrm{AF} \\
\left(\mathrm{ms}^{2}\right)\end{array}$ & $\begin{array}{c}\text { PTOT } \\
\left(\mathrm{ms}^{2}\right)\end{array}$ & $\begin{array}{c}\text { VAR } \\
\left(\mathrm{ms}^{2}\right)\end{array}$ \\
\hline 1 & 798,3 & 993,7 & 1041,5 & 2831,7 & 2916,2 \\
2 & 320,9 & 167,6 & 131,7 & 615,4 & 633,5 \\
3 & 144,3 & 121,0 & 445,6 & 725,0 & 756,2 \\
4 & 3039,0 & 987,8 & 1210,8 & 5217,5 & 5171,8 \\
5 & 1665,9 & 783,3 & 1884,3 & 4392,8 & 4426,5 \\
6 & 799,5 & 1043,7 & 228,6 & 2110,8 & 2191,1 \\
7 & 270,9 & 82,20 & 68,5 & 424,2 & 388,1 \\
8 & 981,3 & 1852,3 & 1847,7 & 4703,9 & 4710,0 \\
média & 1002,5 & 753,9 & 857,3 & 2627,7 & 2649,2 \\
dp & 956,7 & 609,3 & 747,2 & 1962,8 & 1957,0 \\
\hline
\end{tabular}

A TABELA 16 mostra os valores individuais e média (média \pm dp) da potência espectral normalizada para as faixa de BFn e de AFn e razão BF/AF no grupo IOGA na condição APÓS A PRÁTICA

TABELA 16 - Valores individuais e média (média \pm dp) da BFn, AFn e BF/AF no grupo IOGA na condição APÓS A PRÁTICA.

\begin{tabular}{cccc}
\hline IOGA & BFn & AFn & BF/AF \\
APÓS A PRÁTICA & & & 0,8 \\
\hline 1 & 44,2 & 54,7 & 1,4 \\
2 & 58,9 & 42,9 & 0,8 \\
3 & 40,9 & 50,1 & 1,0 \\
4 & 48,9 & 47,1 & 3,6 \\
5 & 87,9 & 24,4 & 0,5 \\
6 & 38,1 & 73,0 & 1,9 \\
7 & 68,1 & 36,1 & 2,5 \\
8 & 72,8 & 29,0 & 1,6 \\
média & 57,5 & 44,7 & 1,0 \\
dp & 17,6 & 15,5 & \\
\hline
\end{tabular}


A TABELA 17 mostra os valores individuais e média (média \pm dp) da potência espectral normalizada para as faixa de BFn e de AFn e razão BF/AF no grupo CT na condição APÓS A PRÁTICA

\begin{tabular}{cccc}
\hline CT & BFn & AFn & BF/AF \\
APÓS A PRÁTICA & & & \\
\hline 1 & 46,9 & 49,2 & 1,0 \\
2 & 53,6 & 42,1 & 1,3 \\
3 & 19,8 & 72,8 & 0,3 \\
4 & 46,3 & 56,8 & 0,8 \\
5 & 28,4 & 68,3 & 0,4 \\
6 & 75,0 & 16,4 & 4,6 \\
7 & 70,2 & 58,4 & 1,2 \\
8 & 49,7 & 49,6 & 1,0 \\
média & 48,7 & 51,7 & 1,3 \\
dp & 18,7 & 17,5 & 1,4 \\
\hline
\end{tabular}

TABELA 17 - Valores individuais e média (média \pm dp) da BFn, AFn, BF/AF no grupo CT na condição APÓS A PRÁTICA

Não foram encontradas diferenças significativas entre os grupos IOGA e CT para os valores da potência espectral nas faixas de MBF $(1501,2 \pm$ $1073,0 \mathrm{~ms}^{2}$ e $\left.899,9 \pm 737,6 \mathrm{~ms}^{2}, \mathrm{p}=0,083\right), \mathrm{BF}\left(1930,2 \pm 2155,9 \mathrm{~ms}^{2}\right.$ e $942,2 \pm$ $\left.1260,8 \mathrm{~ms}^{2} \mathrm{p}=0,136\right)$, e $\operatorname{AF}\left(1316,0 \pm 1033,0 \mathrm{~ms}^{2}\right.$ e $781,5 \pm 663,4 \mathrm{~ms}^{2} ; \mathrm{p}=$ $0,100)$, assim como para as potência normalizadas BFn $(57,2 \pm 16,6$; $\mathrm{p}=\mathrm{e} 48,6$ $\pm 20,0 ; p=0,209), \operatorname{AFn}(41,6 \pm 16,1$ e 48,3 $\pm 21,9 ; p=0,342)$ ou para o índice $\mathrm{BF} / \mathrm{AF}(1,8 \pm 1,2$ e $1,6 \pm 1,5 ; \mathrm{p}=0,742)$.

Do grupo inicial de oito indivíduos praticantes de ioga, foi selecionado um sub-grupo (IOGAativ), consistindo de indivíduos que praticavam atividades físicas além da ioga e que possuíam freqüência respiratória maior que dez ciclos por minuto, correspondendo aos indivíduos 1 , 2, 3, 6 (TABELA 3). Neste grupo a média de idade é de 35,8 $\pm 2,2$ anos, estatura média $1,70 \pm 0,1 \mathrm{~m}$, massa corporal média de 59,5 \pm 7,9 $\mathrm{kg}$ e índice de massa corpórea (IMC) médio de $21,8 \pm 1,0 \mathrm{~kg} / \mathrm{m}^{2}$. 
De maneira semelhante, do grupo de voluntários não praticantes de ioga foi selecionado um sub-grupo (CTativ) de indivíduos que praticavam atividades físicas e que possuíam freqüência respiratória maior que dez cpm, correspondendo aos indivíduos 2, 3, 4, 6 (TABELA 4). Neste grupo a média de idade é de 40,8 $\pm 3,2$ anos, estatura média 1,60 $\pm 0,10 \mathrm{~m}$, massa corporal média de $67,50 \pm 15,2 \mathrm{~kg}$ e IMC médio de $25,1 \pm 2,3 \mathrm{~kg} / \mathrm{m}^{2}$.

5.3 Análise no domínio do tempo para os grupos IOGAativ e CTativ.

Serão apresentados a seguir os resultados obtidos no domínio do tempo para os grupos IOGAativ e CTativ.

A FIGURA 18 ilustra a freqüência cardíaca (FC) nos grupos IOGAativ e CTativ.

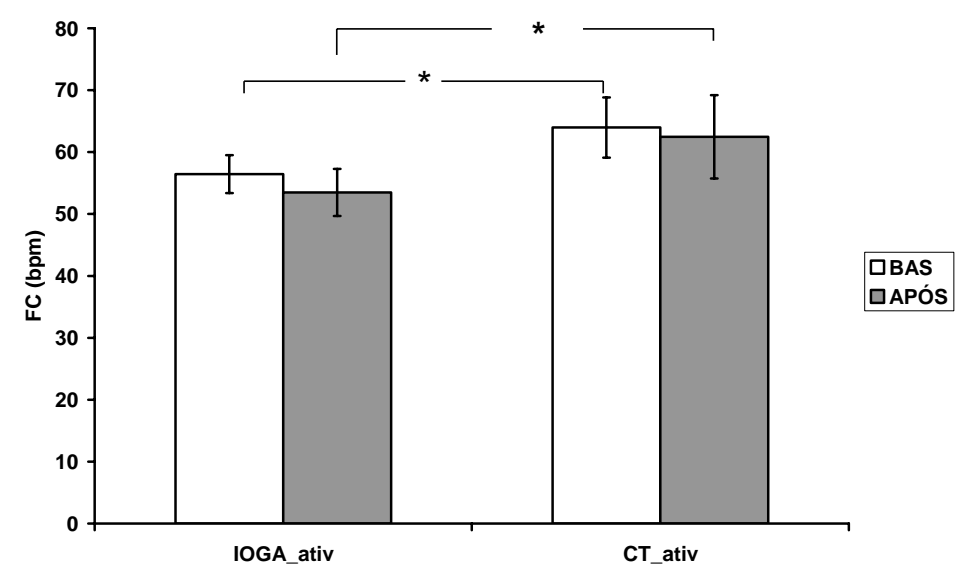

FIGURA 18 - Freqüência cardíaca (FC) nos grupos IOGAativ e CTativ nas condições BASAL e APÓS A PRÁTICA * $\mathrm{p}<0,05$.

Para o mesmo grupo, não houve diferença na freqüência cardíaca entre as condições BASAL e APÓS A PRÁTICA. Entre os grupos, houve diferença entre as condições BASAL e APÓS A PRÁTICA, sendo o valor da FC na condição BASAL no grupo IOGAativ $(56,5 \pm 3,1 \mathrm{bpm})$ menor em relação à FC no grupo CTativ $(64,0 \pm 4,9$ bpm; $p=0,048)$. Da mesma forma, na condição 
APÓS A PRÁTICA, o valor da FC no grupo IOGAativ $(53,5 \pm 3,8 \mathrm{bpm})$ foi menor do que a FC $(62,5 \pm 6,7 \mathrm{bpm} ; \mathrm{p}=0,021)$ no grupo CTativ.

A FIGURA 19 ilustra o desvio padrão da freqüência cardíaca (DFC) nos grupos IOGAativ e CTativ.

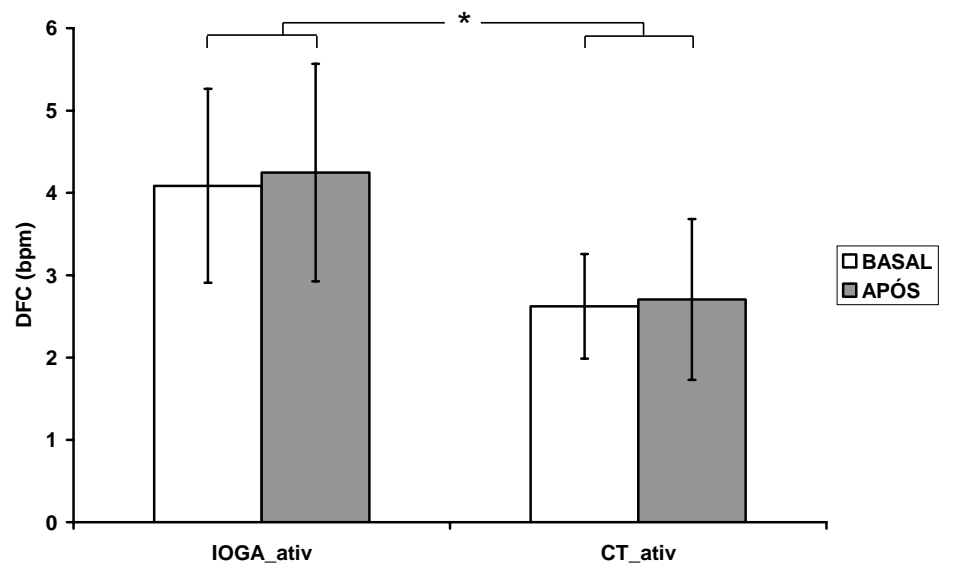

FIGURA 19 - Desvio padrão da freqüência cardíaca (DFC) nos grupos IOGAativ e CTativ nas condições BASAL e APÓS A PRÁTICA * $p<0,05$.

Para o mesmo grupo não houve diferença no DFC entre os períodos BASAL e APÓS A PRÁTICA. Independentemente da fase, para o grupo de praticantes de ioga, o DFC foi maior $(4,2 \pm 1,2 \mathrm{bpm})$ do que o DFC no grupo controle $(2,7 \pm 0,8 \mathrm{bpm}, \mathrm{p}=0,015)$.

Não houve diferença significativa para o intervalo de pulso entre as fases BASAL e APÓS A PRÁTICA em um mesmo grupo. No entanto, houve diferença entre grupos no período APÓS A PRÁTICA, sendo o valor do IP no grupo IOGAativ $(1132,9 \pm 80,6 \mathrm{~ms})$ maior em relação ao valor do IP no grupo CTativ $(971,7 \pm 115,9 \mathrm{~ms}, \mathrm{p}=0,020)$. 
A FIGURA 20 ilustra o intervalo de pulso (IP) nos grupos IOGAativ e CTativ.

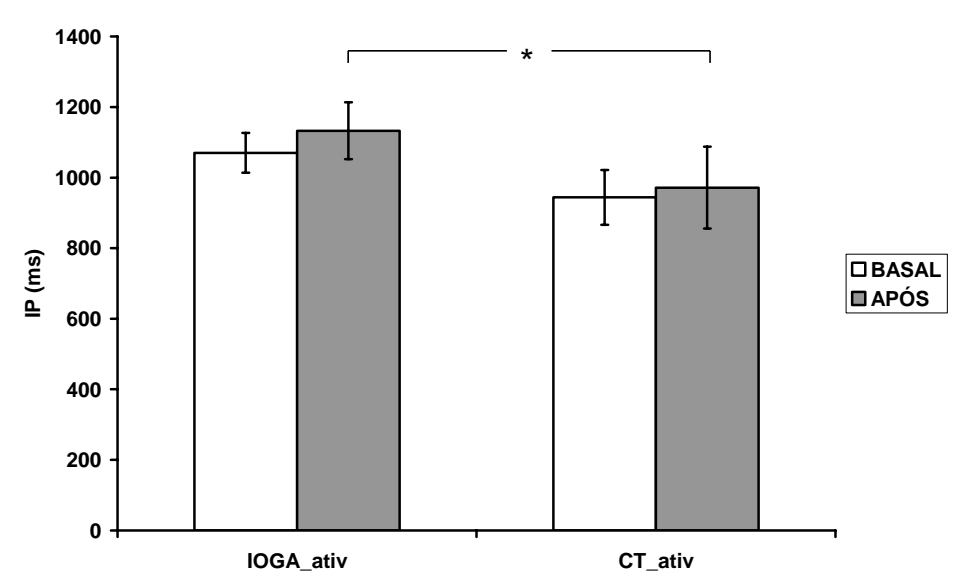

FIGURA 20 - Intervalo de pulso (IP) nos grupos IOGAativ e CTativ nas condições BASAL e APÓS A PRÁTICA * $p<0,05$.

A FIGURA 21 ilustra o desvio padrão do intervalo de pulso (DIP) nos grupos IOGAativ e CTativ.

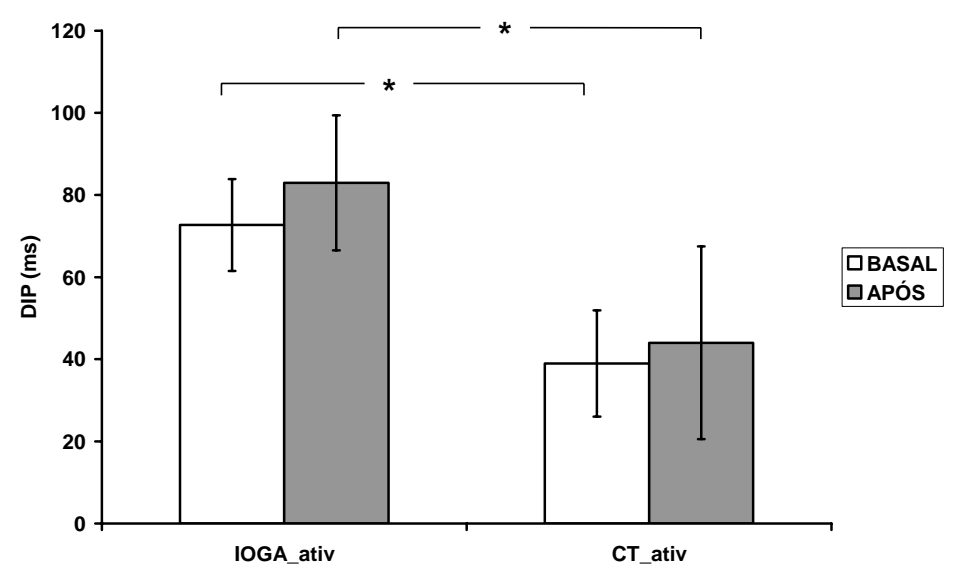

FIGURA 21- Desvio do intervalo de pulso (DIP) nos grupos IOGAativ e CTativ nas condições BASAL e APÓS A PRÁTICA * p <0,05.

Num mesmo grupo, não houve diferença no desvio padrão do intervalo de pulso entre as condições BASAL e APÓS A PRÁTICA. No entanto, entre os grupos e no período BASAL, o DIP foi maior no grupo IOGAativ $(72,7 \pm 11,2 \mathrm{~ms})$ comparado grupo CTativ $(38,9 \pm 13,0 \mathrm{~ms} ; \mathrm{p}=$ 
0,014). Da mesma forma, na condição APÓS A PRÁTICA, o valor do DIP foi maior no grupo IOGAativ $(83,0 \pm 16,4 \mathrm{~ms})$ em relação ao grupo CTativ $(44,0 \pm$ $23,5 \mathrm{~ms} ; \mathrm{p}=0,006)$. Não foi observada diferença significativa para freqüência respiratória $(14,0 \pm 1,5 \mathrm{cpm}$ e $14,3 \pm 1,3 \mathrm{cpm} ; \mathrm{p}=0,469)$ e desvio padrão da freqüência respiratória $(2,5 \pm 1,2$ e 2,4 $\pm 0,9 \mathrm{cpm} ; \mathrm{p}=0,556)$ entre os grupos IOGAativ e CTativ.

Os valores do $\mathrm{DFC}$ dos grupos $\mathrm{IOGA}_{\text {ativ }}$ e $\mathrm{CT}_{\text {ativ }}$ não foram diferentes nas condições BASAL $(4,0 \pm 1,2$ bpm e 2,6 $\pm 0,6$ bpm; $p=0,073)$ e APÓS A PRÁTICA $(4,2 \pm 1,3$ e 2,7 $\pm 1,0$ bpm; $p=0,065)$, assim como o IP na condição BASAL $(1070,3 \pm 56,6 \mathrm{~ms}$ e $943,8 \pm 77,7 \mathrm{~ms} ; \mathrm{p}=0,058)$.

5.4 Análise no domínio da freqüência para os grupos IOGAativ e CTativ

A seguir estão apresentados os resultados no domínio da freqüência para os grupos IOGAativ e CTativ.

A TABELA 18 e a TABELA 19 mostram os valores individuais da potência espectral nas faixas de MBF, BF, AF, PTOT e VAR e os valores das médias e desvios padrão no grupo IOGAativ e CTativ na condição BASAL.

TABELA 18 - Valores individuais e média (média \pm dp) da MBF, BF e AF, PTOT e VAR no grupo IOGAativ na condição BASAL.

\begin{tabular}{cccccc}
\hline $\begin{array}{c}\text { IOGAativ } \\
\text { BASAL }\end{array}$ & $\begin{array}{c}\text { MBF } \\
\left(\mathrm{ms}^{2}\right)\end{array}$ & $\begin{array}{c}\mathrm{BF} \\
\left(\mathrm{ms}^{2}\right)\end{array}$ & $\begin{array}{c}\text { AF } \\
\left(\mathrm{ms}^{2}\right)\end{array}$ & $\begin{array}{c}\text { PTOT } \\
\left(\mathrm{ms}^{2}\right)\end{array}$ & $\begin{array}{c}\text { VAR } \\
\left(\mathrm{ms}^{2}\right)\end{array}$ \\
\hline 1 & 1014,1 & 1036,8 & 1163,9 & 3196,8 & 3255,3 \\
2 & 1517,1 & 514,8 & 1073,2 & 3107,2 & 3090,4 \\
3 & 1993,8 & 2017,4 & 2382,1 & 6475,4 & 6601,4 \\
6 & 3535,4 & 1024,4 & 582,5 & 5107,6 & 5471,9 \\
média & 2015,1 & 1148,3 & 1300,4 & 4471,7 & 4604,8 \\
dp & 1089,6 & 628,3 & 765,0 & 1623,4 & 1717,8 \\
\hline
\end{tabular}


TABELA 19 - Valores individuais e média (média \pm dp) da MBF, BF e AF, PTOT e VAR no grupo CTativ na condição BASAL.

\begin{tabular}{cccccc}
\hline $\begin{array}{c}\text { CTativ } \\
\text { BASAL }\end{array}$ & $\begin{array}{c}\text { MBF } \\
\left(\mathrm{ms}^{2}\right)\end{array}$ & $\begin{array}{c}\text { BF } \\
\left(\mathrm{ms}^{2}\right)\end{array}$ & $\begin{array}{c}\text { AF } \\
\left(\mathrm{ms}^{2}\right)\end{array}$ & $\begin{array}{c}\text { PTOT } \\
\left(\mathrm{ms}^{2}\right)\end{array}$ & $\begin{array}{c}\text { VAR } \\
\left(\mathrm{ms}^{2}\right)\end{array}$ \\
\hline 2 & 312,6 & 198,6 & 231,9 & 742,5 & 747,9 \\
3 & 131,1 & 87,3 & 410,7 & 644,5 & 643,3 \\
4 & 1532,0 & 443,9 & 835,3 & 2805,1 & 2793,3 \\
6 & 728,8 & 907,5 & 206,2 & 1863,7 & 1977,0 \\
média & 676,1 & 409,3 & 421,0 & 1514,0 & 1540,4 \\
dp & 623,0 & 364,0 & 290,8 & 1023,1 & 1031,7 \\
\hline
\end{tabular}

A TABELA 20 e a TABELA 21 mostram os valores individuais e média (média $\pm \mathrm{dp}$ ) das potências espectrais normalizadas nas faixas BFn, AFn e a razão BF/AF do grupo IOGAativ e CTativ na condição BASAL.

TABELA 20 - Valores individuais e média (média \pm dp) da BFn, AFn e BF/AF do grupo IOGAativ na condição BASAL.

\begin{tabular}{cccc}
\hline $\begin{array}{c}\text { IOGAativ } \\
\text { BASAL }\end{array}$ & BFn & AFn & BF/AF \\
\hline 1 & 46,3 & 51,9 & 0,9 \\
2 & 32,7 & 68,2 & 0,5 \\
3 & 43,8 & 51,7 & 0,8 \\
6 & 52,9 & 30,1 & 1,8 \\
média & 43,9 & 50,5 & 1,0 \\
dp & 8,4 & 15,6 & 0,5 \\
\hline
\end{tabular}

TABELA 21 - Valores individuais e média (média \pm dp) da BFn, AFn e BF/AF no grupo CTativ na condição BASAL.

\begin{tabular}{cccc}
\hline $\begin{array}{c}\text { CTativ } \\
\text { BASAL }\end{array}$ & BFn & AFn & BF/AF \\
\hline 2 & 45,6 & 53,3 & 0,9 \\
3 & 17,1 & 80,2 & 0,2 \\
4 & 35,2 & 66,2 & 0,5 \\
6 & 72,7 & 16,5 & 4,4 \\
média & 42,6 & 54,1 & 1,5 \\
dp & 23,3 & 27,3 & 2,0 \\
\hline
\end{tabular}


A TABELA 22 e a TABELA 23 mostram os valores individuais das oscilações nas faixas de $\mathrm{MBF}, \mathrm{BF}$ e AF, PTOT, VAR e os valores médios e desvios padrão do grupo IOGAativ e CTativ na condição APÓS A PRÁTICA. TABELA 22 - Valores individuais e média (média $\pm \mathrm{dp}$ ) da MBF, BF, AF, PTOT e VAR no grupo IOGAativ na condição APÓS A PRÁTICA.

\begin{tabular}{cccccc}
\hline $\begin{array}{c}\text { IOGAativ } \\
\text { APÓS A }\end{array}$ & $\begin{array}{c}\mathrm{MBF} \\
\left(\mathrm{ms}^{2}\right)\end{array}$ & $\begin{array}{c}\mathrm{BF} \\
\left(\mathrm{ms}^{2}\right)\end{array}$ & $\begin{array}{c}\mathrm{AF} \\
\left(\mathrm{ms}^{2}\right)\end{array}$ & $\begin{array}{c}\text { PTOT } \\
\left(\mathrm{ms}^{2}\right)\end{array}$ & $\begin{array}{c}\text { VAR } \\
\left(\mathrm{ms}^{2}\right)\end{array}$ \\
\hline PRÁTICA & & & & & 3320,1 \\
2 & 1084,4 & 988,1 & 1223,2 & 3280,1 & 7722,0 \\
3 & 2213,6 & 3246,9 & 2365,7 & 7809,4 & 7845,7 \\
6 & 1654,6 & 2534,8 & 3098,8 & 7392,4 & 6417,0 \\
média & 4245,4 & 826,4 & 1585,7 & 6629,8 & 6326,2 \\
dp & 2299,5 & 1899,1 & 2068,3 & 6277,9 & 2105,7 \\
\hline
\end{tabular}

TABELA 23 - Valores individuais e média (média $\pm \mathrm{dp}$ ) da MBF, BF, AF, PTOT e VAR no grupo CTativ na condição APÓS A PRÁTICA.

\begin{tabular}{cccccc}
\hline $\begin{array}{c}\text { CTativ } \\
\text { APÓS A } \\
\text { PRÁTICA }\end{array}$ & $\begin{array}{c}\text { MBF } \\
\left(\mathrm{ms}^{2}\right)\end{array}$ & $\begin{array}{c}\mathrm{BF} \\
\left(\mathrm{ms}^{2}\right)\end{array}$ & $\begin{array}{c}\text { AF } \\
\left(\mathrm{ms}^{2}\right)\end{array}$ & $\begin{array}{c}\text { PTOT } \\
\left(\mathrm{ms}^{2}\right)\end{array}$ & $\begin{array}{c}\text { VAR } \\
\left(\mathrm{ms}^{2}\right)\end{array}$ \\
\hline 2 & 320,9 & 167,6 & 131,7 & 615,4 & 633,5 \\
3 & 144,3 & 121,0 & 445,6 & 725,0 & 756,2 \\
4 & 3039,0 & 987,8 & 1210,8 & 5217,5 & 5171,8 \\
6 & 799,5 & 1043,7 & 228,6 & 2110,8 & 2191,1 \\
média & 1075,9 & 580,0 & 504,2 & 2167,2 & 2188,2 \\
dp & 1337,7 & 504,0 & 489,0 & 2144,4 & 2111,0 \\
\hline
\end{tabular}

A TABELA 24 e a TABELA 25 mostram os valores individuais da $\mathrm{BFn}, \mathrm{AFn}$ e razão $\mathrm{BF} / \mathrm{AF}$ e os valores médios e desvios padrão do grupo IOGAativ e CTativ na condição APÓS A PRÁTICA. 
TABELA 24 - Valores individuais e média (média \pm dp) da BFn, AFn e BF/AF no grupo IOGAativ na condição APÓS A PRÁTICA.

\begin{tabular}{cccc}
\hline $\begin{array}{c}\text { IOGAativ } \\
\text { APÓS A PRÁTICA }\end{array}$ & BFn & AFn & BF/AF \\
\hline 1 & 44,2 & 54,7 & 0,8 \\
2 & 58,9 & 42,9 & 1,4 \\
3 & 40,9 & 50,1 & 0,8 \\
6 & 38,1 & 73,0 & 0,5 \\
média & 45,5 & 55,2 & 0,9 \\
dp & 9,3 & 12,8 & 0,4 \\
\hline
\end{tabular}

TABELA 25 - Valores individuais e média (média \pm dp) da BFn, AFn e BF/AF no grupo CTativ na condição APÓS A PRÁTICA.

\begin{tabular}{cccc}
\hline $\begin{array}{c}\text { CTativ } \\
\text { APÓS A PRÁTICA }\end{array}$ & BFn & AFn & BF/AF \\
\hline 2 & 53,6 & 42,1 & 1,3 \\
3 & 19,8 & 72,8 & 0,3 \\
4 & 46,3 & 56,8 & 0,8 \\
6 & 75,0 & 16,4 & 4,6 \\
média & 48,7 & 47,0 & 1,7 \\
dp & 22,8 & 23,9 & 1,9 \\
\hline
\end{tabular}

A seguir estão ilustrados os resultados obtidos da análise espectral do intervalo de pulso nos grupos IOGAativ e CTativ.

Para um mesmo grupo não houve diferença na faixa de muito baixa freqüência da potência espectral entre as condições BASAL e APÓS A PRÁTICA. No entanto, a potência espectral do intervalo de pulso na MBF foi maior no grupo IOGAativ $\left(2157,3 \pm 1159,4 \mathrm{~ms}^{2}\right)$ em relação ao grupo CTativ $\left(876,0 \pm 989,4 \mathrm{~ms}^{2}\right), \mathrm{p}=0,045$. 
A FIGURA 22 ilustra a potência espectral na faixa de muito baixa freqüência (MBF) do IP nos grupos IOGAativ e CTativ.

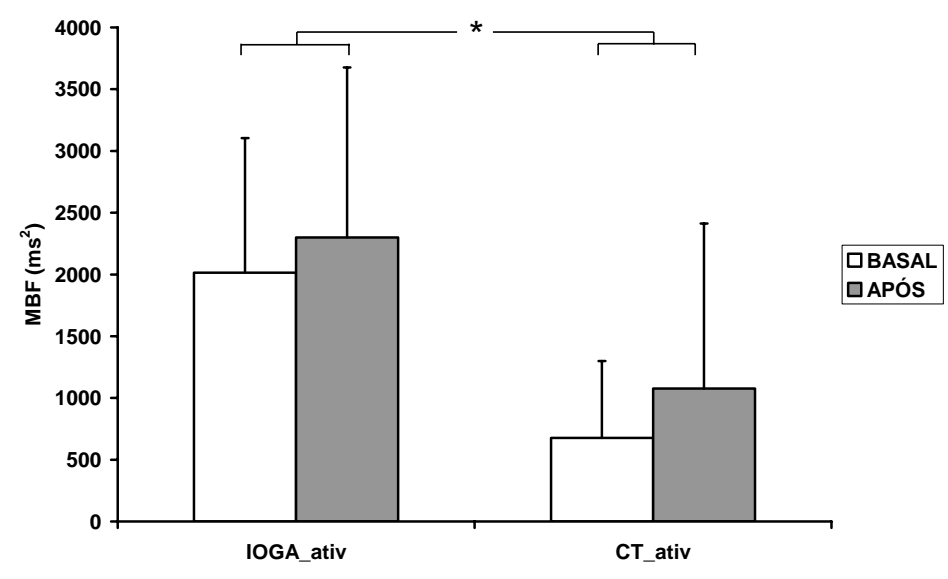

FIGURA 22 - Potência espectral na faixa de muito baixa freqüência (MBF) no grupo IOGAativ e CTativ nas condições BASAL e APÓS A PRÁTICA * $\mathrm{p}<0,05$.

A FIGURA 23 ilustra a potência espectral na faixa de baixa freqüência (BF) do IP nos grupos IOGAativ e CTativ.

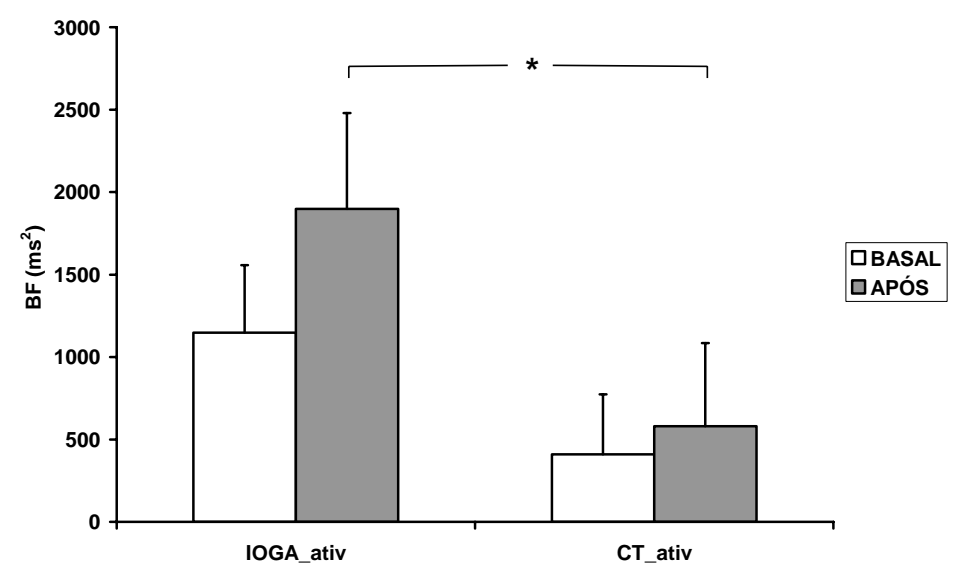

FIGURA 23 - Potência espectral na faixa de baixa freqüência (BF) nos grupos IOGAativ CTativ, nas condições BASAL e APÓS A PRÁTICA $* \mathrm{p}<0,05$.

Para o mesmo grupo não houve diferença na potência espectral na faixa de baixa freqüência do intervalo de pulso, quando comparadas as 
condições BASAL e APÓS A PRÁTICA. No entanto, quando comparados os grupos e para o período APÓS A PRÁTICA, o valor da potência em BF foi maior no grupo IOGAativ $\left(1899,0 \pm 580,0 \mathrm{~ms}^{2}\right)$ comparado ao grupo CTativ. $\left(580,0 \pm 504,0 \mathrm{~ms}^{2}\right), \mathrm{p}=0,027$.

A FIGURA 24 ilustra a potência espectral na faixa de alta freqüência (AF) do IP nos grupos IOGAativ e CTativ.

Não houve diferença para a potência espectral na faixa de alta freqüência para o mesmo grupo. No entanto, entre grupos e para o período APÓS A PRÁTICA, o valor da AF foi maior no grupo IOGAativ (2068,3 \pm $\left.836,1 \mathrm{~ms}^{2}\right)$ comparado ao grupo CTativ $\left(504,2 \pm 489,0 \mathrm{~ms}^{2}\right), \mathrm{p}=0,004$.

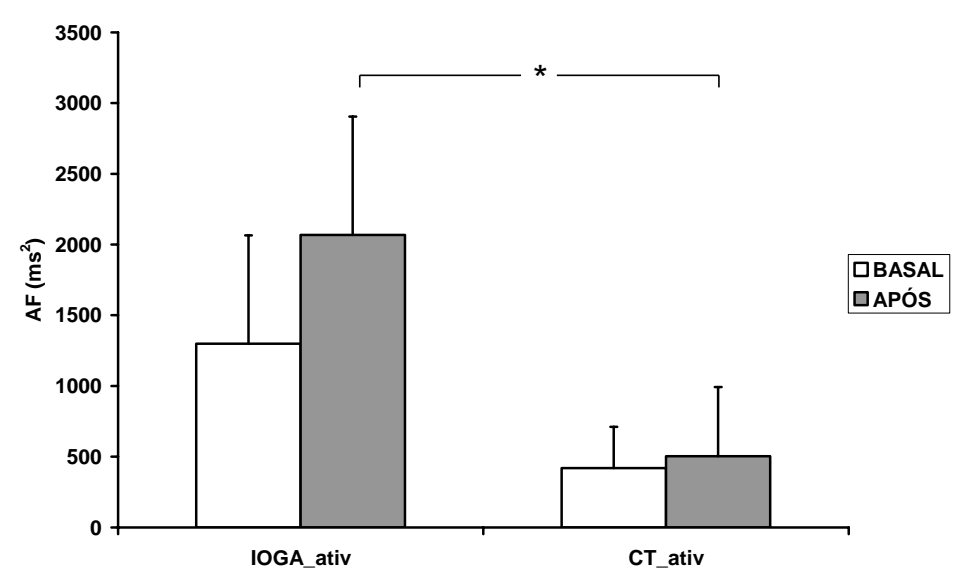

FIGURA 24 - Potência espectral na faixa de alta freqüência (AF) nos grupos IOGAativ CTativ, nas condições BASAL e APÓS A PRÁTICA * $p<0,05$.

A FIGURA 25 ilustra a potência espectral total (PTOT) do IP nos grupos IOGAativ e CTativ. Não houve diferença entre as condições BASAL ou APÓS A PRÁTICA para o mesmo grupo. No entanto, houve diferença entre os grupos em ambas as fases. Na condição BASAL a PTOT no grupo IOGAativ é maior $\left(4471,7 \pm 1623,4 \mathrm{~ms}^{2}\right)$ em relação ao CTativ na mesma condição (1514,0 $\left.\pm 1023 \mathrm{~ms}^{2} ; \mathrm{p}=0,036\right)$. Na condição APÓS A PRÁTICA, a PTOT no grupo 
IOGAativ foi também maior $\left(6277,9 \pm 2057,4 \mathrm{~ms}^{2}\right)$ em relação à PTOT na mesma condição no grupo CTativ $\left(2167,2 \pm 2144,4 \mathrm{~ms}^{2} ; \mathrm{p}=0,006\right)$.

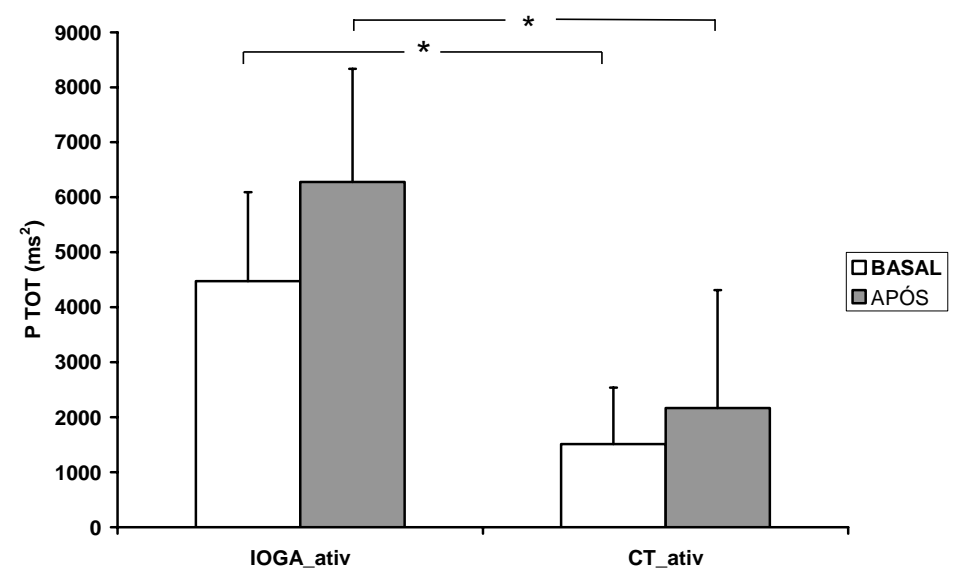

FIGURA 25 - Potência total (PTOT) do intervalo de pulso nos grupos IOGAativ CTativ nas condições BASAL e APÓS A PRÁTICA * $p<0,05$. A FIGURA 26 ilustra a variância (VAR) da potência total nos grupos IOGAativ e CTativ.

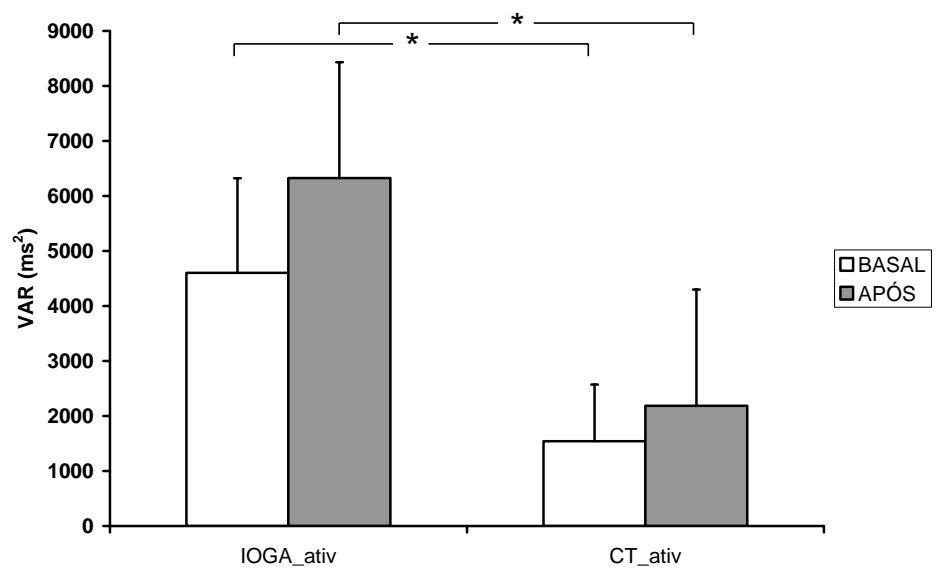

FIGURA 26 - Variância (VAR) da potência total nos grupos IOGAativ e CTativ nas condições BASAL e APÓS A PRÁTICA * $p<0,05$.

Não houve diferença para a variância da potência total entre as condições BASAL ou APÓS A PRÁTICA para o mesmo grupo. No entanto, há diferença entre os grupos em ambas as fases, sendo a VAR da potência total na condição BASAL, para o grupo IOGAativ $\left(4604,8 \pm 1717,8 \mathrm{~ms}^{2}\right)$ maior, 
comparada ao grupo CTativ $\left(1540,4 \pm 1031,7 \mathrm{~ms}^{2} ; \mathrm{p}=0,033\right)$. Considerando-se a condição APÓS A PRÁTICA, o valores obtido da VAR no grupo IOGAativ $\left(6326,2 \pm 2105,7 \mathrm{~ms}^{2}\right)$ foi também maior ao valor no grupo CTativ $(2188,2 \pm$ $\left.2111,0 \mathrm{~ms}^{2} ; \mathrm{p}=0,007\right)$. Não houve diferença entre os grupos IOGAativ e CTativ para a potência espectral na faixa de BFn $(44,7 \pm 8,2$ e 45,7 $\pm 21,6 ; \mathrm{p}=$ $0,916), \operatorname{AFn}(52,8 \pm 13,5$ e $50,5 \pm 24,1 ; p=0,830)$ e na razão $\operatorname{BF} / \operatorname{AF}(0,9 \pm 0,4$ e $1,6 \pm 1,8 ; p=0,352)$.

A TABELA 26 apresenta o resumo dos resultados obtidos na comparação entre os grupos IOGA x CT e entre os grupos IOGAativ X CTativ nos domínios do tempo e da freqüência. As alterções indicadas na tabela compreendem as diferenças encontradas no grupo IOGA ou IOGAativ em relação aos respectivos controles.

TABELA 26 - Resumo dos resultados

\begin{tabular}{|c|c|}
\hline IOGA x CT & IOGAativ x CTativ \\
\hline$\downarrow$ Freqüência cardíaca & $\begin{array}{ccccc}\downarrow & \text { Freqüência cardíaca } & \text { (BASAL e APÓS A } \\
\text { PRÁTICA) } & & & & \\
\end{array}$ \\
\hline$\uparrow$ Intervalo de pulso & $\uparrow$ Desvio padrão da freqüência cardíaca \\
\hline$\uparrow$ Desvio padrão do intervalo de pulso & $\uparrow$ Intervalo de pulso (APÓS A PRÁTICA) \\
\hline$\uparrow$ Potência total & $\begin{array}{l}\uparrow \text { Desvio padrão do intervalo de pulso (BASAL e } \\
\text { APÓS A PRÁTICA) }\end{array}$ \\
\hline$\uparrow$ Variância do intervalo de pulso & $\begin{array}{l}\uparrow \text { Potência espectral do intervalo de pulso na faixa de } \\
\text { muito baixa freqüência } \\
\uparrow \text { Potência espectral do intervalo de pulso na faixa de } \\
\text { baixa freqüência (APÓS A PRÁTICA) } \\
\uparrow \text { Potência espectral do intervalo de pulso na faixa de } \\
\text { alta freqüência (APÓS A PRÁTICA) } \\
\uparrow \text { Potência total (BASAL e APÓS A PRÁTICA) } \\
\uparrow \text { Variância do intervalo de pulso (BASAL e APÓS A } \\
\text { PRÁTICA) }\end{array}$ \\
\hline
\end{tabular}


A ioga tem-se popularizado como uma prática de combate ao estresse. Os estudos científicos acerca dos efeitos fisiológicos da prática da ioga tiveram início na década de 1920, na Índia, através do Instituto Kaivalyadhama, com uma publicação trimestral de estudos conduzidos no próprio Instituto. Outros estudos nos Estados Unidos e Europa surgiram na década de 1960. No Brasil a abordagem científica da prática da ioga foi introduzida pela prof ${ }^{\mathrm{a}}$. Ignez Novaes Romeu a partir de 1968 (Diário Popular, 12/05/1968). Estudos recentes desenvolvidos no Brasil utilizaram a prática da ioga (asanas e pranayamas) como procedimento complementar na assistência a pacientes neuróticos (SOUZA, 1999) e a prática de relaxamento (savasana) em pacientes hipertensos (SANTAELLA, 2003). Outro estudo observou movimento respiratório otimizado em mulheres idosas praticantes de ioga (LEITE, 1999) demonstrando seus benefícios. É possível que o misticismo normalmente atribuído à ioga dificulte a inclusão desta prática como terapia complementar ou aplicada em diferentes campos. O objetivo deste estudo foi avaliar possíveis efeitos agudos e crônicos da prática da ioga (asanas) a partir da análise do eletrocardiograma e respirograma, registrados de maneira não invasiva. Foram estudados praticantes de ioga (IOGA, $n=8)$ e não praticantes controles $(\mathrm{CT}, \mathrm{n}=8)$, com idade entre $30 \mathrm{e}$ 45 anos. O grupo CTativ apresentou faixa etária maior $(\mathrm{p}=0,04)$ quando comparado ao grupo IOGAativ. A faixa etária influencia a variabilidade da FC de maneira inversa (ANTELMI, 2004) e pode ter contribuído para a menor VFC encontrada no grupo CTativ. O grupo IOGA realizou a prática de uma seqüência de posturas enquanto o grupo CT realizou uma seqüência de movimentos que simulavam as alterações posturais encontradas na prática dos asanas. As seqüências de asanas ou movimentos foram precedidas e sucedidas por relaxamento conduzido. $\mathrm{O}$ eletrocardiograma e o respirograma foram registrados 
durante 20 minutos antes e após os asanas ou simulação. O respirograma foi analisado no domínio do tempo e o eletrocardiograma, no domínio do tempo e da freqüência. A análise em freqüência utilizou a técnica da transformada rápida de Fourier que permite estudar a variabilidade da freqüência cardíaca e flutuações do sinal do intervalo de pulso relacionadas ao controle autonômico da freqüência cardíaca (USHIZIMA, 2000). O uso da TRF para análise da variabilidade da freqüência cardíaca está amplamente validado (MALLIANI, PAGANI, LOMBARDI \& CERUTTI, 1991) e fornece resultados comparáveis aos da análise com algoritmos baseados em modelos auto-regressivos (Di RIENZO, MANCIA, PARATI, PEDOTTI \& ZANCHETTI, 1993; PARATI, SAUL, Di RIENZO \& MANCIA, 1995).

Os resultados encontrados indicam que a FC é menor no grupo IOGA $(58,9 \pm 6,6 \mathrm{bpm})$ comparado ao CT $(64,3 \pm 5,6 \mathrm{bpm})$ e IOGAativ $(55,0 \pm 3,6$ bpm) em relação ao CTativ (63,2 \pm 5,5 bpm). Os indivíduos do grupo IOGAativ e CTativ são praticantes regulares de exercícios físicos e a maioria dos participantes dos grupos IOGA e CT também o fazem. Sabe-se que o exercício físico regular causa a diminuição da freqüência cardíaca de repouso (FURLAN, PIAZZA, DELL'ORTO, GENTILE, CERUTTI, PAGANI \& MALLIANI, 1993). A diminuição da FC em praticantes de ioga, após um período regular de 6 a 12 semanas de prática de ioga, foi também observada em outros estudos (BOWMAN et al, 1997; DAMODARAN et al, 2002 e JOSEPH et al, 1981). UDUPA, SINGH e SETTIWAR, 1975 não encontraram diferença significativa na $\mathrm{FC}$ em praticantes de ioga após 24 semanas de prática.

O IP foi maior no grupo IOGA $(1036,0 \pm 111,2 \mathrm{~ms})$ comparado ao CT $(943,9 \pm 85,3 \mathrm{~ms})$ desconsideradas as fases do protocolo (BASAL ou APÓS A PRÁTICA) e no grupo IOGAativ (1101,6 \pm 72,6 ms) comparado ao CTativ $(957,8 \pm 92,6 \mathrm{~ms})$ quando considerada a condição APÓS A PRÁTICA. Estes 
dados sugerem que é possível haver uma influência da prática na modulação autonômica sobre o IP. O DIP foi maior no grupo IOGA $(68,8 \pm 22,8 \mathrm{~ms})$ comparado ao CT $(51,1 \pm 21,7 \mathrm{~ms})$ e IOGAativ $(77,8 \pm 14,1 \mathrm{~ms})$ comparado ao CTativ (41,5 $\pm 17,8 \mathrm{~ms})$. A diminuição da variabilidade do intervalo de pulso está associada a um aumento da mortalidade após infarto agudo do miocárdio (KLEIGER, MILLER, BIGGER \& MOSS, 1987).

A MBF foi maior no grupo IOGAativ comparado ao CTativ $(2157,3 \pm$ 1159,4 e $876,0 \pm 989,4 \mathrm{~ms}^{2}$ respectivamente). O significado fisiológico desta faixa de freqüência ainda é pouco conhecido.

A BF normalmente correlacionada com a modulação autonômica simpática e parassimpática foi maior no grupo IOGAativ comparado ao CTativ $\left(1523,7 \pm 964,6\right.$ e 494,7 $\pm 417,1 \mathrm{~ms}^{2}$ respectivamente). Em relação ao valor BASAL, houve aumento de BF no grupo IOGAativ comparado ao grupo CTativ.

A AF relacionada à modulação autonômica parassimpática e arritmia sinual respiratória foi maior no grupo IOGAativ comparada ao grupo CTativ (1684,4 $\pm 847,9$ e $462,6 \pm 375,1 \mathrm{~ms}^{2}$ respectivamente). Não houve alteração no índice de BF/AF nas condições estudadas.

O respirograma foi avaliado apenas no domínio do tempo e sem avaliação da duração dos ciclos de inspiração e expiração. Isto impede extrair da análise informações acerca de possíveis interações entre o sistema cardiovascular e respiratório ou mecanismos de controle central. Neste estudo, os praticantes e não praticantes de ioga foram instruídos a respirar livremente. Esta estratégia traz a vantagem de não introduzir um fator de stress para os participantes do protocolo, por outro lado, há a possibilidade de que oscilações da freqüência respiratória aumentem componentes de faixas do espectro do IP, dificultando a identificação de origem das mesmas (BROWN, BEIGHTOL, 
KOH \& ECKBERG, 1993; PITZALIS, MASTROPASQUA, MASSARI, PASSANTINO, COLOMBO, MANNARINI, FORLEO \& RIZZON, 1998).

Não houve diferença na freqüência respiratória entre os grupos estudados, diferentemente de LEITE, 1999 que relata valores menores da FR em praticantes de ioga em relação aos não praticantes de ioga. Também não foi observada alteração na FR após a prática dos asanas, diferentemente de DAMODARAN, 2002 que relata valores menores da FR após 12 semanas de prática de ioga.

RAJU, PRASAD, VENKATA, MURTHY e REDDY (1997) identificaram, através de teste em esteira, diminuição do volume minuto, freqüência cardíaca, consumo de oxigênio e quociente respiratório em seis professoras de educação física após quatro semanas de prática de ioga (três horas diárias) compreendendo exercícios respiratórios e asanas.

SANTAELLA (2003) verificou maior redução da pressão arterial em indivíduos normotensos e hipertensos quando combinados exercício físico aeróbio e a prática de relaxamento da ioga em comparação com a prática isolada de exercício físico ou relaxamento.

Em nosso estudo, quando comparamos os resultados obtidos na condição basal entre os grupos praticantes de atividade física e praticantes de atividade física mais ioga, observou-se que este último apresentou menor FC, maior PTOT e maior variância do intervalo de pulso. Estes resultados sugerem que a prática de ioga pode ser benéfica também para indivíduos com atividade física regular.

A diminuição mais acentuada da FC e aumento no DFC, IP, DIP, PTOT, VAR e na potência espectral nas faixas de MBF, BF e AF no grupo praticante de ioga e atividade física regulares em relação ao grupo praticante de 
atividade física somente, sugerem que a prática da ioga possa resultar numa melhor resposta adaptativa do organismo.

Em resumo, o estudo mostrou que os praticantes de ioga apresentaram FC menor e alterações nos parâmetros estudados que sugerem efeitos cumulativos e sinérgicos da prática de asanas e outras atividades físicas moderadas. 


\section{REFERÊNCIAS}

AKSELROD, S., GORDON, D.,UBEL, F.A., SHANNON, D.C., BARGER, A.C., COHEN, R.J. Power spectral analysis of heart rate fluctuation: a quantitative probe of beat-to-beat cardiovascular control. Science, Washington, v.213, p.219-222, 1981.

ANAND, B.K.; CHHINA, G.S. Investigations on yogis claiming to stop their heart beats. The Indian Journal of Medical Research, New Delhi, v.49, n.1, p.90-94, 1961.

ANAND, B.K.; CHHINA, G.S; SINGH, B. Studies on Shri Ramanand yogi during his stay in an air tight box. The Indian Journal of Medical Research, New Delhi, v.49, n.1, p.82-89, 1961.

ANTELMI, I.; DE PAULA, R.S.; SHINZATO A.R.; PERES C.A.; MANSUR A.J.; GRUPI, C.J. Influence of age, gender, body mass index, and functional capacity on heart rate variability in a cohort of subjects without heart disease. The American journal of cardiology, United States, v.93, n.1, p.381-385, 2004.

BERNARDI, L.; PASSINO, C.; WILMERDING, V.; DALLAM, G.M.; PARKER, D.L.; ROBERT A.R.; APPENZELLER O. Breathing patterns and cardiovascular autonomic modulation during hypoxia induced by simulated altitude. Journal of Hypertension, London, v.19, p.947-958, 2001. 
BERNARDI, L.; SPADACINI, G.; BELWON, J.; HAJRIC, R.; ROSKAMM, H.; FREY, A.W. Effect of breathing rate on oxygen saturation and exercise performance in chronic heart failure. The Lancet, Boston, v. 351, 2, p.13081311, 1998.

BHOLE, M.V.; KARAMBELKAR. Pulse control and yoga practices. YogaMimansa, Lonavla, v.15, n.1, p.39-42, 1972.

BOWMAN, A.J.; CLAYTON, R.H.; MURRAY, A.; REED, J.W.; SUBHAN, M.M.F.; FORD, G.A. Effects of aerobic exercise training and yoga on the baroreflex in health eldery persons. European Journal of Clinical Investigation, Berlin, v.27, p.443-449, 1997.

BROWN, T.E.; BEIGHTOL, L.A.; KOH, J.; ECKBERG, D.L. Important influence of respiration on human R-R interval power spectra is largely ignored. Journal of Applied Physiology, Washington, v.75, n.5, p.2310-2317, 1993.

DAMODARAN, A.; MALATHI, A.; PATIL, N.; SHAH, N.; SURYAVANSHI; MARATHE, S. Therapeutic potential of yoga practices in modifying cardiovascular risk profile in middle aged men and women. The Journal of the Association of Physicians India, Mumbai, v.50, p. 633-640, 2002.

DATEY, K.K.; DESHMUK, S.N.; DALVI, C.P.; VINEKAR, S.L.”Shavasan": a yogic exercise in the management of hypertension. Angiology, New York, v.20, p.325-333, 1969. 
DGAMBARJI, S.; GHAROTE, M.L. Hathapradipika of Svatmarama. Lonavla: Kaivalyadhama S.M.Y.M. Samiti, 1970.

. Gheranda Samhita. Lonavla: Kaivalyadhama S.M.Y.M. Samiti, 1978.

DGAMBARJI, S.; SAHAY, M. Yoga Kosa. Lonavla: Kaivalyadhama S.M.Y.M. Samiti, p. 62, 1991.

Di RIENZO, M.; MANCIA, G.; PARATI, G.; PEDOTTI, A.; ZANCHETTI, A. Blood pressure and heart rate variability: computer analysis, methodology and clinical applications. Amsterdam: IOS Press, 1993. v.4.

ELIADE, M. Yoga inmortalidad y libertad. Buenos Aires: Editorial La Pleyade, 1954.

FLANAGAN, P.; ECCLES, R. Spontaneous changes of unilateral nasal airflow in man. A re-examination of the "nasal cycle". Acta-Otolaryngologica Stockholm, v.117, p.590-595, 1997.

FOX, E.; SHOTTON, K.; ULRICH, C. User manual Sigma Stat 32 v 2.0. San Rafael: Jandel Scientific Software, 1995.

FURLAN, R.; PIAZZA, S.; DELL'ORTO, S.; GENTILE, E.; CERUTTI, S.; PAGANI, M.; MALLIANI, A. Early and late effects of exercise and athletic training on neural mechanisms controlling heart rate. Cardiovascular Research, London, v.27, p.482-488, 1993. 
GANONG, W.F. Review of medical physiology. 3.ed. Connecticut: Appleton \& Lange, 1993.

GOPAL, K.S; ANANTHARAMN, V.; NISHITH, S.D.; BHATNAGAR O.P. The effect of yogasanas on muscular tone and cardio-respiratory adjustments. The Indian Journal of Medical Sciences, Mumbai, v.28, n.10, p.438-443, 1974.

JOSEPH, S.; SRIDHARAN, K.; PATIL, S.K.B.; KUMARIA, M.L.; SELVAMURTY, W.; JOSEPH, N.T.; NAYAR, H.S. Study of some physiological and biochemical parameters in subjects undergoing yogic training. The Indian Journal of Medical Research, New Delhi, v.74, p.120-124, 1981.

KARAMBELKAR, P.V. Patanjali's yoga sutras. Mumbai: Kaivalyadhama, 1986.

KLEIGER, JUNIOR, R.E.; MILLER, J.P.; BIGGER, J.T.; MOSS, A.J.; MULTICENTER POST-INFARCTION RESEARCH GROUP. Decreased heart rate variability and its association with increased mortality after acute myocardial infarction. The American Journal of Cardiology, New York, v.59, p.256-262, 1987.

KUVALAYANANDA, S. Asanas. Tradução de Ignez Novaes Romeu. São Paulo: Cultrix, 1993. 
KUVALAYANANDA, S.; VINEKAR, S.L. Yogic therapy. New Delhi: Central Health Education Bureau / Ministry of Health, Government of Índia Press, 1971.

LEAL, A.R.M. A variabilidade da freqüência cardíaca em praticantes experientes de Swasthya Yôga, durante a prática de meditação, relaxação e três exercícios-tipo de Pránáyáma. 1996. 86f. Tese (Mestrado) - Faculdade de Medicina, Universidade do Porto, Porto.

LEHRER, P.; SASAKI, Y.; SAITO, Y. Zazen and cardiac variability. Psychosomatic Medicine, Baltimore, v.61, p.812-821, 1999.

LEITE M.R.R. Estudo dos padrões do movimento respiratório e do comportamento cardiovascular em mulheres idosas praticantes de yoga. 1999. 82 f. Tese (Mestrado) - Faculdade de Educação Física, Universidade Estadual de Campinas, Campinas.

MALLIANI, A.; LOMBARDI, F.; PAGANI, M. Power spectrum analyasis of heart rate variability: a tool to explore neural regulatory mechanisms. British Heart Journal, London, v.1, n.71, p.1-2, 1994.

MALLIANI A.; PAGANI M., LOMBARDI F., CERUTTI, S. Cardiovascular neural regulation explored in the frequency domain. Circulation, Dallas, v.84, n.2, p. 482-492, 1991.

MANCHANDA S.C.; NARANG, R. Yoga and coronary artery disease. Indian Heart Journal, New Delhi, v.50, p.227-228, 1998. 
PAGANI, M.; LOMBARDI, F.; GUZZETTI S.; RIMOLDI, O.; FURLAN, R.; PIZZINELLI, P.; SANDRONE，G.; MALFATTTO, G.; DELL'ORTO, S.; PICCALUGA, E.; TURIEL, M.; BASELLI, G.; CERUTTI, S.; MALLIANI, A. Power spectral analysis of heart rate and arterial pressure variabilities as a marker of sympatho-vagal interaction in man and conscious dog. Circulation Research, Baltimore, v.59, p.178-193, 1986.

PARATI, G.; SAUL, J.P.; DI RIENZO, M.; MANCIA, G. Spectral analysis of blood pressure and heart rate variability in evaluating cardiovascular regulation. A critical appraisal. Hypertension, Dallas, v.25; p. 1276-1286, 1995.

PATEL, C. Yoga and biofeedback in the management of hypertension. The Lancet, Boston, v.10, p.1053-1055, 1973.

. 12-month follow-up of yoga and bio-feedback in the management of hypertension. The Lancet, Boston, v.11, p.62-64, 1975.

PATEL, C.; NORTH, W.R.S. Randomised controlled trial of yoga and biofeedback in management of hypertension, The Lancet, Boston, v.19, p.93-95, 1975.

PENG, C.-K.; HENRY, I.C.; MIETUS, J.E.; HAUSDORFF, J.M.; KHALSA, G.; BENSON, H.; GOLDBERGER, A.L. Heart rate dynamics during three forms of meditation. International Journal of Cardiology, Amsterdam, v.95, p.19-27, 2004. 
PITZALIS, M.V; MASTROPASQUA, F.; MASSARI, F.; PASSANTINO, A.; COLOMBO, R.; MANNARINI, A.; FORLEO, C.; RIZZON, P. Effect of respiratory rate on the relationships between RR interval and systolic blood pressure fluctuations: a frequency-dependent phenomenon. Cardiovascular Research, London, v.38, p.332-339, 1998.

RAJU, P.S.; PRASAD, K.V, VENKATA, R.Y; MURTHY, K.J, REDDY, M.V Influence of intensive yoga training on physiological changes in six adult womem: a case report. Journal of Alternative and Complementary Medicine, New York, v.3, n.3, p.291-5, 1997.

ROMPELMAN, O.; SNIJDERS, J.B.I.M.; SPRONSEN, C.J.V. The measurement of heart rate variability spectra with the help of a personal computer. IEEE Transactions on biomedical engeneering, New York, v 29, n.7, 1982.

SANTAELLA, D.F. Efeitos do relaxamento e do exercício físico nas respostas pressórica e autonômica em indivíduos normotensos e hipertensos. 2003. 215f. Tese (Mestrado) - Faculdade de Medicina, Universidade de São Paulo, São Paulo.

SLEIGHT P.; CASADEI, B. Relationships between heart rate, respiration and blood pressure variabilities. In: HEART rate variability. New York: Futura, 1995. Cap.24, p.311-327. 
SOUZA, E.D.P. O uso do yoga como procedimento terapêutico complementar na assistência ao cliente neurótico. 1999. 117 f. Tese (Mestrado) - Escola de enfermagem de Ribeirão Preto, Universidade de São Paulo, Ribeirão Preto.

SOVIK, R. The science of breathing: the yogic view. Progress in Brain Research, Amsterdam, v.122, p.491-505, 2000.

SPICUZZA, L.; GABUTTI, A.; PORTA, C.; MONTANO N.; BERNARDI L. Yoga and chemoreflex response to hypoxia and hypercapnia. The Lancet, Boston, v.356, n.28, 2000.

STANESCU, D.C; NEMERY, B.; VERITER, C.; MARECHAL, C. Pattern of breathing and ventilatory response to $\mathrm{CO} 2$ in subjects practicing hatha-yoga. Journal of Applied Physiology, Washington, v.51, n.6, p.1625-1629, 1981.

SUNDAR S.; AGRAWAL, S.K.; SINGH, V.P.; BHATTACHARYA, K.N.; UDUPA K.N.; VAISH, S.K. Role of yoga in management of essential hypertension. Acta Cardiologica, Bruxelles, v.39, n.3, p.203-208, 1984.

TAIMNI, I.K. A ciência do yoga. Tradução Milton Lavrador. Brasília: Teosófica, 1996.

TASK FORCE OF THE EUROPEAN SOCIETY OF CARDIOLOGY THE NORTH AMERICAN SOCIETY OF PACING ELECTTTROPHYSIOLOGY. Heart rate variability: standards of measurement, physiological interpretation, and clinical use. Circulation, Dallas, v.93, p.1043-1065, 1996. 
UDUPA, K.N.; SINGH, R.H. The scientific basis of yoga. The Journal of the American Medical Association, Chicago, v.220, n.10, 1972.

UDUPA, K.N.; SINGH, R.H.; SETTIWAR R.M. Physiological and biochemical studies on the effect of yogic and certain other exercises. The Indian Journal of Medical Research, New Delhi, v.63, n.4, 1975.

UDUPA, K.N.; SINGH, R.H.; YADAV, R.A. Certain studies on psychological and biochemical response to the practice of hatha yoga in young normal volunteers. The Indian Journal of Medical Research, New Delhi, v.61, n.2, 1973.

USHIZIMA, M.R. Desenvolvimento de ferramentas para análise de sinais biológicos nos domínios do tempo, freqüência e tempo-freqüência: aplicação ao estudo da regulação da pressão arterial. 2000. 152 f. Tese (Doutorado) - Faculdade de Engenharia Elétrica e de Computação, Universidade Estadual de Campinas, Campinas.

WENGER, M.A.; BAGCHI B.K.; ANAND, B.K. Experiments in India on "voluntary" control of the heart and pulse. Circulation, Dallas, v. 24, p.1319$1325,1961$. 


\section{ANEXO I - Questionário para registro de voluntários}

Registro de voluntários para estudo sobre efeitos da prática de Ioga

Nome:

Data de nascimento:

/ 1

$\begin{array}{ll}\text { Peso: } & \text { Altura: }\end{array}$

masculino $\square \quad$ feminino $\square$

Profissão atual (no caso de 02 profissões indicar a atividade principal):

Pratica ioga regularmente:

Quantos dias por semana?

Duração média da prática:

$$
\operatorname{sim} \square \quad \text { não } \square
$$

$1 \square \quad 2 \square 3 \square 4 \square 5 \square 6 \square 7 \square$

10 min. $\square 15$ min. $\square 20$ min. $\square 30$ min. $\square 1 \mathrm{~h} \square$

Pratica regularmente

\begin{tabular}{|l|l|l|}
\hline Brahma Mudra & Sim $\square$ Não $\square$ \\
\hline
\end{tabular}


ANEXO I - Questionário para registro de voluntários (cont.)

\begin{tabular}{|l|l|l|}
\hline Vakrasana & Sim $\square \quad$ Não $\square$ \\
\hline Chakrasana & Sim $\square \quad$ Não $\square$ \\
\hline Não $\square$ \\
\hline
\end{tabular}

Pratica outras atividades físicas: $\operatorname{Sim} \square \quad$ Não $\square$

Quais?

Caminhada

Corrida

Ciclismo

Natação

Ginástica

Musculação

Dança

Outros:

Quantos dias por semana?

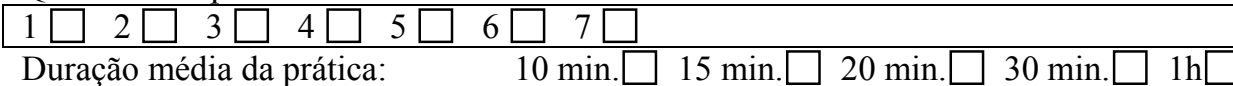


ANEXO I - Questionário para registro de voluntários (cont.)

Faz uso regular de medicamentos? $\quad \operatorname{Sim} \square \quad$ Não $\square$

Observações:

Local e data:

Assinatura: 
ANEXO II - Termo de consentimento livre e esclarecido

\author{
ESCOLA DE EDUCAÇÃO FÍSICA E ESPORTE DA \\ UNIVERSIDADE DE SÃO PAULO \\ TERMO DE CONSENTIMENTO LIVRE E ESCLARECIDO \\ (Instruções para preenchimento no verso)
}

I - DADOS DE IDENTIFICAÇÃO DO SUJEITO DA PESQUISA OU RESPONSÁVEL LEGAL

1. NOME DO INDIVÍDUO

DOCUMENTO DE IDENTIDADE $\mathrm{N}^{\circ}$ :

SEXO : .M F

DATA NASCIMENTO:

ENDEREÇO

$\mathrm{N}^{\mathrm{o}}$

APTO

BAIRRO:

CEP:.

TELEFONE: DDD (...........)

2. RESPONSÁVEL LEGAL:

NATUREZA (grau de parentesco, tutor, curador,etc.)

DOCUMENTO DE IDENTIDADE :....

SEXO: $M \quad F$

DATA NASCIMENTO.: ....../.............

ENDEREÇO:

$\mathrm{N}^{\mathrm{o}}$

APTO:

BAIRRO:

CIDADE:

CEP: TELEFONE: DDD

II - DADOS SOBRE A PESQUISA CIENTÍFICA

1. TÍTULO DO PROJETO DE PESQUISA:

Estudo dos efeitos da prática de ioga nas freqüências cardíaca e respiratória e no equilíbrio.

2. PESQUISADOR RESPONSÁVEL: Prof. Dr. Alberto Carlos Amadio.

3. CARGO/FUNÇÃO: Professor Titular.

4. AVALIAÇÃO DO RISCO DA PESQUISA:

$\begin{array}{lll}\text { RISCO MÍNIMO } & \mathrm{X} & \text { RISCO MÉDIO } \\ \text { RISCO BAIXO } & & \text { RISCO MAIOR }\end{array}$

(probabilidade de que o indivíduo sofra algum dano como conseqüência imediata ou tardia do estudo)

5. DURAÇÃO DA PESQUISA : 1 hora e 30 minutos

III - EXPLICAÇÕES DO PESQUISADOR AO INDIVÍDUO OU SEU REPRESENTANTE LEGAL SOBRE A PESQUISA, CONSIGNANDO:

1.Justificativa e objetivos da pesquisa 


\section{ANEXO II - Termo de consentimento livre e esclarecido (cont.)}

Os objetivos desta pesquisa são identificar e avaliar os efeitos da prática de ioga nas freqüências cardíaca e respiratória e no equilíbrio.

2. Procedimentos que serão utilizados e propósitos, incluindo a identificação dos procedimentos que são experimentais

Chegando ao laboratório, o (a) senhor (a):

a) Responderá um questionário informando dados pessoais e hábitos de atividade física (Registro de voluntários para estudo sobre efeitos da prática de ioga)

b) Eletrodos de superfície (adesivos) serão colocados no tórax após limpeza do local com álcool e uma cinta de pano será colocada em sua cintura. Os aparelhos citados não apresentam qualquer risco para a saúde.

c) Ficará relaxado, deitado de costas durante 20 minutos, para registros.

d) Caso praticante de ioga: realizará uma seqüência de práticas de ioga que incluem: "Brahma mudra", "montanha", "cobra", "torção", "pinça”, "yoga-mudra" e "roda" da forma como está habituado a praticar regularmente.

e) Novo período de relaxamento, deitado de costas durante 20 minutos, para registros.

3. Desconfortos e riscos esperados

Não são esperados desconfortos e riscos desta pesquisa.

4. Benefícios que poderão ser obtidos

Avaliação da prática de ioga por professor de ioga.

5. Procedimentos alternativos que possam ser vantajosos para o indivíduo

Não se aplica.

\section{IV - ESCLARECIMENTOS DADOS PELO PESQUISADOR SOBRE GARANTIAS DO SUJEITO DA PESQUISA:}

1. $\mathrm{O}$ (A) senhor (a) terá acesso, a qualquer tempo, às informações sobre procedimentos, riscos e benefícios relacionados à pesquisa, inclusive para esclarecer eventuais dúvidas.

2. O (A) senhor (a) tem a liberdade a qualquer momento de desistir de sua participação neste estudo sendo necessário somente comunicar sua decisão.

3. Os dados fornecidos e os resultados obtidos nos testes serão mantidos em sigilo, guardadas as devidas identificações e mantida a confidencialidade serão analisados e utilizados única e exclusivamente para fins científicos.

4. Não são esperados riscos ou danos à saúde. 


\title{
ANEXO II - Termo de consentimento livre e esclarecido (cont.)
}

\author{
V - INFORMAÇÕES DE NOMES, ENDEREÇOS E TELEFONES DOS RESPONSÁVEIS PELO \\ ACOMPANHAMENTO DA PESQUISA, PARA CONTATO EM CASO DE INTERCORRÊNCIAS \\ CLÍNICAS E REAÇÕES ADVERSAS.
}

Silvia Martins Meireles

Av. Prof. Mello Moraes, 65.

Telefone: 3091-3184

VI. OBSERVAÇÕES COMPLEMENTARES:
Os procedimentos adotados na pesquisa não são invasivos.

VII - CONSENTIMENTO PÓS-ESCLARECIDO

Declaro que, após convenientemente esclarecido pelo pesquisador e ter entendido o que me foi explicado, consinto em participar do presente Projeto de pesquisa.

São Paulo, de de 20

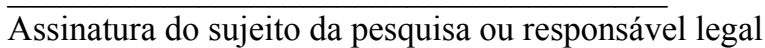

assinatura do pesquisador

(carimbo ou nome legível) 


\section{ANEXO II - Termo de consentimento livre e esclarecido (cont.)}

\section{INSTRUÇÕES PARA PREENCHIMENTO}

(Resolução Conselho Nacional de Saúde 196, de 10 outubro 1996)

1. Este termo conterá o registro das informações que o pesquisador fornecerá ao sujeito da pesquisa, em linguagem clara e accessível, evitando-se vocábulos técnicos não compatíveis com o grau de conhecimento do interlocutor.

2. A avaliação do grau de risco deve ser minuciosa, levando em conta qualquer possibilidade de intervenção e de dano à integridade física do sujeito da pesquisa.

3. O formulário poderá ser preenchido em letra de forma legível, datilografia ou meios eletrônicos.

4. Este termo deverá ser elaborado em duas vias, ficando uma via em poder do paciente ou seu representante legal e outra deverá ser juntada ao prontuário do paciente.

5. A via do Termo de Consentimento Livre e Esclarecido submetida à análise do Comitê de Ética em Pesquisa CEP deverá ser idêntica àquela que será fornecida ao sujeito da pesquisa. 


\section{ANEXO III - Transcrição da gravação que antecede os registros}

(Sinal sonoro) Vamos dar início a nossa sessão de registros, que vai durar 20 minutos.

Primeiramente evite pensar em problemas, compromissos ou se ligar aos ruídos do ambiente. Procure se sentir estável e confortável.

Para isso, se necessário, movimente suas pernas, braços e cabeça até tornar sua posição bem confortável.

Procure deixar suas pernas um pouco afastadas entre si e os braços, um pouco afastados do corpo.

Respire naturalmente, sem controlar o ritmo de sua respiração.

Agora, vamos iniciar um relaxamento.

Feche os olhos, relaxe os pés e os dedos dos pés.

Relaxe as pernas, os joelhos e as coxas.

Passamos às mãos, relaxe os dedos e as mãos.

Relaxe os braços e os ombros.

Relaxe também o quadril e a coluna lombar.

Relaxe as costas, soltando todo o peso do corpo sobre o colchão.

Relaxe o pescoço; a nuca.

Relaxe as bochechas, os lábios e a língua.

Relaxe as orelhas, os olhos, sobrancelhas e a testa.

Relaxe também o couro cabeludo.

Continue relaxado e permaneça assim quando este texto se encerrar até o próximo sinal sonoro.

Procure manter a tranqüilidade e ficar acordado.

(Tempo de duração: 2 min 22 s)

(Sinal sonoro) Lentamente, volte sua atenção para o ambiente e inicie movimentos dos dedos das mãos e dos pés e do restante do corpo.

(Tempo de duração: 12s) 
ANEXO IV - Resultados individuais. Séries temporais, DEP e DEP IP.

Praticante 1 nas condições BASAL e APÓS A PRÁTICA.

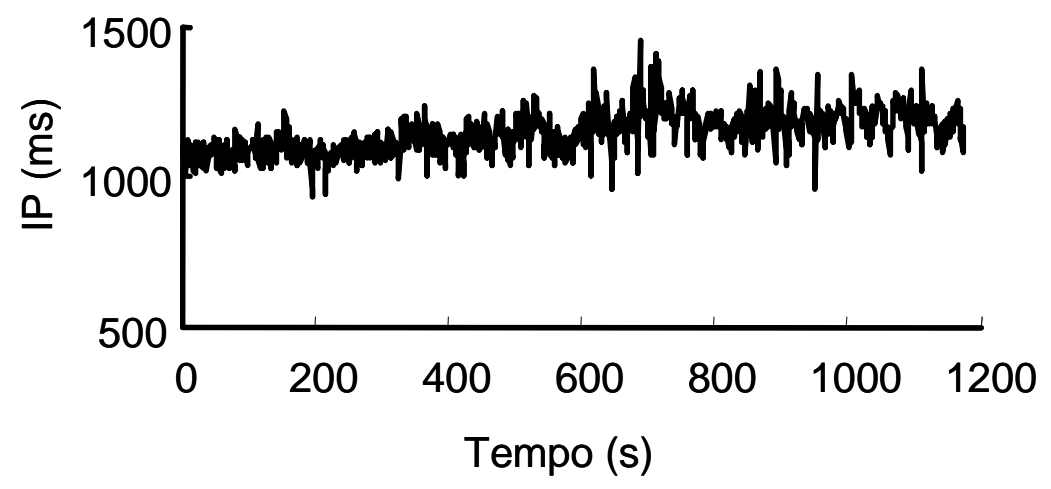

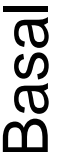
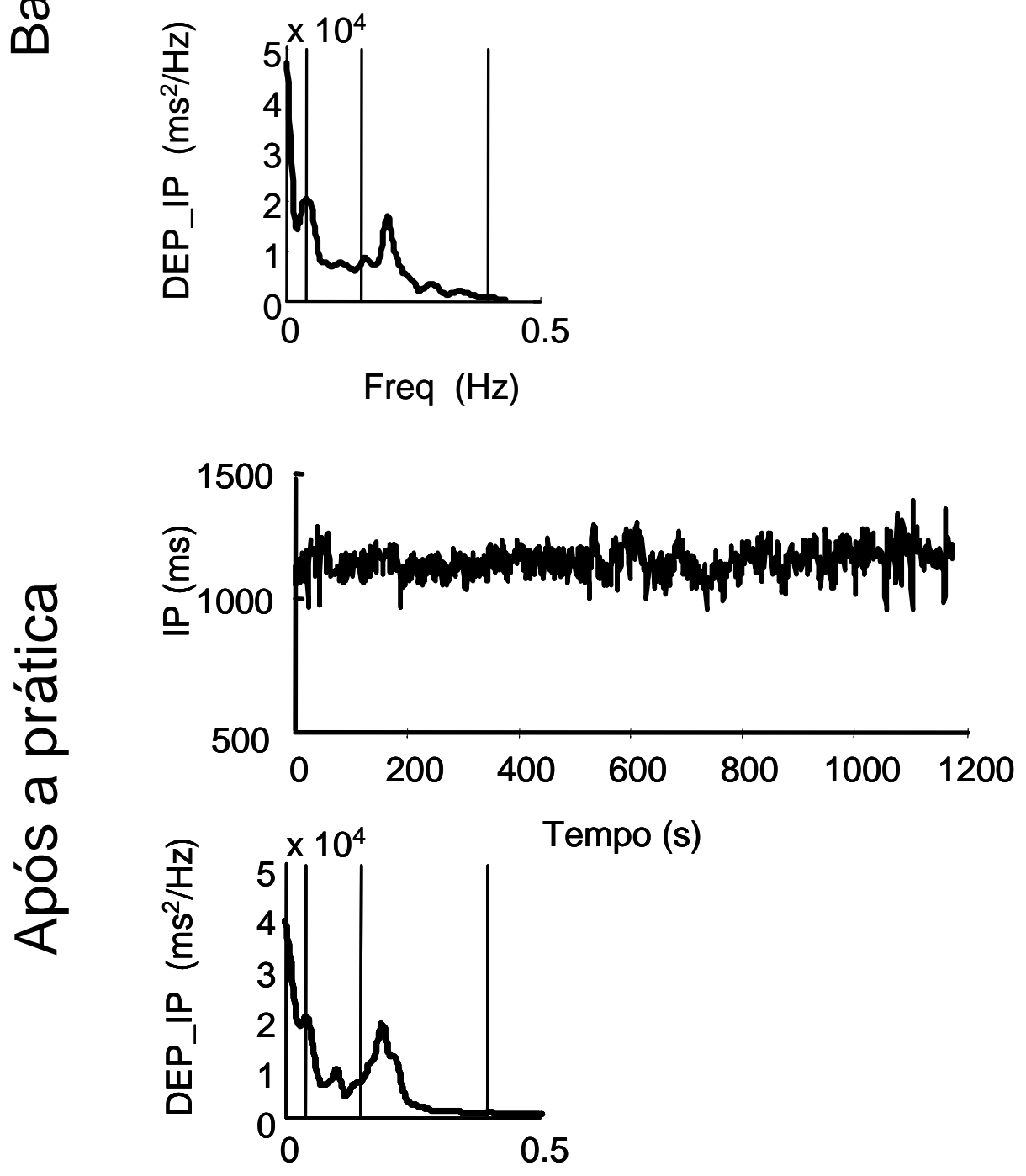
70

ANEXO IV - Resultados individuais. Séries temporais, DEP e DEP IP (cont.) Praticante 2 nas condições BASAL e APÓS A PRÁTICA.
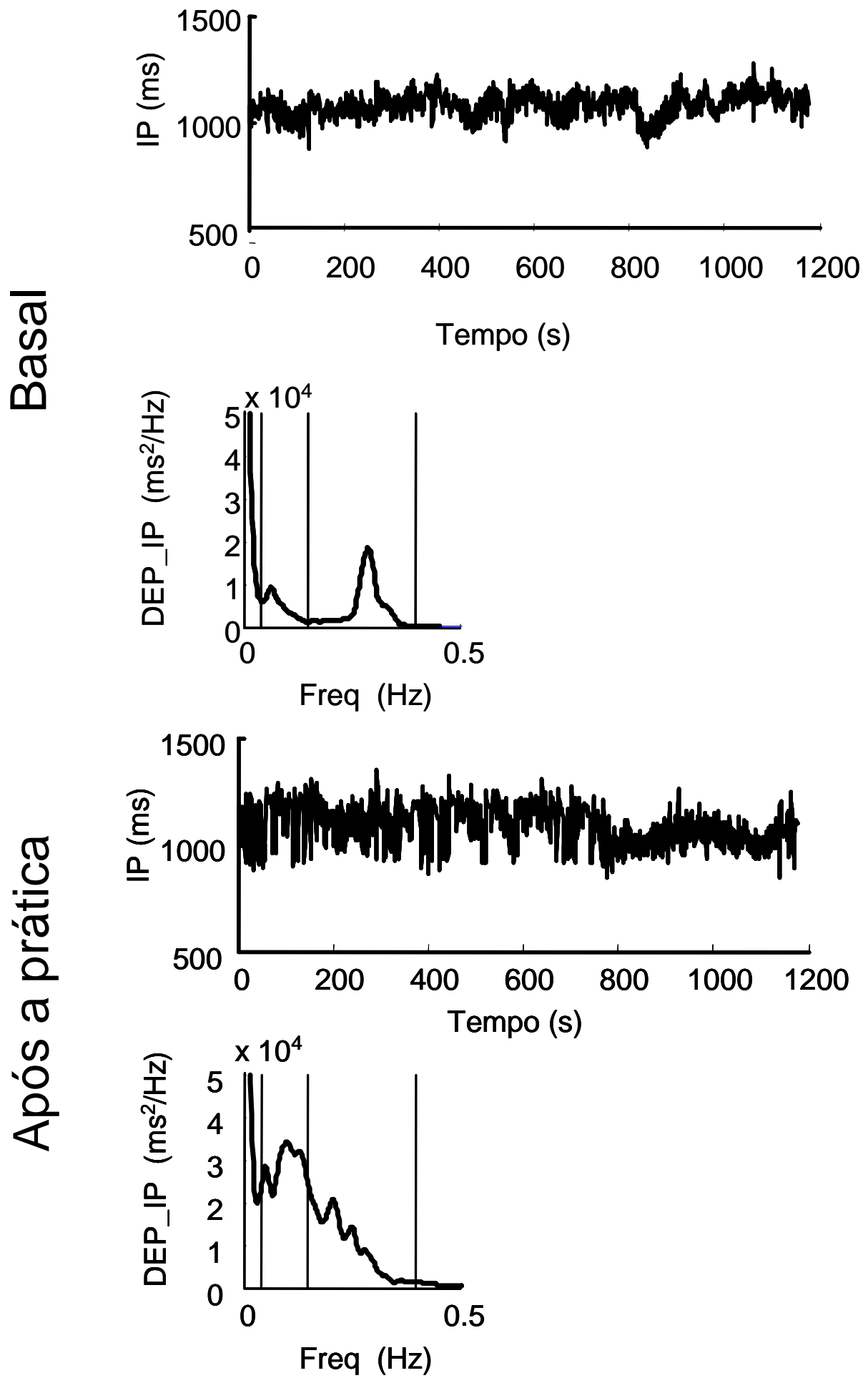
ANEXO IV - Resultados individuais. Séries temporais, DEP e DEP IP (cont.) Praticante 3 nas condições BASAL e APÓS A PRÁTICA.

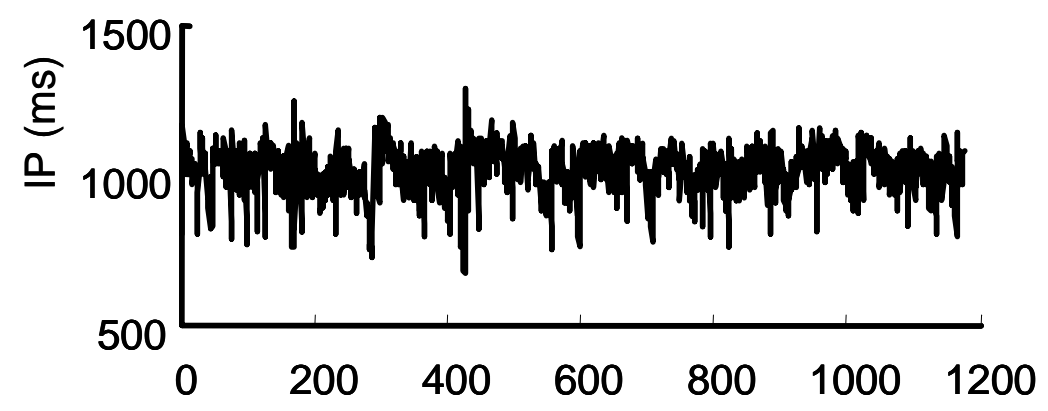

$\bar{\sigma}$
๘
ஸ
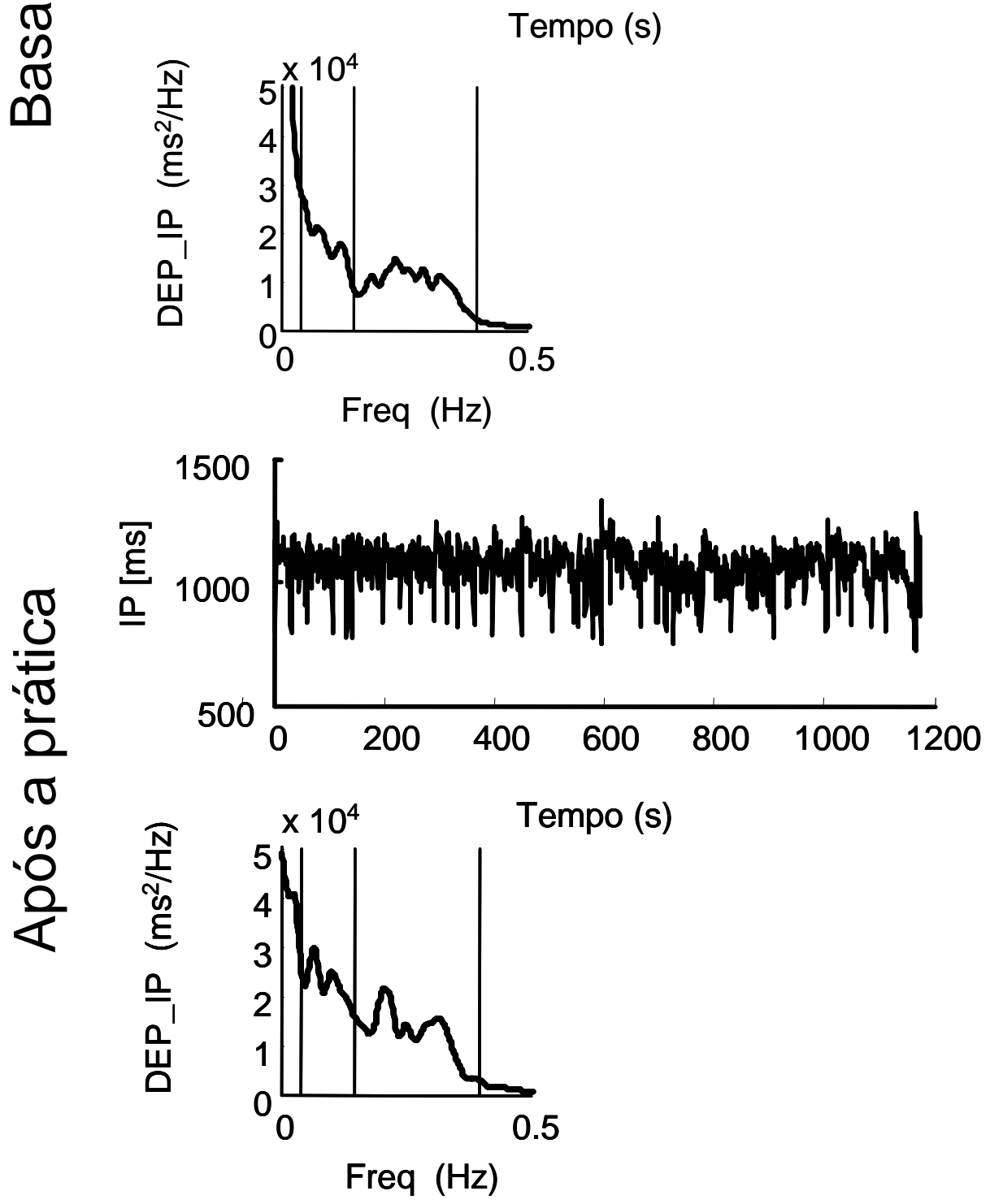
ANEXO IV - Resultados individuais. Séries temporais, DEP e DEP IP (cont.) Praticante 4 nas condições BASAL e APÓS A PRÁTICA.
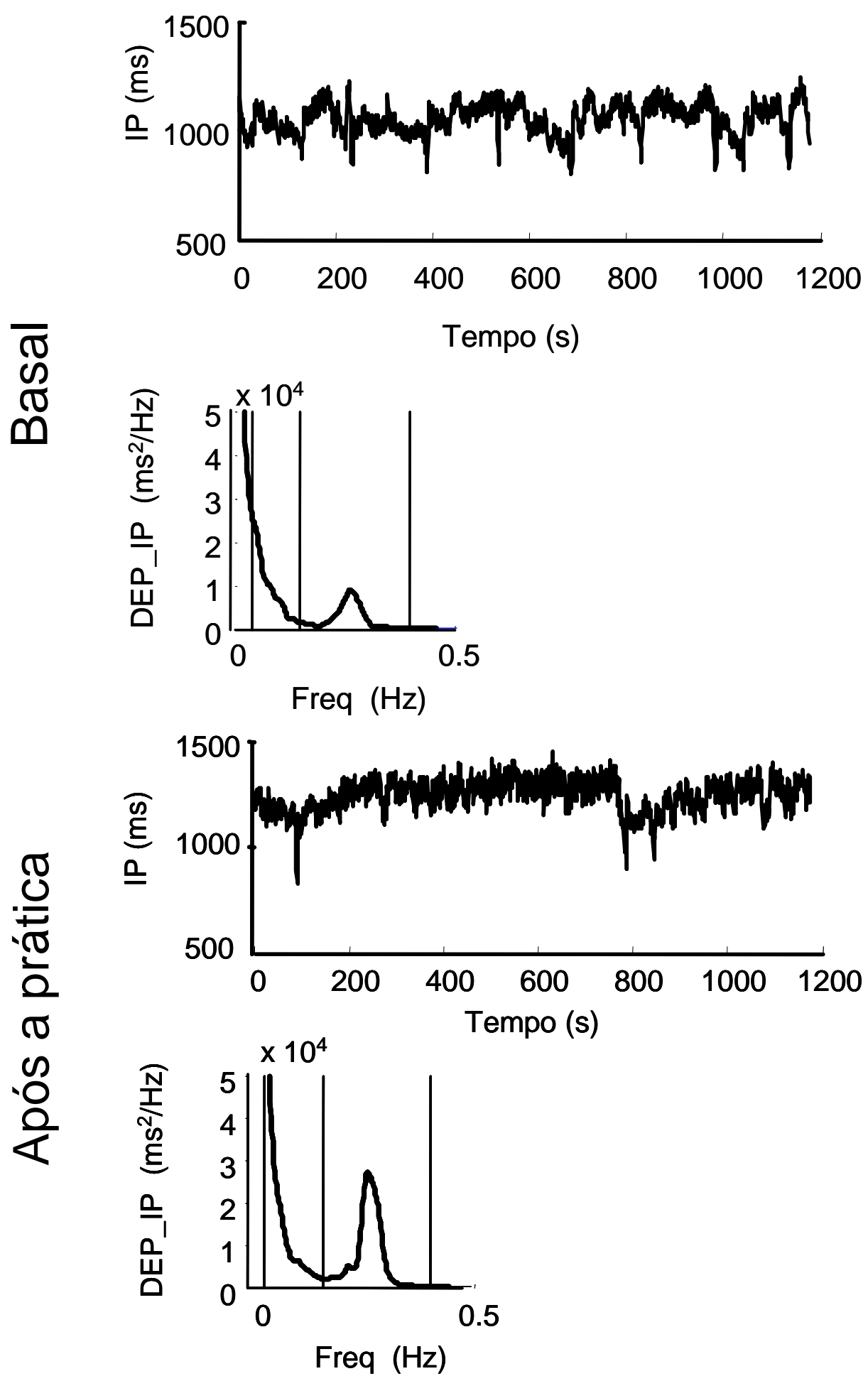
ANEXO IV - Resultados individuais. Séries temporais, DEP e DEP IP (cont.) Praticante 5 nas condições BASAL e APÓS A PRÁTICA.

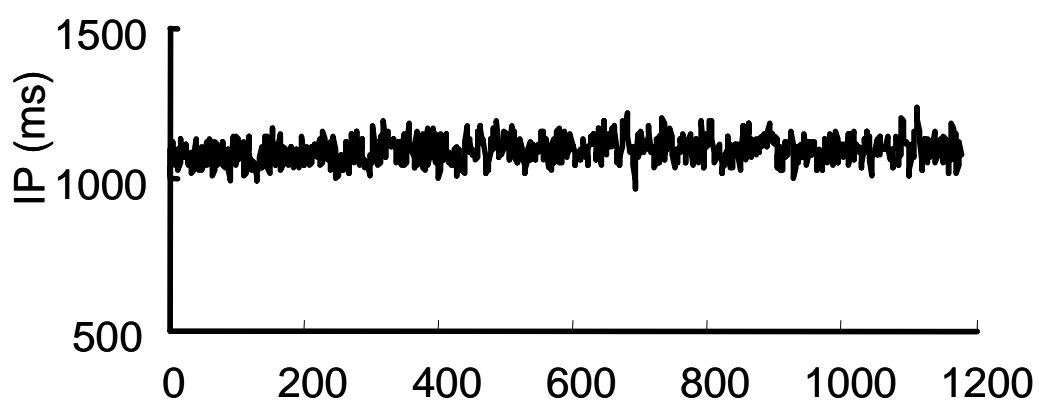

$\bar{\sigma}$
$\tilde{\mathscr{N}}$
ஸ
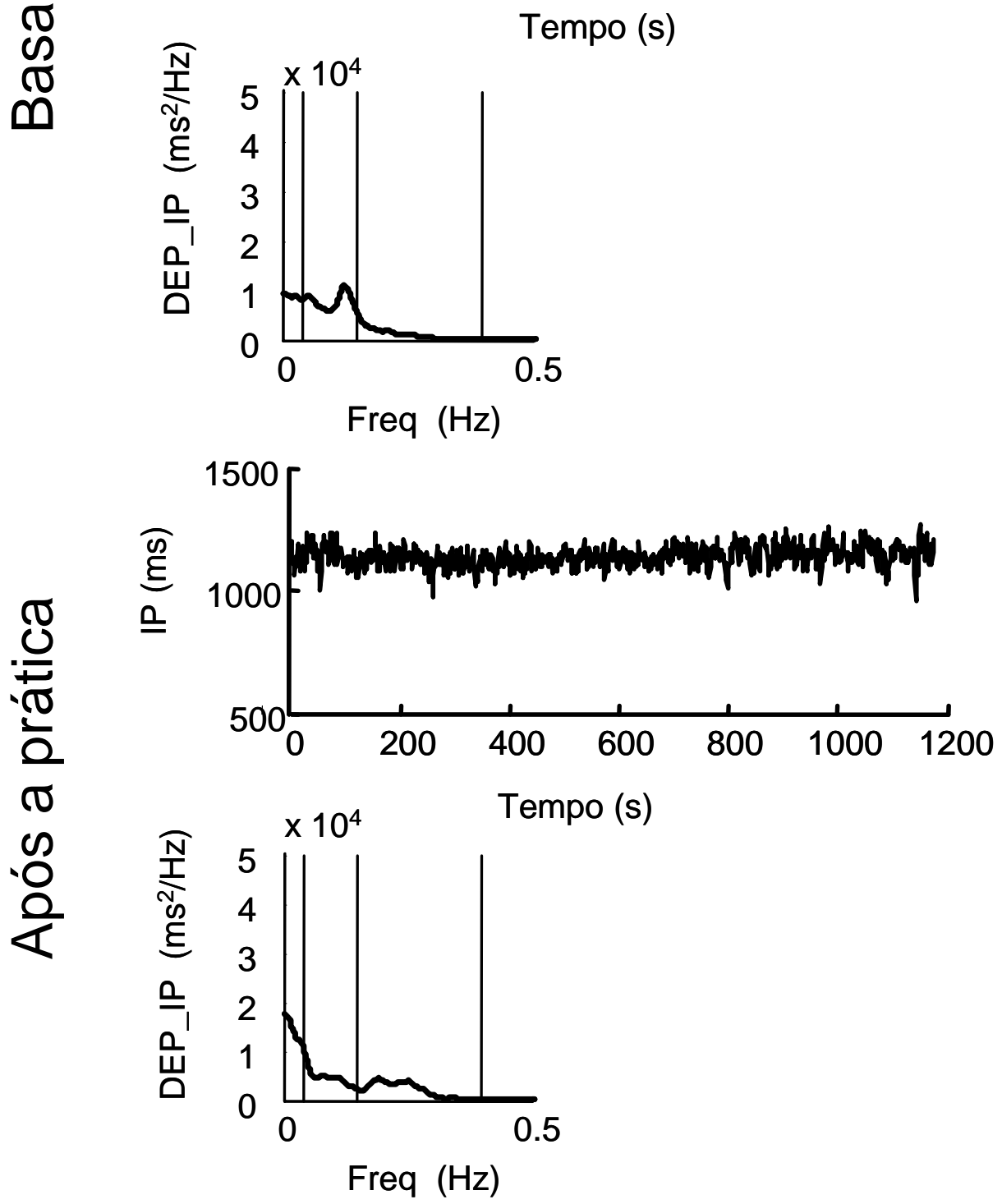
ANEXO IV - Resultados individuais. Séries temporais, DEP e DEP IP (cont.) Praticante 6 nas condições BASAL e APÓS A PRÁTICA.
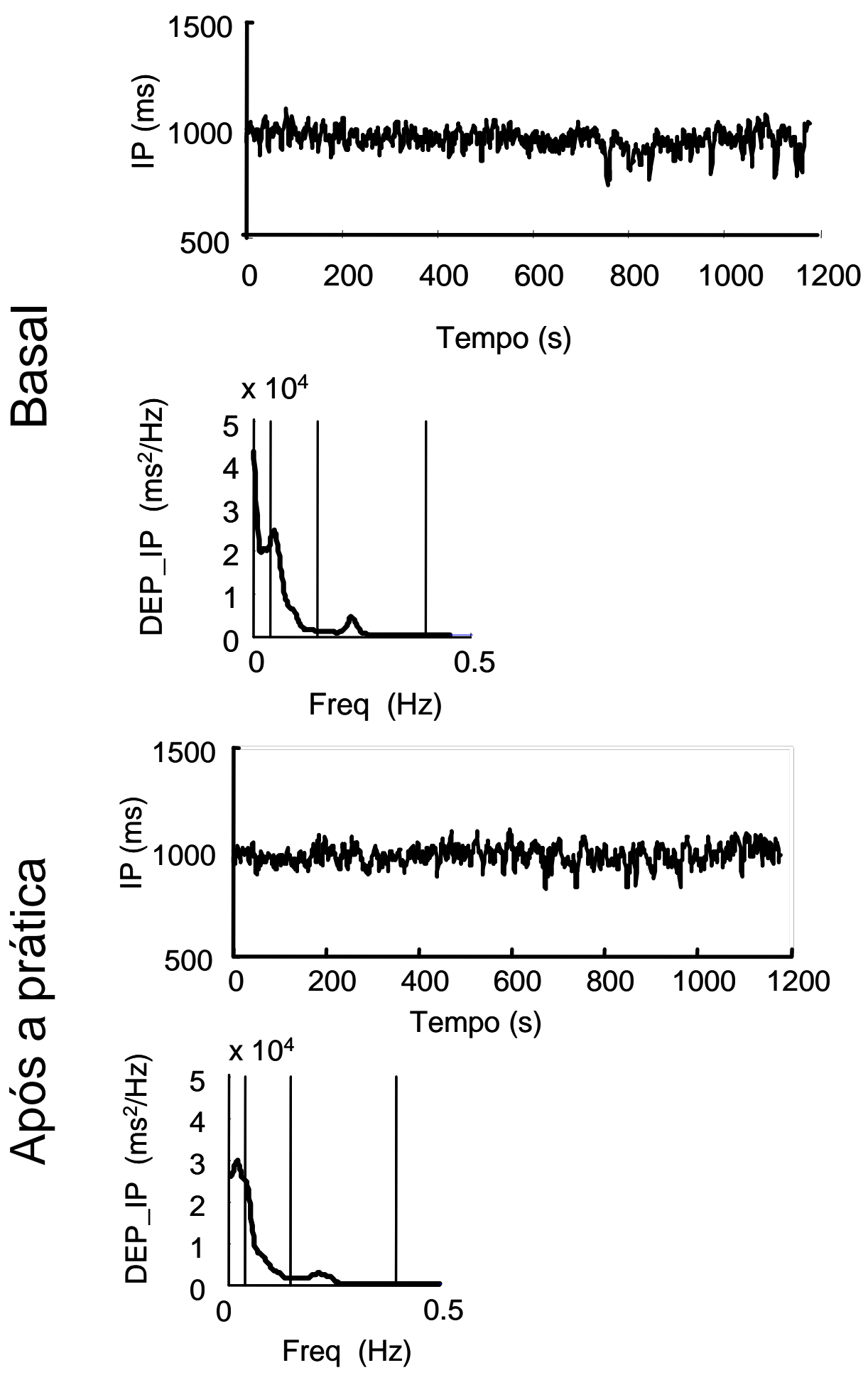
ANEXO IV - Resultados individuais. Séries temporais, DEP e DEP IP (cont.) Praticante 7 nas condições BASAL e APÓS A PRÁTICA.
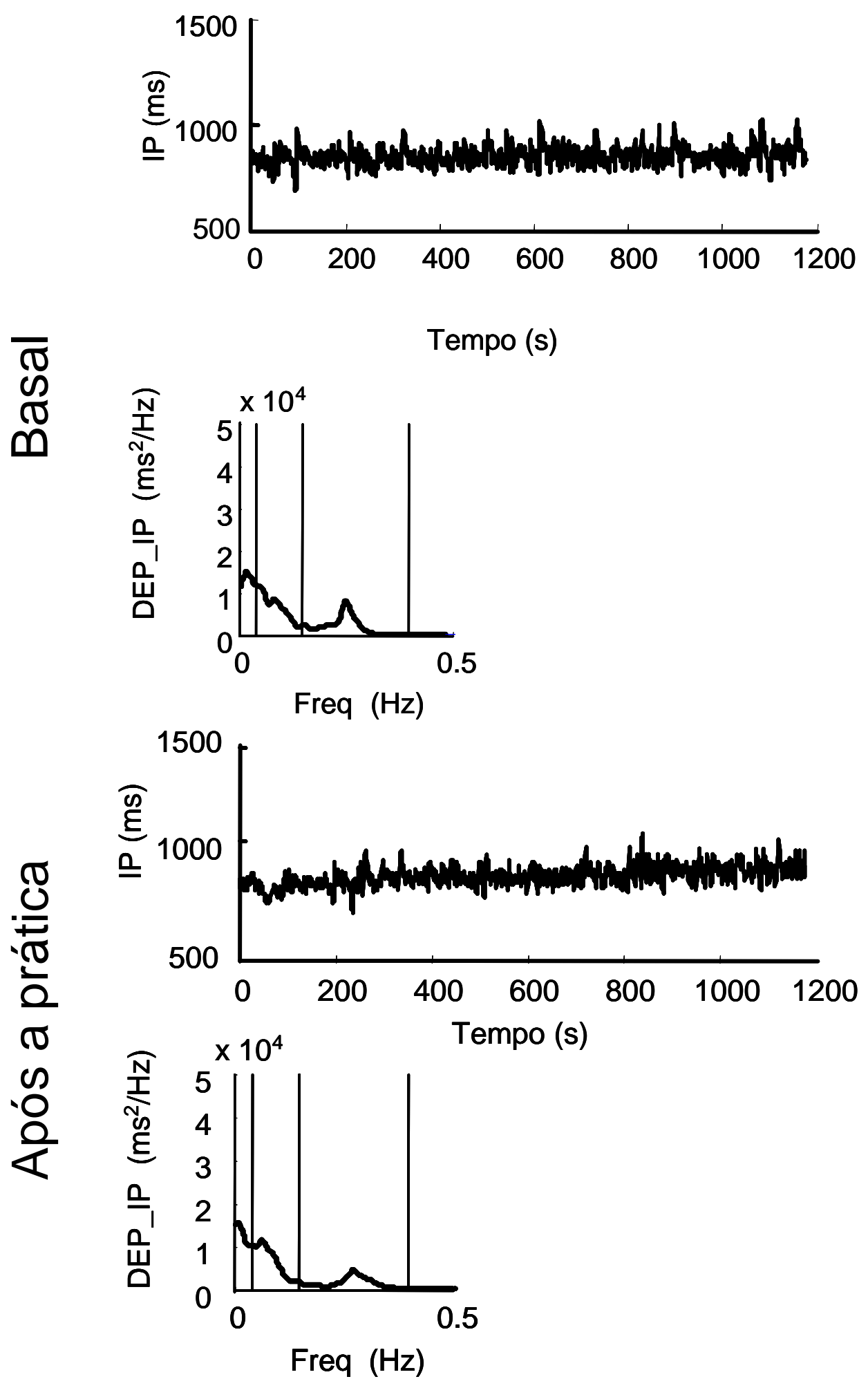
ANEXO IV - Resultados individuais. Séries temporais, DEP e DEP IP (cont.) Praticante 8 nas condições BASAL e APÓS A PRÁTICA.

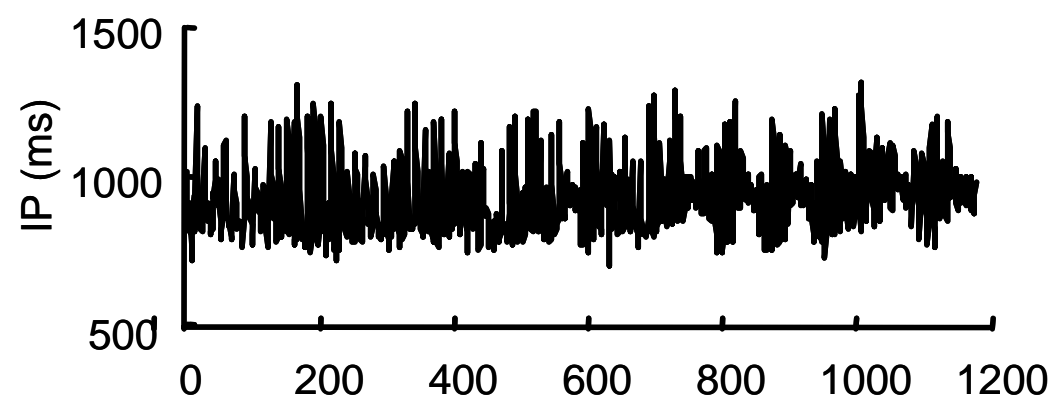

$\bar{\sigma}$
ఠิ
ต
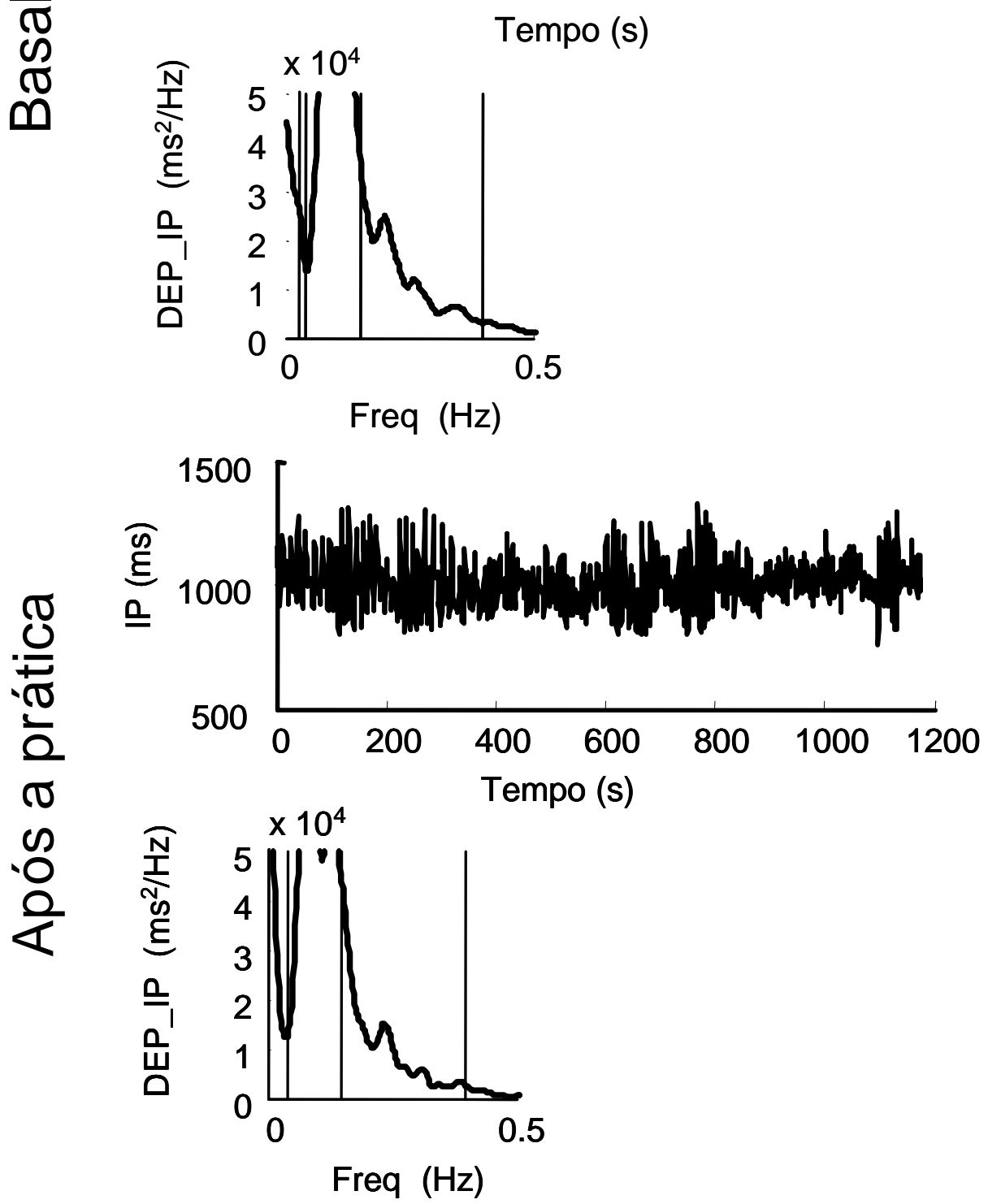
ANEXO IV - Resultados individuais. Séries temporais, DEP e DEP IP (cont.) Controle 1 nas condições BASAL e APÓS A PRÁTICA.

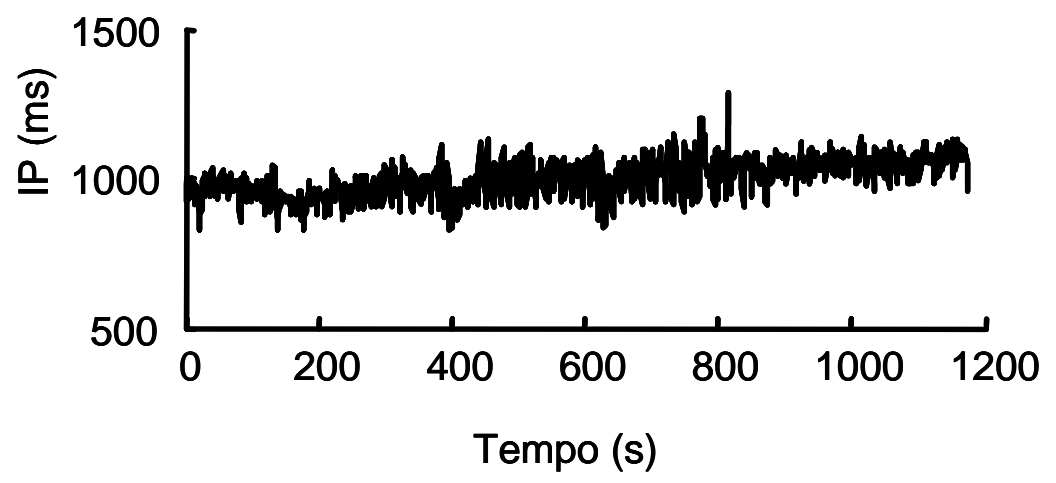

$\bar{\sigma}$

ஸे

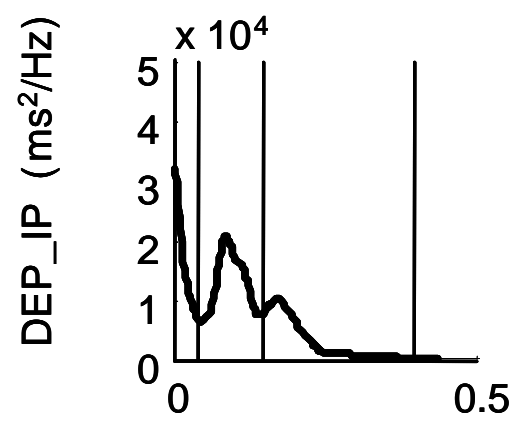

Freq $(\mathrm{Hz})$

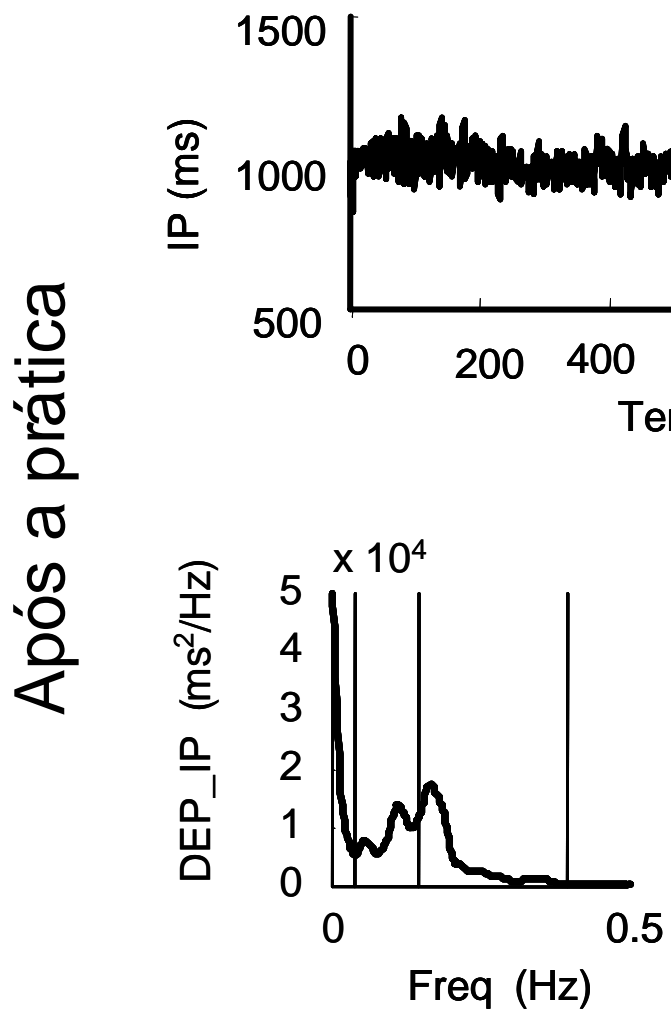


ANEXO IV - Resultados individuais. Séries temporais, DEP e DEP IP (cont.) Controle 2 nas condições BASAL e APÓS A PRÁTICA.

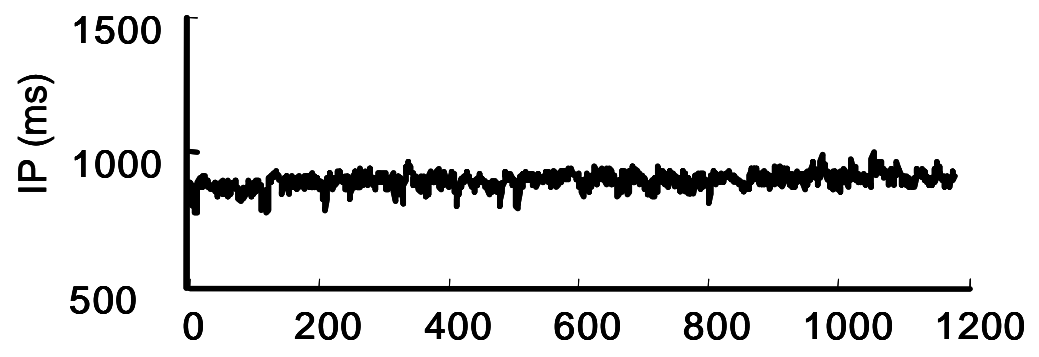

$\bar{\sigma}$
ஸे
ต

Tempo (s)
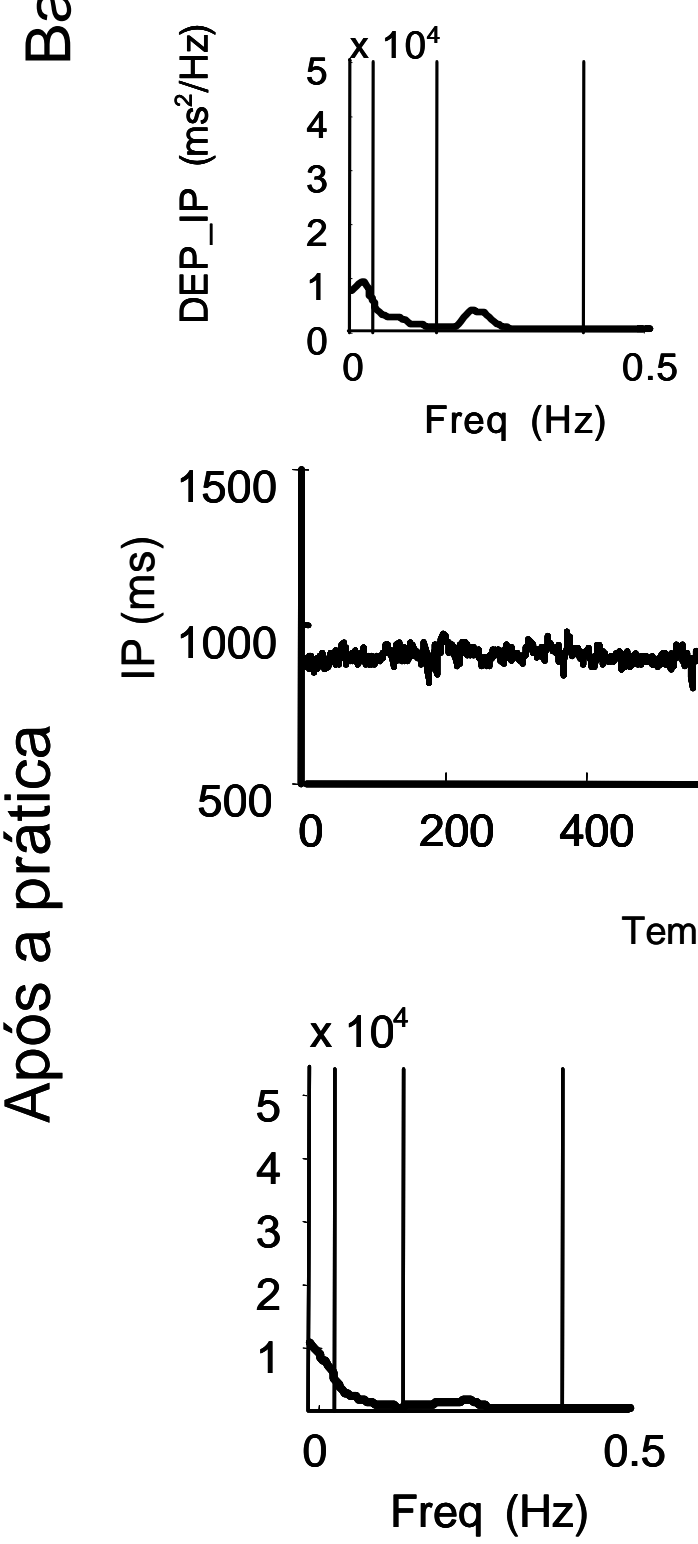
ANEXO IV - Resultados individuais. Séries temporais, DEP e DEP IP (cont.) Controle 3 nas condições BASAL e APÓS A PRÁTICA.

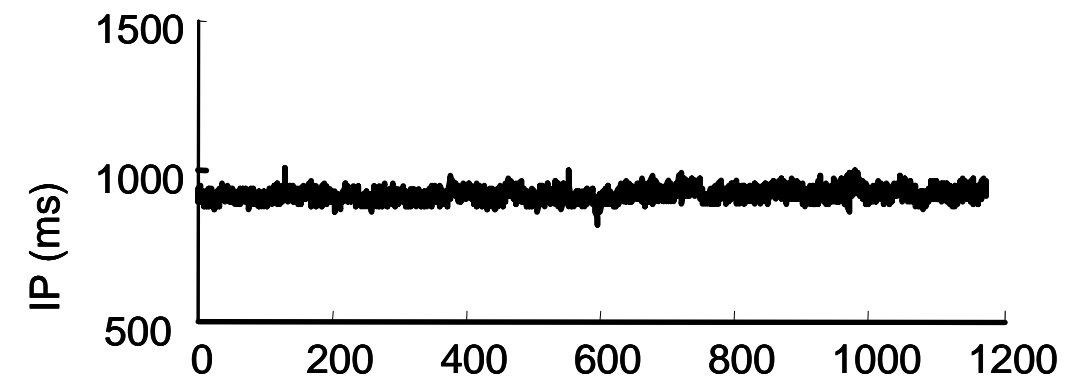

$\bar{\sigma}$

ஸे

Tempo (s)
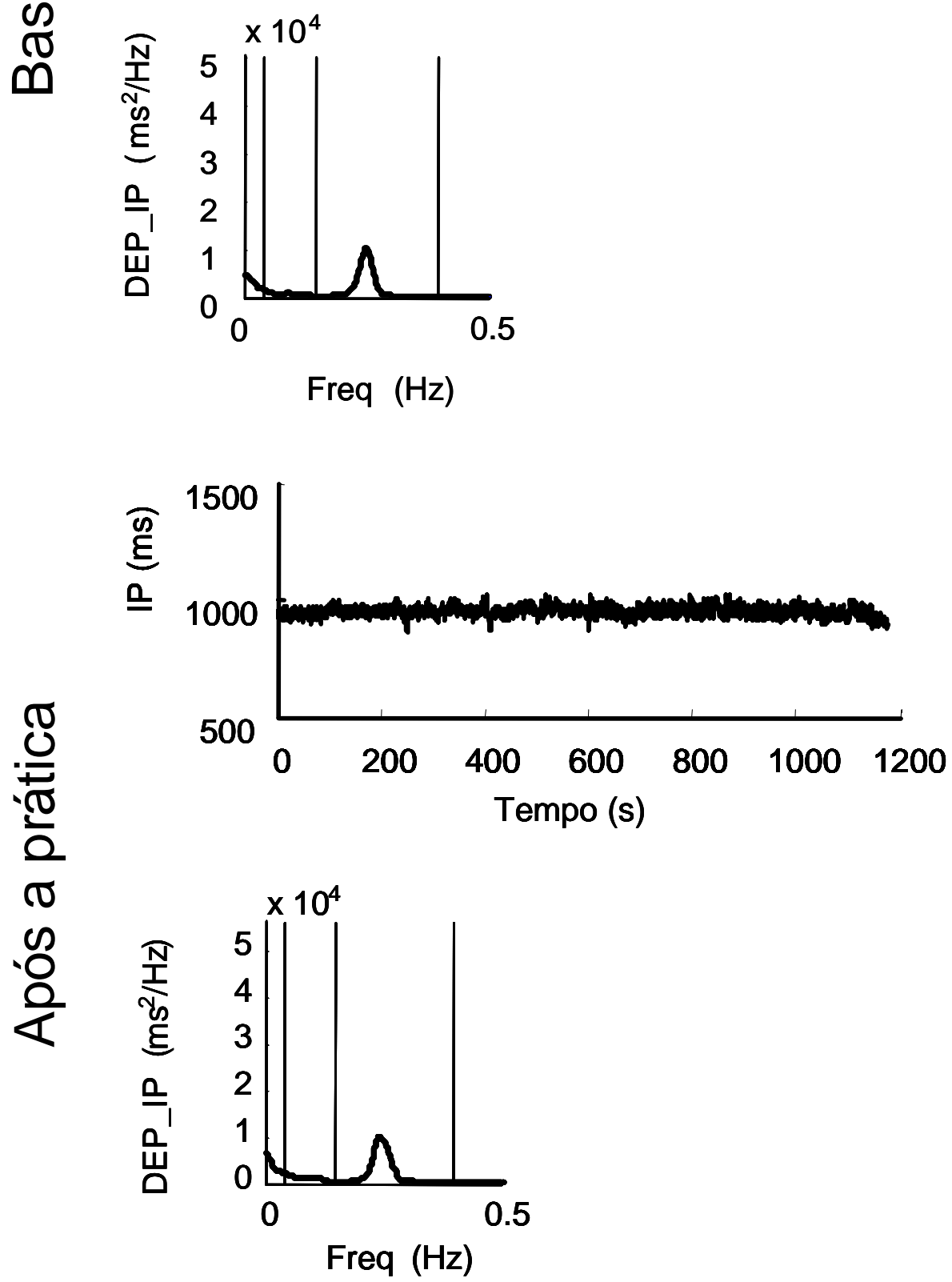
ANEXO IV - Resultados individuais. Séries temporais, DEP e DEP IP (cont.) Controle 4 nas condições BASAL e APÓS A PRÁTICA.
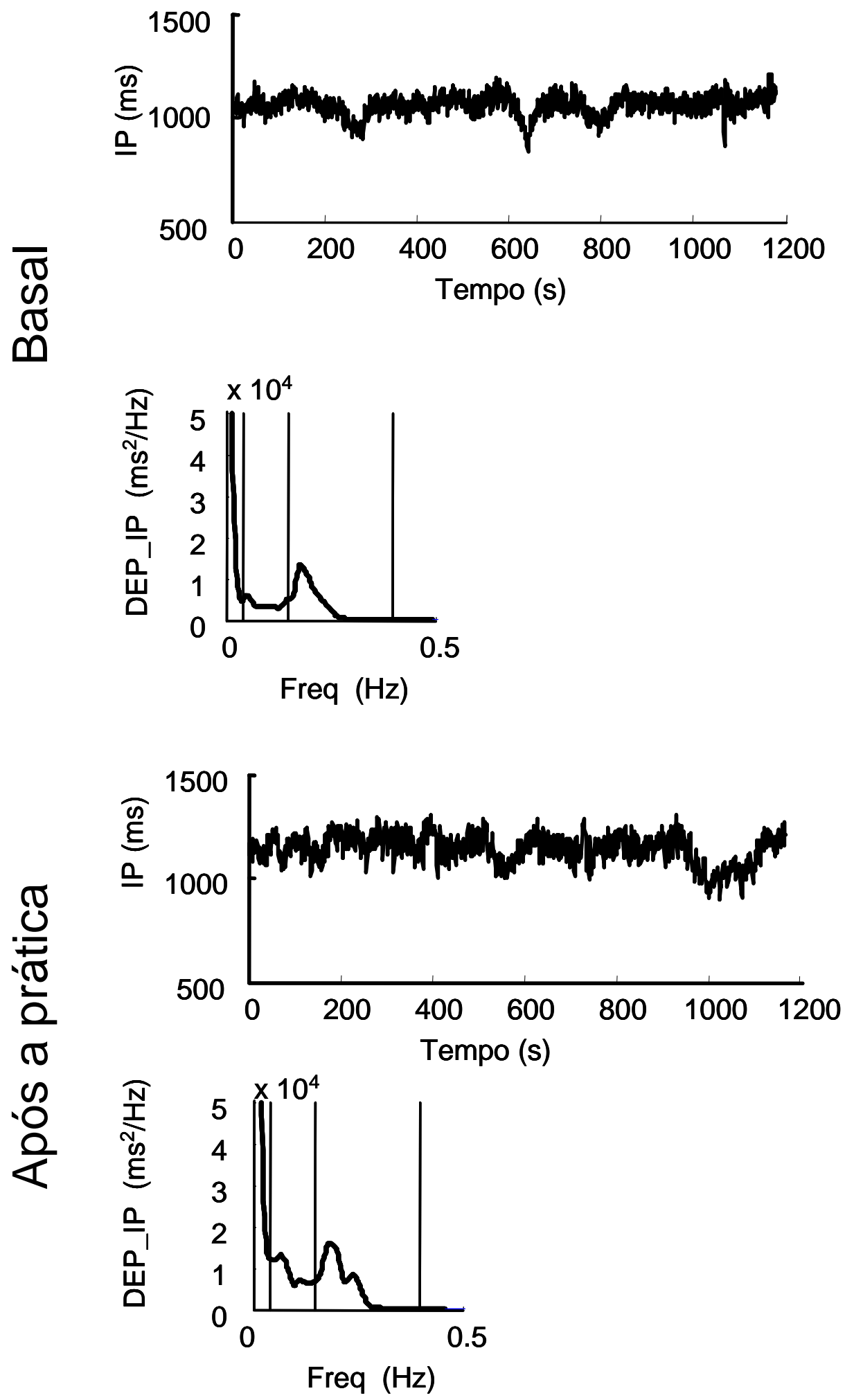
ANEXO IV - Resultados individuais. Séries temporais, DEP e DEP IP (cont.) Controle 5 nas condições BASAL e APÓS A PRÁTICA.
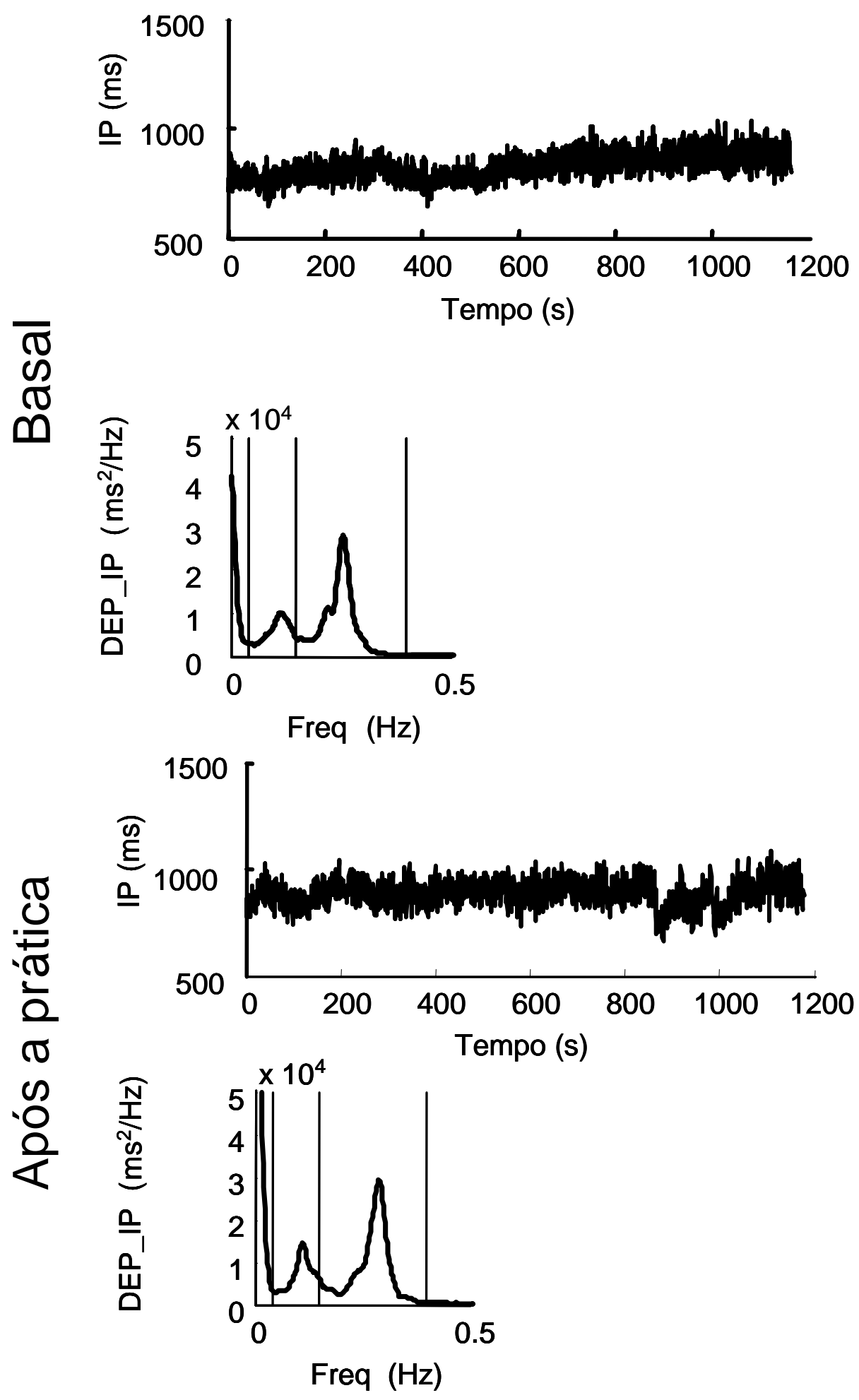
ANEXO IV - Resultados individuais. Séries temporais, DEP e DEP IP (cont.) Controle 6 nas condições BASAL e APÓS A PRÁTICA.

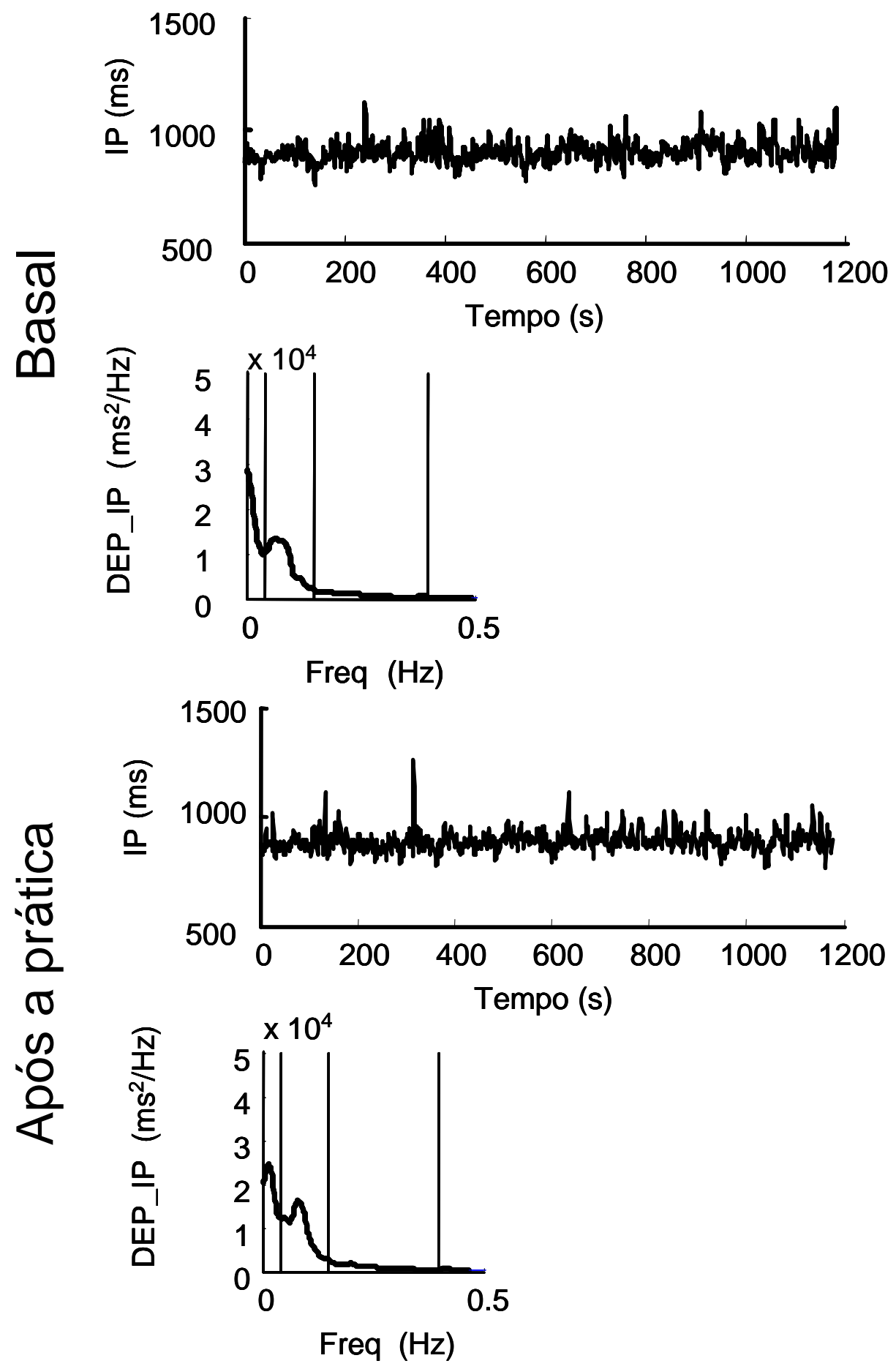


ANEXO IV - Resultados individuais. Séries temporais, DEP e DEP IP (cont.) Controle 7 nas condições BASAL e APÓS A PRÁTICA.

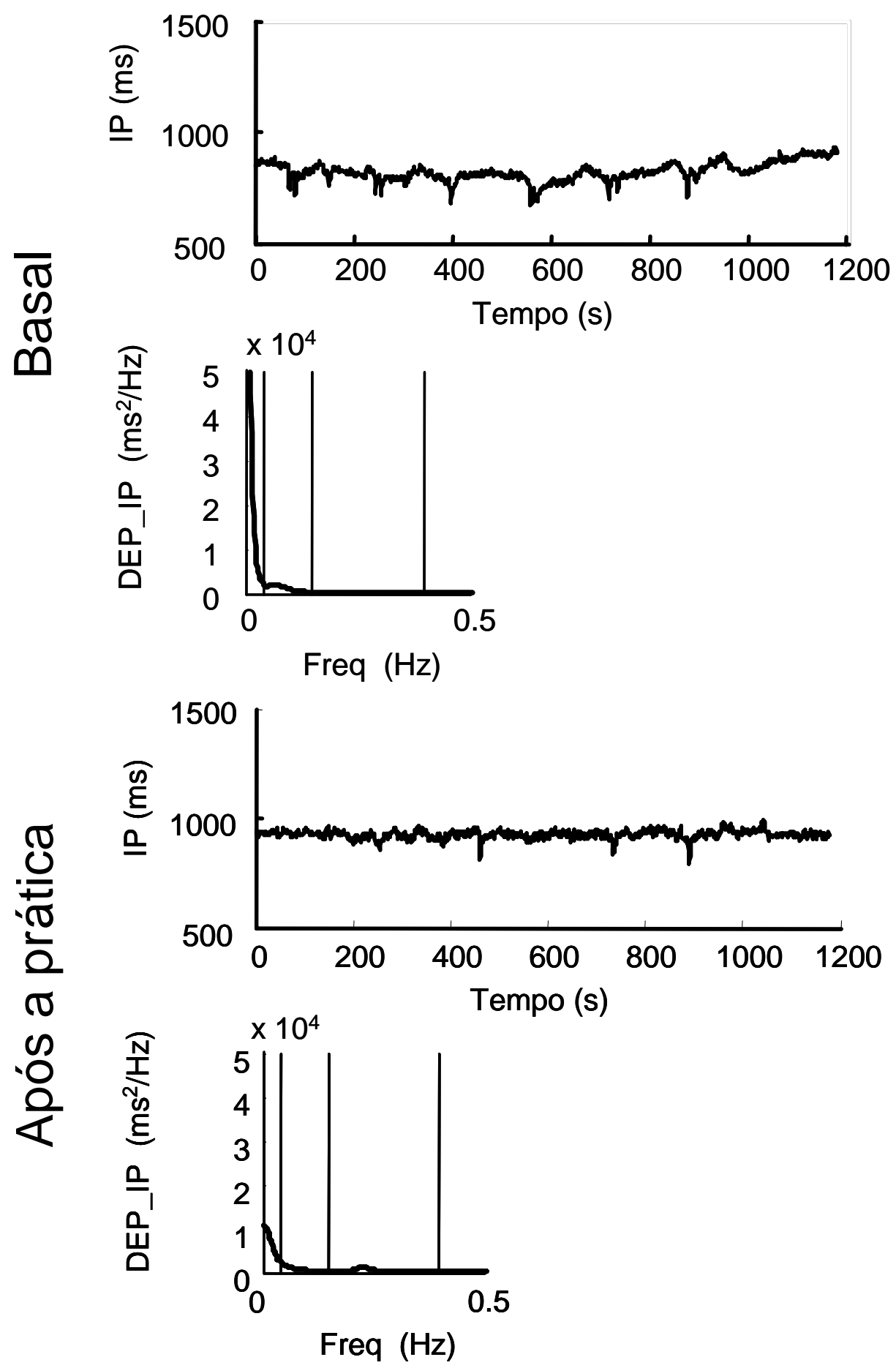


ANEXO IV - Resultados individuais. Séries temporais, DEP e DEP IP (cont.) Controle 8 nas condições BASAL e APÓS A PRÁTICA.

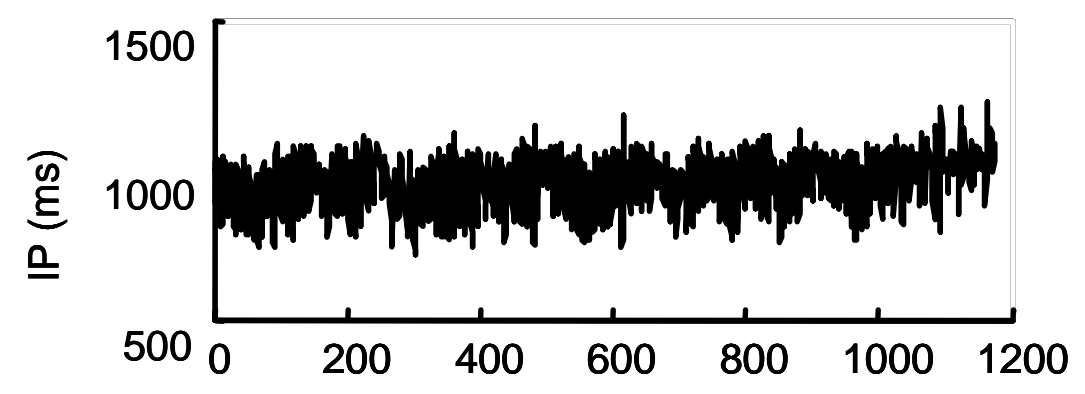

$\bar{\sigma}$
ธิ
ஸ
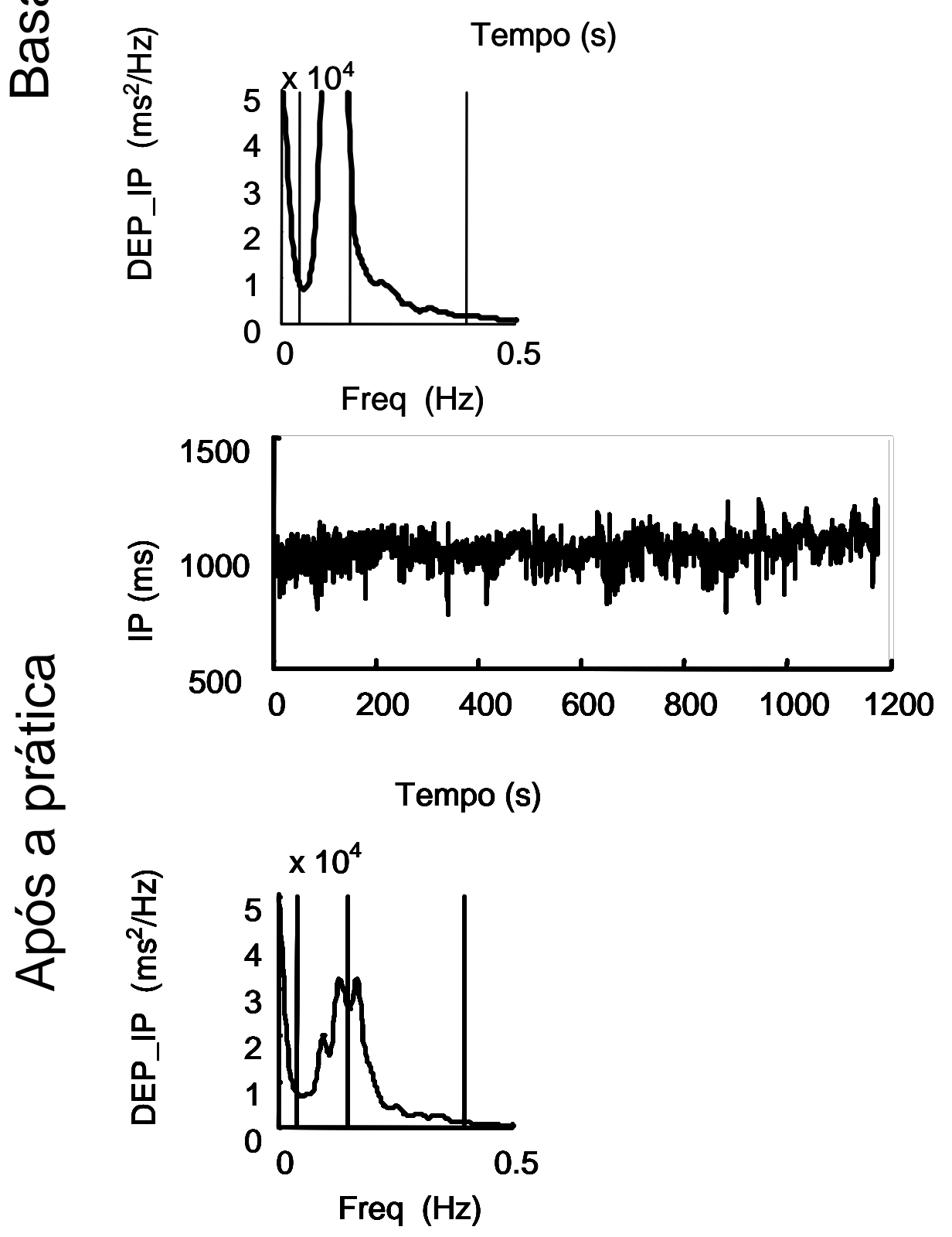


\section{ANEXO V - Resultados da análise estatística entre os grupos IOGA e CT}

Two Way Analysis of Variance Wednesday, February 02, 2005, 08:59:43

Data source: Data 1 in Notebook

Balanced Design

Dependent Variable: FC

Normality Test: Passed $(\mathrm{P}>0,200)$

Equal Variance Test: $\quad$ Passed $(\mathrm{P}=0,929)$

$\begin{array}{llllll}\text { Source of Variation } & \text { DF } & \text { SS } & \text { MS } & \text { F } & \text { P } \\ \text { F1 } & 1 & 231,125 & 231,125 & 6,091 & 0,020 \\ \text { F2 } & 1 & 64,411 & 64,411 & 1,698 & 0,203 \\ \text { F1 x F2 } & 1 & 0,551 & 0,551 & 0,0145 & 0,905 \\ \text { Residual } & 28 & 1062,447 & 37,945 & & \\ \text { Total } & 31 & 1358,535 & 43,824 & & \end{array}$

The difference in the mean values among the different levels of F1 is greater than would be expected by chance after allowing for effects of differences in F2. There is a statistically significant difference $(\mathrm{P}=0,020)$. To isolate which group(s) differ from the others use a multiple comparison procedure.

The difference in the mean values among the different levels of F2 is not great enough to exclude the possibility that the difference is just due to random sampling variability after allowing for the effects of differences in F1. There is not a statistically significant difference $(P=0,203)$.

The effect of different levels of F1 does not depend on what level of F2 is present. There is not a statistically significant interaction between F1 and F2. $(\mathrm{P}=0,905)$

Power of performed test with alpha $=0,0500$ : for $\mathrm{F} 1: 0,583$

Power of performed test with alpha $=0,0500$ : for F2 : 0,119

Power of performed test with alpha $=0,0500$ : for F1 x F2 : 0,0500

Least square means for F1:

Group Mean

IOGA 58,875

CTRL 64,250

Std Err of LS Mean = 1,540

Least square means for F2 :

Group Mean

BAS $\quad 62,981$

APOS 60,144

Std Err of LS Mean = 1,540

Least square means for F1 x F2:

Group Mean

IOGA x BAS $\quad 60,162$

IOGA $x$ APOS 57,587

CTRL x BAS $\quad 65,800$

CTRL $x$ APOS 62,700

Std Err of LS Mean =2,178

All Pairwise Multiple Comparison Procedures (Bonferroni t-test): 
ANEXO V - Resultados da análise estatística entre os grupos IOGA e CT (cont.)

Comparisons for factor: F1

Comparison

Diff of Means

CTRL vs. IOGA

5,375

$\mathrm{P} \quad \mathrm{P}<0,050$

$2,468 \quad 0,020 \quad$ Yes

Comparisons for factor: F2

$\begin{array}{lllll}\text { Comparison } & \text { Diff of Means } & \mathrm{t} & \mathrm{P} & \mathrm{P}<0,050 \\ \text { BAS vs. APOS } & 2,838 & 1,303 & 0,203 & \text { No }\end{array}$

Comparisons for factor: F2 within IOGA

$\begin{array}{lllll}\text { Comparison } & \text { Diff of Means } & \mathrm{t} & \mathrm{P} & \mathrm{P}<0,05 \\ \text { BAS vs. APOS } & 2,575 & 0,836 & 0,410 & \text { No }\end{array}$

Comparisons for factor: F2 within CTRL

$\begin{array}{lllll}\text { Comparison } & \text { Diff of Means } & \mathrm{t} & \mathrm{P} & \mathrm{P}<0,05 \\ \text { BAS vs. APOS } & 3,100 & 1,007 & 0,323 & \text { No }\end{array}$

Comparisons for factor: F1 within BAS

$\begin{array}{lllll}\text { Comparison } & \text { Diff of Means } & \mathrm{t} & \mathrm{P} & \mathrm{P}<0,05 \\ \text { CTRL vs. IOGA } & 5,638 & 1,830 & 0,078 & \text { No }\end{array}$

Comparisons for factor: F1 within APOS

$\begin{array}{lllll}\text { Comparison } & \text { Diff of Means } & \mathrm{t} & \mathrm{P} & \mathrm{P}<0,05 \\ \text { CTRL vs. IOGA } & 5,112 & 1,660 & 0,108 & \text { No }\end{array}$




\section{ANEXO V - Resultados da análise estatística entre os grupos IOGA e CT (cont.)}

Two Way Analysis of Variance Wednesday, February 02, 2005, 09:00:07

Data source: Data 1 in Notebook

Balanced Design

Dependent Variable: DFC

Normality Test: Passed $(\mathrm{P}>0,200)$

Equal Variance Test: $\quad$ Passed $(\mathrm{P}=0,989)$

$\begin{array}{lllllc}\text { Source of Variation } & \text { DF } & \text { SS } & \text { MS } & \text { F } & \text { P } \\ \text { F1 } & 1 & 2,258 & 2,258 & 0,967 & 0,334 \\ \text { F2 } & 1 & 1,015 & 1,015 & 0,435 & 0,515 \\ \text { F1 x F2 } & 1 & 0,525 & 0,525 & 0,225 & 0,639 \\ \text { Residual } & 28 & 65,404 & 2,336 & & \\ \text { Total } & 31 & 69,202 & 2,232 & & \end{array}$

The difference in the mean values among the different levels of F1 is not great enough to exclude the possibility that the difference is just due to random sampling variability after allowing for the effects of differences in F2. There is not a statistically significant difference $(\mathrm{P}=0,334)$.

The difference in the mean values among the different levels of F2 is not great enough to exclude the possibility that the difference is just due to random sampling variability after allowing for the effects of differences in F1. There is not a statistically significant difference $(\mathrm{P}=0,515)$.

The effect of different levels of F1 does not depend on what level of F2 is present. There is not a statistically significant interaction between F1 and F2. $(\mathrm{P}=0,639)$

Power of performed test with alpha $=0,0500$ : for $\mathrm{F} 1: 0,0500$

Power of performed test with alpha $=0,0500$ : for $\mathrm{F} 2: 0,0500$

Power of performed test with alpha $=0,0500$ : for F1 x F2 : 0,0500

Least square means for F1:

Group Mean

IOGA 4,050

CTRL 3,519

Std Err of LS Mean = 0,382

Least square means for F2:

Group Mean

BAS $\quad 3,962$

APOS 3,606

Std Err of LS Mean = 0,382

Least square means for F1 x F2:

Group Mean

IOGA x BAS 4,100

IOGA $x$ APOS 4,000

CTRL x BAS 3,825

CTRL x APOS 3,213

Std Err of LS Mean $=0,540$ 


\section{ANEXO V - Resultados da análise estatística entre os grupos IOGA e CT (cont.)}

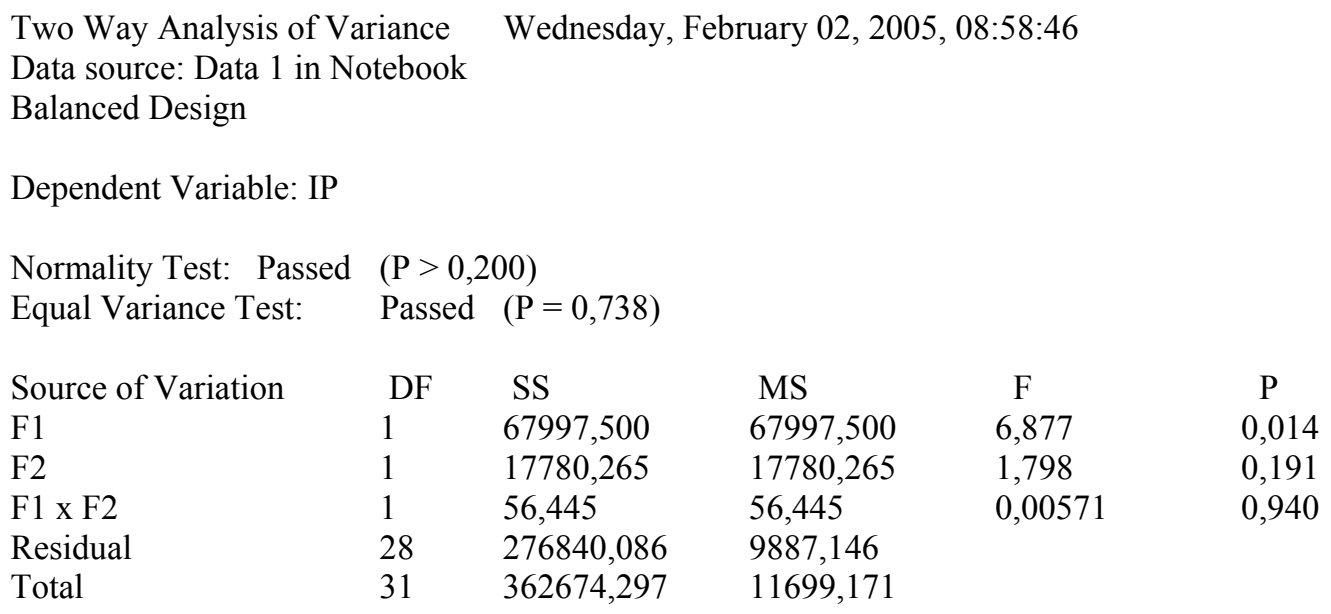

The difference in the mean values among the different levels of F1 is greater than would be expected by chance after allowing for effects of differences in $F 2$. There is a statistically significant difference $(P=0,014)$. To isolate which group(s) differ from the others use a multiple comparison procedure.

The difference in the mean values among the different levels of F2 is not great enough to exclude the possibility that the difference is just due to random sampling variability after allowing for the effects of differences in $\mathrm{F} 1$. There is not a statistically significant difference $(\mathrm{P}=0,191)$.

The effect of different levels of F1 does not depend on what level of F2 is present. There is not a statistically significant interaction between $\mathrm{F} 1$ and $\mathrm{F} 2$. $(\mathrm{P}=0,940)$

Power of performed test with alpha $=0,0500:$ for $\mathrm{F} 1: 0,650$

Power of performed test with alpha $=0,0500$ : for F2 : 0,130

Power of performed test with alpha $=0,0500$ : for F1 x F2 : 0,0500

Least square means for F1:

Group Mean

IOGA 1036,037

CTRL 943,844

Std Err of LS Mean $=24,859$

Least square means for F2:

Group Mean

BAS 966,369

APOS 1013,513

Std Err of LS Mean $=24,859$

Least square means for F1 x F2:

$\begin{array}{ll}\text { Group } & \text { Mean } \\ \text { IOGA x BAS } & 1011,138 \\ \text { IOGA x APOS } & 1060,938 \\ \text { CTRL x BAS } & 921,600 \\ \text { CTRL x APOS } & 966,088\end{array}$

Std Err of LS Mean $=35,155$

All Pairwise Multiple Comparison Procedures (Bonferroni t-test): 
ANEXO V - Resultados da análise estatística entre os grupos IOGA e CT (cont.)

Comparisons for factor: F1

Comparison Diff of Means $\mathrm{t} \quad \mathrm{P} \quad \mathrm{P}<0,050$

IOGA vs. CTRL 92,194 2,622 $0,014 \quad$ Yes

Comparisons for factor: F2

$\begin{array}{lllll}\text { Comparison } & \text { Diff of Means } & \mathrm{t} & \mathrm{P} & \mathrm{P}<0,050 \\ \text { APOS vs. BAS } & 47,144 & 1,341 & 0,191 & \text { No }\end{array}$

Comparisons for factor: F2 within IOGA

Comparison Diff of Means $t \quad P \quad \mathrm{P}<0,05$

APOS vs. BAS 49,800 $\quad 1,002 \quad 0,325$ No

Comparisons for factor: F2 within CTRL

$\begin{array}{lllll}\text { Comparison } & \text { Diff of Means } & \mathrm{t} & \mathrm{P} & \mathrm{P}<0,05\end{array}$

APOS vs. BAS 44,488 $\quad 0,895 \quad 0,379$ No

Comparisons for factor: F1 within BAS

Comparison Diff of Means $t \quad P \quad \mathrm{P}<0,05$

IOGA vs. CTRL 89,538 $1,801 \quad 0,082$ No

Comparisons for factor: F1 within APOS

$\begin{array}{lllll}\text { Comparison } & \text { Diff of Means } & \mathrm{t} & \mathrm{P} & \mathrm{P}<0,05 \\ \text { IOGA vs. CTRL } & 94,850 & 1,908 & 0,067 & \text { No }\end{array}$




\section{ANEXO V - Resultados da análise estatística entre os grupos IOGA e CT (cont.)}

\begin{tabular}{|c|c|c|c|c|c|}
\hline \multicolumn{2}{|c|}{$\begin{array}{l}\text { Two Way Analysis of Variance } \\
\text { Data source: Data } 1 \text { in Notebook } \\
\text { Balanced Design }\end{array}$} & \multicolumn{3}{|c|}{ Wednesday, February 02, 2005, 08:59:21 } & \\
\hline \multicolumn{6}{|l|}{ Dependent Variable: DIP } \\
\hline $\begin{array}{l}\text { Normality Test: Passed } \\
\text { Equal Variance Test: }\end{array}$ & $\begin{array}{l}(\mathrm{P}=0, \\
\text { Passed }\end{array}$ & $\begin{array}{l}\text { 144) } \\
(\mathrm{P}=0,812)\end{array}$ & & & \\
\hline Source of Variation & DF & SS & MS & $\mathrm{F}$ & $\mathrm{P}$ \\
\hline F1 & 1 & 2504,550 & 2504,550 & 4,764 & 0,038 \\
\hline F2 & 1 & 0,475 & 0,475 & 0,000904 & 0,976 \\
\hline $\mathrm{F} 1 \times \mathrm{F} 2$ & 1 & 122,853 & 122,853 & 0,234 & 0,633 \\
\hline Residual & 28 & 14720,339 & 525,726 & & \\
\hline Total & 31 & 17348,217 & 559,620 & & \\
\hline
\end{tabular}

The difference in the mean values among the different levels of F1 is greater than would be expected by chance after allowing for effects of differences in $F 2$. There is a statistically significant difference $(P=0,038)$. To isolate which group(s) differ from the others use a multiple comparison procedure.

The difference in the mean values among the different levels of F2 is not great enough to exclude the possibility that the difference is just due to random sampling variability after allowing for the effects of differences in $\mathrm{F} 1$. There is not a statistically significant difference $(\mathrm{P}=0,976)$.

The effect of different levels of F1 does not depend on what level of F2 is present. There is not a statistically significant interaction between $\mathrm{F} 1$ and $\mathrm{F} 2$. $(\mathrm{P}=0,633)$

Power of performed test with alpha $=0,0500:$ for $\mathrm{F} 1: 0,454$

Power of performed test with alpha $=0,0500$ : for $\mathrm{F} 2: 0,0500$

Power of performed test with alpha $=0,0500$ : for F1 x F2 : 0,0500

Least square means for F1:

Group Mean

IOGA 68,806

CTRL 51,113

Std Err of LS Mean = 5,732

Least square means for F2:

Group Mean

BAS $\quad 59,837$

APOS 60,081

Std Err of LS Mean $=5,732$

Least square means for F1 x F2:

$\begin{array}{ll}\text { Group } & \text { Mean } \\ \text { IOGA x BAS } & 66,725 \\ \text { IOGA x APOS } & 70,887 \\ \text { CTRL x BAS } & 52,950 \\ \text { CTRL x APOS } & 49,275\end{array}$

Std Err of LS Mean = 8,107

All Pairwise Multiple Comparison Procedures (Bonferroni t-test): 
ANEXO V - Resultados da análise estatística entre os grupos IOGA e CT (cont.)

Comparisons for factor: F1

Comparison Diff of Means $\quad t \quad P \quad P<0,050$

IOGA vs. CTRL 17,694 2,183 $0,038 \quad$ Yes

Comparisons for factor: F2

$\begin{array}{lllll}\text { Comparison } & \text { Diff of Means } & \mathrm{t} & \mathrm{P} & \mathrm{P}<0,050 \\ \text { APOS vs. BAS } & 0,244 & 0,0301 & 0,976 & \text { No }\end{array}$

Comparisons for factor: F2 within IOGA

Comparison Diff of Means $t \quad P \quad P<0,05$

APOS vs. BAS 4,162 $\quad 0,363 \quad 0,719$ No

Comparisons for factor: F2 within CTRL

$\begin{array}{lllll}\text { Comparison } & \text { Diff of Means } & t & P & P<0,05\end{array}$

BAS vs. APOS $3,675 \quad 0,321 \quad 0,751 \quad$ No

Comparisons for factor: F1 within BAS

Comparison Diff of Means $t \quad \mathrm{P} \quad \mathrm{P}<0,05$

IOGA vs. CTRL 13,775 $\quad 1,202 \quad 0,240 \quad$ No

Comparisons for factor: F1 within APOS

$\begin{array}{lllll}\text { Comparison } & \text { Diff of Means } & \mathrm{t} & \mathrm{P} & \mathrm{P}<0,05 \\ \text { IOGA vs. CTRL } & 21,612 & 1,885 & 0,070 & \text { No }\end{array}$




\section{ANEXO V - Resultados da análise estatística entre os grupos IOGA e CT (cont.)}

\begin{tabular}{|c|c|c|c|c|c|}
\hline \multicolumn{2}{|c|}{$\begin{array}{l}\text { Two Way Analysis of Variance } \\
\text { Data source: Data } 1 \text { in Notebook } \\
\text { Balanced Design }\end{array}$} & \multicolumn{4}{|c|}{ Wednesday, February 02, 2005, 08:57:53 } \\
\hline \multicolumn{6}{|l|}{ Dependent Variable: FR } \\
\hline Normality Test: Passed & $(\mathrm{P}=0,1$ & 54) & & & \\
\hline Equal Variance Test: & Passed & $(\mathrm{P}=0,602)$ & & & \\
\hline Source of Variation & $\mathrm{DF}$ & SS & MS & $\mathrm{F}$ & $\mathrm{P}$ \\
\hline $\mathrm{F} 1$ & 1 & 3,315 & 3,315 & 0,380 & 0,542 \\
\hline F2 & 1 & 0,878 & 0,878 & 0,101 & 0,753 \\
\hline $\mathrm{F} 1 \times \mathrm{F} 2$ & 1 & 0,690 & 0,690 & 0,0792 & 0,780 \\
\hline Residual & 28 & 244,086 & 8,717 & & \\
\hline Total & 31 & 248,970 & 8,031 & & \\
\hline
\end{tabular}

The difference in the mean values among the different levels of F1 is not great enough to exclude the possibility that the difference is just due to random sampling variability after allowing for the effects of differences in F2. There is not a statistically significant difference $(\mathrm{P}=0,542)$.

The difference in the mean values among the different levels of F2 is not great enough to exclude the possibility that the difference is just due to random sampling variability after allowing for the effects of differences in $\mathrm{F} 1$. There is not a statistically significant difference $(\mathrm{P}=0,753)$.

The effect of different levels of F1 does not depend on what level of F2 is present. There is not a statistically significant interaction between $\mathrm{F} 1$ and $\mathrm{F} 2$. $(\mathrm{P}=0,780)$

Power of performed test with alpha $=0,0500:$ for $\mathrm{F} 1: 0,0500$

Power of performed test with alpha $=0,0500$ : for F2 : 0,0500

Power of performed test with alpha $=0,0500$ : for F1 x F2 : 0,0500

Least square means for F1:

Group Mean

IOGA 14,019

CTRL 13,375

Std Err of LS Mean $=0,738$

Least square means for F2:

Group Mean

BAS 13,531

APOS 13,863

Std Err of LS Mean $=0,738$

Least square means for F1 x F2:

$\begin{array}{ll}\text { Group } & \text { Mean } \\ \text { IOGA x BAS } & 14,000 \\ \text { IOGA x APOS } & 14,038 \\ \text { CTRL x BAS } & 13,062 \\ \text { CTRL x APOS } & 13,688\end{array}$

Std Err of LS Mean = 1,044 


\section{ANEXO V - Resultados da análise estatística entre os grupos IOGA e CT (cont.)}

Two Way Analysis of Variance Wednesday, February 02, 2005, 08:58:25

Data source: Data 1 in Notebook

Balanced Design

Dependent Variable: DFR

Normality Test: Passed $(\mathrm{P}=0,016)$

Equal Variance Test: $\quad$ Passed $(\mathrm{P}=0,443)$

Source of Variation $\quad$ DF $\quad$ SS $\quad$ MS $\quad$ F $\quad$ P

$\begin{array}{llllll}\text { F1 } & 1 & 2,365 & 2,365 & 3,126 & 0,088\end{array}$

$\begin{array}{llllll}\text { F2 } & 1 & 0,525 & 0,525 & 0,694 & 0,412\end{array}$

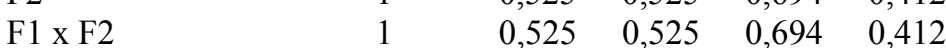

Residual $\quad 28 \quad 21,189 \quad 0,757$

Total $\quad 31 \quad 24,605 \quad 0,794$

The difference in the mean values among the different levels of F1 is not great enough to exclude the possibility that the difference is just due to random sampling variability after allowing for the effects of differences in F2. There is not a statistically significant difference $(\mathrm{P}=0,088)$.

The difference in the mean values among the different levels of F2 is not great enough to exclude the possibility that the difference is just due to random sampling variability after allowing for the effects of differences in F1. There is not a statistically significant difference $(\mathrm{P}=0,412)$.

The effect of different levels of F1 does not depend on what level of F2 is present. There is not a statistically significant interaction between F1 and F2. $(\mathrm{P}=0,412)$

Power of performed test with alpha $=0,0500$ : for $\mathrm{F} 1: 0,276$

Power of performed test with alpha $=0,0500$ : for F2 : 0,0500

Power of performed test with alpha $=0,0500$ : for F1 x F2 : 0,0500

Least square means for F1:

Group Mean

IOGA 2,844

CTRL 2,300

Std Err of LS Mean = 0,217

Least square means for F2 :

Group Mean

BAS 2,444

APOS 2,700

Std Err of LS Mean = 0,217

Least square means for F1 x F2:

$\begin{array}{ll}\text { Group } & \text { Mean } \\ \text { IOGA x BAS } & 2,587 \\ \text { IOGA x APOS } & 3,100 \\ \text { CTRL x BAS } & 2,300 \\ \text { CTRL x APOS } & 2,300 \\ \text { Std Err of LS Mean }=0,308\end{array}$

Std Err of LS Mean $=0,308$ 


\title{
ANEXO V - Resultados da análise estatística entre os grupos IOGA e CT (cont.)
}

\author{
Two Way Analysis of Variance Wednesday, February 02, 2005, 09:01:24 \\ Data source: Data 1 in Notebook \\ Balanced Design \\ Dependent Variable: PTOT \\ Normality Test: Passed $(\mathrm{P}=0,120)$ \\ Equal Variance Test: $\quad$ Passed $(P=0,434)$

$\begin{array}{lllllc}\text { Source of Variation } & \text { DF } & \text { SS } & \text { MS } & \text { F } & \text { P } \\ \text { F1 } & 1 & 36100141,778 & 36100141,778 & 4,339 & 0,046 \\ \text { F2 } & 1 & 641193,190 & 641193,190 & 0,0771 & 0,783 \\ \text { F1 x F2 } & 1 & 739966,538 & 739966,538 & 0,0889 & 0,768 \\ \text { Residual } & 28 & 232956805,241 & 8319885,901 & & \\ \text { Total } & 31 & 270438106,747 & 8723809,895 & & \end{array}$

The difference in the mean values among the different levels of F1 is greater than would be expected by chance after allowing for effects of differences in $F 2$. There is a statistically significant difference $(P=0,046)$. To isolate which group(s) differ from the others use a multiple comparison procedure.

The difference in the mean values among the different levels of F2 is not great enough to exclude the possibility that the difference is just due to random sampling variability after allowing for the effects of differences in $\mathrm{F} 1$. There is not a statistically significant difference $(\mathrm{P}=0,783)$.

The effect of different levels of F1 does not depend on what level of F2 is present. There is not a statistically significant interaction between $\mathrm{F} 1$ and $\mathrm{F} 2$. $(\mathrm{P}=0,768)$

Power of performed test with alpha $=0,0500$ : for $\mathrm{F} 1: 0,410$

Power of performed test with alpha $=0,0500$ : for $\mathrm{F} 2: 0,0500$

Power of performed test with alpha $=0,0500$ : for F1 x F2:0,0500

Least square means for F1:

Group Mean

IOGA 4762,444

CTRL 2638,175

Std Err of LS Mean $=721,105$

Least square means for F2 :

Group Mean

BAS $\quad 3558,756$

APOS 3841,863

Std Err of LS Mean $=721,105$

Least square means for F1 x F2:

$\begin{array}{ll}\text { Group } & \text { Mean } \\ \text { IOGA x BAS } & 4468,825 \\ \text { IOGA x APOS } & 5056,063 \\ \text { CTRL x BAS } & 2648,688 \\ \text { CTRL x APOS } & 2627,663\end{array}$

CTRL x APOS 2627,663
Std Err of LS Mean $=1019,797$

All Pairwise Multiple Comparison Procedures (Bonferroni t-test): 


\section{ANEXO V - Resultados da análise estatística entre os grupos IOGA e CT (cont.)}

Comparisons for factor: F1

Comparison Diff of Means $\quad t \quad P \quad P<0,050$

IOGA vs. CTRL 2124,269 2,083 0,046 Yes

Comparisons for factor: F2

$\begin{array}{lllll}\text { Comparison } & \text { Diff of Means } & \mathrm{t} & \mathrm{P} & \mathrm{P}<0,050 \\ \text { APOS vs. BAS } & 283,106 & 0,278 & 0,783 & \text { No }\end{array}$

Comparisons for factor: F2 within IOGA

Comparison Diff of Means $t \quad P \quad \mathrm{P}<0,05$

APOS vs. BAS 587,238 $0,407 \quad 0,687 \quad$ No

Comparisons for factor: F2 within CTRL
Comparison
Diff of Means
t
$\mathrm{P} \quad \mathrm{P}<0,05$
BAS vs. APOS 21,025
$0,0146 \quad 0,988 \quad$ No

Comparisons for factor: F1 within BAS

$\begin{array}{lllll}\text { Comparison } & \text { Diff of Means } & \mathrm{t} & \mathrm{P} & \mathrm{P}<0,05 \\ \text { IOGA vs. CTRL } & 1820,137 & 1,262 & 0,217 & \text { No }\end{array}$

Comparisons for factor: F1 within APOS

$\begin{array}{lllll}\text { Comparison } & \text { Diff of Means } & \mathrm{t} & \mathrm{P} & \mathrm{P}<0,05 \\ \text { IOGA vs. CTRL } & 2428,400 & 1,684 & 0,103 & \text { No }\end{array}$




\title{
ANEXO V - Resultados da análise estatística entre os grupos IOGA e CT (cont.)
}

\author{
Two Way Analysis of Variance Wednesday, February 02, 2005, 09:01:54 \\ Data source: Data 1 in Notebook \\ Balanced Design \\ Dependent Variable: VAR \\ Normality Test: Passed $(\mathrm{P}>0,200)$ \\ Equal Variance Test: $\quad$ Passed $(P=0,374)$

$\begin{array}{llllcc}\text { Source of Variation } & \text { DF } & \text { SS } & \text { MS } & \text { F } & \text { P } \\ \text { F1 } & 1 & 36226859,200 & 36226859,200 & 4,562 & 0,042 \\ \text { F2 } & 1 & 592388,913 & 592388,913 & 0,0746 & 0,787 \\ \text { F1 x F2 } & 1 & 585605,475 & 585605,475 & 0,0737 & 0,788 \\ \text { Residual } & 28 & 222334296,699 & 7940510,596 & & \\ \text { Total } & 31 & 259739150,287 & 8378682,267 & & \end{array}$

The difference in the mean values among the different levels of F1 is greater than would be expected by chance after allowing for effects of differences in $F 2$. There is a statistically significant difference $(P=0,042)$. To isolate which group(s) differ from the others use a multiple comparison procedure.

The difference in the mean values among the different levels of F2 is not great enough to exclude the possibility that the difference is just due to random sampling variability after allowing for the effects of differences in $\mathrm{F} 1$. There is not a statistically significant difference $(\mathrm{P}=0,787)$.

The effect of different levels of F1 does not depend on what level of F2 is present. There is not a statistically significant interaction between $\mathrm{F} 1$ and $\mathrm{F} 2$. $(\mathrm{P}=0,788)$

Power of performed test with alpha $=0,0500:$ for $\mathrm{F} 1: 0,433$

Power of performed test with alpha $=0,0500$ : for $\mathrm{F} 2: 0,0500$

Power of performed test with alpha $=0,0500$ : for F1 x F2:0,0500

Least square means for F1:

Group Mean

IOGA 4776,388

CTRL 2648,394

Std Err of LS Mean = 704,473

Least square means for F2:

Group Mean

BAS 3576,331

APOS 3848,450

Std Err of LS Mean $=704,473$

Least square means for F1 x F2:

$\begin{array}{ll}\text { Group } & \text { Mean } \\ \text { IOGA x BAS } & 4505,050 \\ \text { IOGA x APOS } & 5047,725 \\ \text { CTRL x BAS } & 2647,613 \\ \text { CTRL x APOS } & 2649,175\end{array}$

Std Err of LS Mean = 996,275

All Pairwise Multiple Comparison Procedures (Bonferroni t-test): 


\section{ANEXO V - Resultados da análise estatística entre os grupos IOGA e CT (cont.)}

Comparisons for factor: F1

Comparison Diff of Means $t \quad P \quad P<0,050$

IOGA vs. CTRL 2127,994 2,136 0,042 Yes

Comparisons for factor: F2

Comparison Diff of Means $\mathrm{t} \quad \mathrm{P} \quad \mathrm{P}<0,050$

$\begin{array}{lllll}\text { APOS vs. BAS } & 272,119 & 0,273 & 0,787 & \text { No }\end{array}$

Comparisons for factor: F2 within IOGA

$\begin{array}{lllll}\text { Comparison } & \text { Diff of Means } & \mathrm{t} & \mathrm{P} & \mathrm{P}<0,05\end{array}$

APOS vs. BAS 542,675 $\quad 0,385 \quad 0,703 \quad$ No

Comparisons for factor: F2 within CTRL

Comparison Diff of Means $t \quad P \quad \mathrm{P}<0,05$

APOS vs. BAS $1,563 \quad 0,001110,999 \quad$ No

Comparisons for factor: F1 within BAS

$\begin{array}{lllll}\text { Comparison } & \text { Diff of Means } & \mathrm{t} & \mathrm{P} & \mathrm{P}<0,05 \\ \text { IOGA vs. CTRL } & 1857,438 & 1,318 & 0,198 & \text { No }\end{array}$

Comparisons for factor: F1 within APOS

Comparison Diff of Means $t \quad \mathrm{P} \quad \mathrm{P}<0,05$

IOGA vs. CTRL 2398,550 $\quad 1,702 \quad 0,100 \quad$ No 


\section{ANEXO V - Resultados da análise estatística entre os grupos IOGA e CT (cont.)}

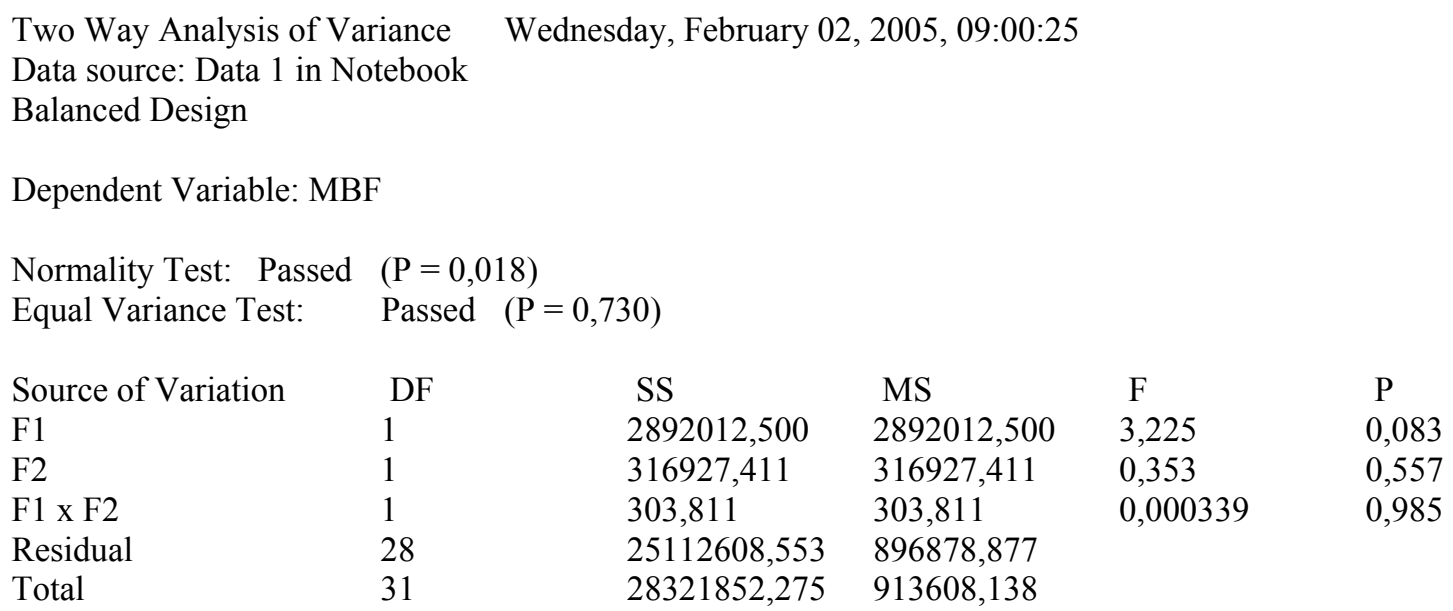

The difference in the mean values among the different levels of F1 is not great enough to exclude the possibility that the difference is just due to random sampling variability after allowing for the effects of differences in F2. There is not a statistically significant difference $(\mathrm{P}=0,083)$.

The difference in the mean values among the different levels of F2 is not great enough to exclude the possibility that the difference is just due to random sampling variability after allowing for the effects of differences in $\mathrm{F} 1$. There is not a statistically significant difference $(\mathrm{P}=0,557)$.

The effect of different levels of F1 does not depend on what level of F2 is present. There is not a statistically significant interaction between $\mathrm{F} 1$ and $\mathrm{F} 2$. $(\mathrm{P}=0,985)$

Power of performed test with alpha $=0,0500:$ for $\mathrm{F} 1: 0,287$

Power of performed test with alpha $=0,0500$ : for $\mathrm{F} 2: 0,0500$

Power of performed test with alpha $=0,0500$ : for F1 x F2 : 0,0500

Least square means for F1:

Group Mean

IOGA 1501,162

CTRL 899,912

Std Err of LS Mean $=236,759$

Least square means for F2:

Group Mean

BAS 1101,019

APOS 1300,056

Std Err of LS Mean = 236,759

Least square means for F1 x F2:

$\begin{array}{ll}\text { Group } & \text { Mean } \\ \text { IOGA x BAS } & 1404,725 \\ \text { IOGA x APOS } & 1597,600 \\ \text { CTRL x BAS } & 797,313 \\ \text { CTRL x APOS } & 1002,512\end{array}$

Std Err of LS Mean $=334,828$ 


\section{ANEXO V - Resultados da análise estatística entre os grupos IOGA e CT (cont.)}

\begin{tabular}{|c|c|c|c|c|c|}
\hline \multicolumn{2}{|c|}{$\begin{array}{l}\text { Two Way Analysis of Variance } \\
\text { Data source: Data } 1 \text { in Notebook } \\
\text { Balanced Design }\end{array}$} & \multicolumn{4}{|c|}{ Wednesday, February $02,2005,09: 00: 50$} \\
\hline \multicolumn{6}{|l|}{ Dependent Variable: BF } \\
\hline $\begin{array}{l}\text { Normality Test: Failed } \\
\text { Equal Variance Test: }\end{array}$ & $\begin{array}{l}(\mathrm{P}=<0 \\
\text { Passed }\end{array}$ & $\begin{array}{l}001) \\
(P=0,805)\end{array}$ & & & \\
\hline Source of Variation & DF & SS & MS & $\mathrm{F}$ & $\mathrm{P}$ \\
\hline F1 & 1 & 7807867,653 & 7807867,653 & 2,352 & 0,136 \\
\hline $\mathrm{F} 2$ & 1 & 164092,883 & 164092,883 & 0,0494 & 0,826 \\
\hline $\mathrm{F} 1 \times \mathrm{F} 2$ & 1 & 435781,140 & 435781,140 & 0,131 & 0,720 \\
\hline Residual & 28 & 92958395,066 & 3319942,681 & & \\
\hline Total & 31 & 101366136,742 & 3269875,379 & & \\
\hline
\end{tabular}

The difference in the mean values among the different levels of F1 is not great enough to exclude the possibility that the difference is just due to random sampling variability after allowing for the effects of differences in F2. There is not a statistically significant difference $(\mathrm{P}=0,136)$.

The difference in the mean values among the different levels of F2 is not great enough to exclude the possibility that the difference is just due to random sampling variability after allowing for the effects of differences in $\mathrm{F} 1$. There is not a statistically significant difference $(\mathrm{P}=0,826)$.

The effect of different levels of F1 does not depend on what level of F2 is present. There is not a statistically significant interaction between $\mathrm{F} 1$ and $\mathrm{F} 2$. $(\mathrm{P}=0,720)$

Power of performed test with alpha $=0,0500$ : for F1 :0,190

Power of performed test with alpha $=0,0500$ : for $\mathrm{F} 2: 0,0500$

Power of performed test with alpha $=0,0500$ : for F1 x F2 : 0,0500

Least square means for F1:

Group Mean

IOGA 1930,175

CTRL 942,256

Std Err of LS Mean $=455,518$

Least square means for F2:

Group Mean

BAS $\quad 1507,825$

APOS 1364,606

Std Err of LS Mean $=455,518$

Least square means for F1 x F2 :

$\begin{array}{ll}\text { Group } & \text { Mean } \\ \text { IOGA x BAS } & 1885,087 \\ \text { IOGA x APOS } & 1975,263 \\ \text { CTRL x BAS } & 1130,563 \\ \text { CTRL x APOS } & 753,950\end{array}$

Std Err of LS Mean = 644,199 


\section{ANEXO V - Resultados da análise estatística entre os grupos IOGA e CT (cont.)}

\begin{tabular}{|c|c|c|c|c|c|}
\hline \multicolumn{2}{|c|}{$\begin{array}{l}\text { Two Way Analysis of Variance } \\
\text { Data source: Data } 1 \text { in Notebook } \\
\text { Balanced Design }\end{array}$} & \multicolumn{4}{|c|}{ Wednesday, February 02, 2005, 09:01:07 } \\
\hline \multicolumn{6}{|l|}{ Dependent Variable: AF } \\
\hline $\begin{array}{l}\text { Normality Test: Passed } \\
\text { Equal Variance Test: }\end{array}$ & $\begin{array}{l}(\mathrm{P}>0,2 \\
\text { Passed }\end{array}$ & $\begin{array}{l}00) \\
(P=0,424)\end{array}$ & & & \\
\hline Source of Variation & DF & SS & MS & $\mathrm{F}$ & $\mathrm{P}$ \\
\hline $\mathrm{F} 1$ & 1 & 2285842,711 & 2285842,711 & 2,897 & 0,100 \\
\hline F2 & 1 & 455582,851 & 455582,851 & 0,577 & 0,454 \\
\hline $\mathrm{F} 1 \times \mathrm{F} 2$ & 1 & 60378,125 & 60378,125 & 0,0765 & 0,784 \\
\hline Residual & 28 & 22091093,193 & 788967,614 & & \\
\hline Total & 31 & 24892896,880 & 802996,674 & & \\
\hline
\end{tabular}

The difference in the mean values among the different levels of F1 is not great enough to exclude the possibility that the difference is just due to random sampling variability after allowing for the effects of differences in F2. There is not a statistically significant difference $(\mathrm{P}=0,100)$.

The difference in the mean values among the different levels of F2 is not great enough to exclude the possibility that the difference is just due to random sampling variability after allowing for the effects of differences in $\mathrm{F} 1$. There is not a statistically significant difference $(\mathrm{P}=0,454)$.

The effect of different levels of F1 does not depend on what level of F2 is present. There is not a statistically significant interaction between $\mathrm{F} 1$ and $\mathrm{F} 2$. $(\mathrm{P}=0,784)$

Power of performed test with alpha $=0,0500$ : for $\mathrm{F} 1: 0,250$

Power of performed test with alpha $=0,0500$ : for $\mathrm{F} 2: 0,0500$

Power of performed test with alpha $=0,0500$ : for F1 x F2 : 0,0500

Least square means for F1:

Group Mean

IOGA 1315,994

CTRL 781,456

Std Err of LS Mean $=222,060$

Least square means for F2:

Group Mean

BAS 929,406

APOS 1168,044

Std Err of LS Mean $=222,060$

Least square means for F1 x F2:

$\begin{array}{ll}\text { Group } & \text { Mean } \\ \text { IOGA x BAS } & 1153,238 \\ \text { IOGA x APOS } & 1478,750 \\ \text { CTRL x BAS } & 705,575 \\ \text { CTRL x APOS } & 857,338\end{array}$

Std Err of LS Mean $=314,040$ 


\section{ANEXO V - Resultados da análise estatística entre os grupos IOGA e CT (cont.)}

\begin{tabular}{|c|c|c|c|c|c|}
\hline \multicolumn{2}{|c|}{$\begin{array}{l}\text { Two Way Analysis of Variance } \\
\text { Data source: Data } 1 \text { in Notebook } \\
\text { Balanced Design }\end{array}$} & \multicolumn{4}{|c|}{ Wednesday, February 02, 2005, 09:02:13 } \\
\hline \multicolumn{6}{|l|}{ Dependent Variable: BFn } \\
\hline $\begin{array}{l}\text { Normality Test: Passed } \\
\text { Equal Variance Test: }\end{array}$ & $\begin{array}{l}(\mathrm{P}>0, \\
\text { Passed }\end{array}$ & $\begin{array}{l}00) \\
(\mathrm{P}=0.790)\end{array}$ & & & \\
\hline Source of Variation & DF & SS & MS & $\mathrm{F}$ & $\mathrm{P}$ \\
\hline F1 & 1 & 599,445 & 599,445 & 1,657 & 0,209 \\
\hline $\mathrm{F} 2$ & 1 & 1,403 & 1,403 & 0,00388 & 0,951 \\
\hline F1 x F2 & 1 & 0,0528 & 0,0528 & 0,000146 & 0,990 \\
\hline Residual & 28 & 10128,489 & 361,732 & & \\
\hline Total & 31 & 10729,390 & 346,109 & & \\
\hline
\end{tabular}

The difference in the mean values among the different levels of F1 is not great enough to exclude the possibility that the difference is just due to random sampling variability after allowing for the effects of differences in F2. There is not a statistically significant difference $(\mathrm{P}=0,209)$.

The difference in the mean values among the different levels of F2 is not great enough to exclude the possibility that the difference is just due to random sampling variability after allowing for the effects of differences in F1. There is not a statistically significant difference $(\mathrm{P}=0,951)$.

The effect of different levels of F1 does not depend on what level of F2 is present. There is not a statistically significant interaction between F1 and F2. $(\mathrm{P}=0,990)$

Power of performed test with alpha $=0,0500$ : for $\mathrm{F} 1: 0,115$

Power of performed test with alpha $=0,0500$ : for F2 : 0,0500

Power of performed test with alpha $=0,0500$ : for F1 x F2 : 0,0500

Least square means for F1:

Group Mean

IOGA 57,225

CTRL 48,569

Std Err of LS Mean $=4,755$

Least square means for $\mathrm{F} 2$ :

Group Mean

BAS $\quad 52,688$

APOS 53,106

Std Err of LS Mean = 4,755

Least square means for F1 x F2:

$\begin{array}{ll}\text { Group } & \text { Mean } \\ \text { IOGA x BAS } & 56,975 \\ \text { IOGA x APOS } & 57,475 \\ \text { CTRL x BAS } & 48,400 \\ \text { CTRL x APOS } & 48,737\end{array}$

Std Err of LS Mean =6,724 


\section{ANEXO V - Resultados da análise estatística entre os grupos IOGA e CT (cont.)}

Two Way Analysis of Variance Wednesday, February 02, 2005, 09:02:33

Data source: Data 1 in Notebook

Balanced Design

Dependent Variable: AFn

Normality Test: Passed $(\mathrm{P}>0,200)$

Equal Variance Test: $\quad$ Passed $(\mathrm{P}=0,147)$

$\begin{array}{llllll}\text { Source of Variation } & \text { DF } & \text { SS } & \text { MS } & \text { F } & \text { P } \\ \text { F1 } & 1 & 359,790 & 359,790 & 0,935 & 0,342 \\ \text { F2 } & 1 & 341,258 & 341,258 & 0,887 & 0,354 \\ \text { F1 x F2 } & 1 & 0,878 & 0,878 & 0,00228 & 0,962 \\ \text { Residual } & 28 & 10773,896 & 384,782 & & \\ \text { Total } & 31 & 11475,822 & 370,188 & & \end{array}$

The difference in the mean values among the different levels of F1 is not great enough to exclude the possibility that the difference is just due to random sampling variability after allowing for the effects of differences in F2. There is not a statistically significant difference $(\mathrm{P}=0,342)$.

The difference in the mean values among the different levels of F2 is not great enough to exclude the possibility that the difference is just due to random sampling variability after allowing for the effects of differences in F1. There is not a statistically significant difference $(\mathrm{P}=0,354)$.

The effect of different levels of F1 does not depend on what level of F2 is present. There is not a statistically significant interaction between F1 and F2. $(\mathrm{P}=0,962)$

Power of performed test with alpha $=0,0500$ : for $\mathrm{F} 1: 0,0500$

Power of performed test with alpha $=0,0500$ : for F2 : 0,0500

Power of performed test with alpha $=0,0500$ : for F1 x F2 : 0,0500

Least square means for F1:

Group Mean

IOGA 41,563

CTRL 48,269

Std Err of LS Mean = 4,904

Least square means for F2:

Group Mean

BAS 41,650

APOS 48,181

Std Err of LS Mean = 4,904

Least square means for F1 x F2:

$\begin{array}{ll}\text { Group } & \text { Mean } \\ \text { IOGA x BAS } & 38,462 \\ \text { IOGA x APOS } & 44,663 \\ \text { CTRL x BAS } & 44,837 \\ \text { CTRL x APOS } & 51,700\end{array}$

Std Err of LS Mean = 6,935 


\section{ANEXO V - Resultados da análise estatística entre os grupos IOGA e CT (cont.)}

\begin{tabular}{|c|c|c|c|c|c|}
\hline \multicolumn{2}{|c|}{$\begin{array}{l}\text { Two Way Analysis of Variance } \\
\text { Data source: Data } 1 \text { in Notebook } \\
\text { Balanced Design }\end{array}$} & \multicolumn{4}{|c|}{ Thursday, June 09, 2005, 11:54: } \\
\hline \multicolumn{6}{|c|}{ Dependent Variable: BF/AF } \\
\hline Normality Test: Failed & $(\mathrm{P}=0.0$ & 07) & & & \\
\hline Equal Variance Test: & Passed & $(\mathrm{P}=0$ & 73) & & \\
\hline Source of Variation & $\mathrm{DF}$ & SS & MS & $\mathrm{F}$ & $\mathrm{P}$ \\
\hline $\mathrm{F} 1$ & 1 & 0.211 & 0.211 & 0.111 & 0.742 \\
\hline F2 & 1 & 1.901 & 1.901 & 0.999 & 0.326 \\
\hline $\mathrm{F} 1 \times \mathrm{F} 2$ & 1 & 0.0450 & 0.0450 & 0.0236 & 0.879 \\
\hline Residual & 28 & 53.297 & 1.903 & & \\
\hline Total & 31 & 55.455 & 1.789 & & \\
\hline
\end{tabular}

The difference in the mean values among the different levels of F1 is not great enough to exclude the possibility that the difference is just due to random sampling variability after allowing for the effects of differences in F2. There is not a statistically significant difference $(\mathrm{P}=0.742)$.

The difference in the mean values among the different levels of F2 is not great enough to exclude the possibility that the difference is just due to random sampling variability after allowing for the effects of differences in F1. There is not a statistically significant difference $(\mathrm{P}=0.326)$.

The effect of different levels of F1 does not depend on what level of F2 is present. There is not a statistically significant interaction between F1 and F2. $(P=0.879)$

Power of performed test with alpha $=0.0500:$ for $\mathrm{F} 1: 0.0500$

Power of performed test with alpha $=0.0500:$ for F $2: 0.0500$

Power of performed test with alpha $=0.0500:$ for F1 x F2 : 0.0500

Least square means for F1:

Group Mean

IOGA 1.769

CTRL 1.606

Std Err of LS Mean $=0.345$

Least square means for F2:

Group Mean

BAS 1.931

APOS 1.444

Std Err of LS Mean $=0.345$

Least square means for F1 x F2:

$\begin{array}{ll}\text { Group } & \text { Mean } \\ \text { IOGA x BAS } & 1.975 \\ \text { IOGA x APOS } & 1.563 \\ \text { CTRL x BAS } & 1.888 \\ \text { CTRL x APOS } & 1.325\end{array}$

Std Err of LS Mean $=0.488$ 


\section{ANEXO VI - Resultados da análise estatística entre os grupos IOGAativ e CTativ.}

Two Way Analysis of Variance Wednesday, February 02, 2005, 09:40:55

Data source: Data 1 in Notebook

Balanced Design

Dependent Variable: FC

Normality Test: Passed $(\mathrm{P}>0.200)$

Equal Variance Test: $\quad$ Passed $(\mathrm{P}=0.799)$

$\begin{array}{llllll}\text { Source of Variation } & \text { DF } & \text { SS } & \text { MS } & \text { F } & \text { P } \\ \text { Col 1 } & 1 & 272.250 & 272.250 & 11.772 & 0.005 \\ \text { Col 2 } & 1 & 19.803 & 19.803 & 0.856 & 0.373 \\ \text { Col 1 x Col 2 } & 1 & 2.250 & 2.250 & 0.0973 & 0.760 \\ \text { Residual } & 12 & 277.515 & 23.126 & & \\ \text { Total } & 15 & 571.818 & 38.121 & & \end{array}$

The difference in the mean values among the different levels of Col 1 is greater than would be expected by chance after allowing for effects of differences in Col 2. There is a statistically significant difference $(\mathrm{P}=0.005)$. To isolate which group(s) differ from the others use a multiple comparison procedure.

The difference in the mean values among the different levels of Col 2 is not great enough to exclude the possibility that the difference is just due to random sampling variability after allowing for the effects of differences in Col 1. There is not a statistically significant difference $(\mathrm{P}=0.373)$.

The effect of different levels of Col 1 does not depend on what level of Col 2 is present. There is not a statistically significant interaction between $\mathrm{Col} 1$ and $\mathrm{Col} 2 .(\mathrm{P}=0.760)$

Power of performed test with alpha $=0.0500$ : for Col $1: 0.866$

Power of performed test with alpha $=0.0500$ : for Col $2: 0.0500$

Power of performed test with alpha $=0.0500$ : for Col 1 x Col $2: 0.0500$

Least square means for Col 1:

Group Mean

IOGA 54.987

CTRL 63.238

Std Err of LS Mean $=1.700$

Least square means for $\mathrm{Col} 2$ :

Group Mean

BAS $\quad 60.225$

APOS 58.000

Std Err of LS Mean $=1.700$

Least square means for Col 1 x Col 2:

$\begin{array}{ll}\text { Group } & \text { Mean } \\ \text { IOGA x BAS } & 56.475 \\ \text { IOGA x APOS } & 53.500 \\ \text { CTRL x BAS } & 63.975 \\ \text { CTRL x APOS } & 62.500\end{array}$

Std Err of LS Mean = 2.404 


\section{ANEXO VI - Resultados da análise estatística entre os grupos IOGAativ e $\underline{\text { CTativ (cont.) }}$}

All Pairwise Multiple Comparison Procedures (Bonferroni t-test):

Comparisons for factor: Col 1

$\begin{array}{lrlll}\text { Comparison } & \text { Diff of Means } & \mathrm{t} & \mathrm{P} & \mathrm{P}<0.050\end{array}$

$\begin{array}{lllll}\text { CTRL vs. IOGA } & 8.250 & 3.431 & 0.005 & \text { Yes }\end{array}$

Comparisons for factor: $\mathrm{Col} 2$

Comparison Diff of Means $\quad \mathrm{t} \quad \mathrm{P} \quad \mathrm{P}<0.050$

$\begin{array}{lllll}\text { BAS vs. APOS } & 2.225 & 0.925 & 0.373 & \text { No }\end{array}$

Comparisons for factor: $\mathrm{Col} 2$ within IOGA

$\begin{array}{lrlll}\text { Comparison } & \text { Diff of Means } & \mathrm{t} & \mathrm{P} & \mathrm{P}<0.05 \\ \text { BAS vs. APOS } & 2.975 & 0.875 & 0.399 & \text { No }\end{array}$

Comparisons for factor: Col 2 within CTRL

$\begin{array}{lllll}\text { Comparison } & \text { Diff of Means } & t & \mathrm{P} & \mathrm{P}<0.05\end{array}$

$\begin{array}{lllll}\text { BAS vs. APOS } & 1.475 & 0.434 & 0.672 & \text { No }\end{array}$

Comparisons for factor: Col 1 within BAS

$\begin{array}{lrlll}\text { Comparison } & \text { Diff of Means } & \mathrm{t} & \mathrm{P} & \mathrm{P}<0.05 \\ \text { CTRL vs. IOGA } & 7.500 & 2.206 & 0.048 & \text { Yes }\end{array}$

Comparisons for factor: Col 1 within APOS

$\begin{array}{lrlll}\text { Comparison } & \text { Diff of Means } & \mathrm{t} & \mathrm{P} & \mathrm{P}<0.05 \\ \text { CTRL vs. IOGA } & 9.000 & 2.647 & 0.021 & \text { Yes }\end{array}$




\section{ANEXO VI - Resultados da análise estatística entre os grupos IOGAativ e CTativ (cont.)}

\begin{tabular}{|c|c|c|c|c|c|}
\hline \multicolumn{2}{|c|}{$\begin{array}{l}\text { Two Way Analysis of Variance } \\
\text { Data source: Data } 1 \text { in Notebook } \\
\text { Balanced Design }\end{array}$} & \multicolumn{4}{|c|}{ Wednesday, February 02, 2005, 09:41:22 } \\
\hline \multicolumn{6}{|l|}{ Dependent Variable: DFC } \\
\hline Normality Test: Passed & $(\mathrm{P}>0.2$ & 00) & & & \\
\hline Equal Variance Test: & Passed & $(\mathrm{P}=0.233)$ & & & \\
\hline Source of Variation & $\mathrm{DF}$ & SS & MS & $\mathrm{F}$ & $\mathrm{P}$ \\
\hline Col 1 & 1 & 9.000 & 9.000 & 7.997 & 0.015 \\
\hline Col 2 & 1 & 0.0900 & 0.0900 & 0.0800 & 0.782 \\
\hline Col 1 x Col 2 & 1 & 0.00250 & 0.00250 & 0.00222 & 0.963 \\
\hline Residual & 12 & 13.505 & 1.125 & & \\
\hline Total & 15 & 22.598 & 1.507 & & \\
\hline
\end{tabular}

The difference in the mean values among the different levels of Col 1 is greater than would be expected by chance after allowing for effects of differences in Col 2. There is a statistically significant difference $(\mathrm{P}=0.015)$. To isolate which group(s) differ from the others use a multiple comparison procedure.

The difference in the mean values among the different levels of Col 2 is not great enough to exclude the possibility that the difference is just due to random sampling variability after allowing for the effects of differences in Col 1. There is not a statistically significant difference $(\mathrm{P}=0.782)$.

The effect of different levels of Col 1 does not depend on what level of Col 2 is present. There is not a statistically significant interaction between $\mathrm{Col} 1$ and $\mathrm{Col} 2 .(\mathrm{P}=0.963)$

Power of performed test with alpha $=0.0500$ : for Col $1: 0.685$

Power of performed test with alpha $=0.0500$ : for Col $2: 0.0500$

Power of performed test with alpha $=0.0500$ : for $\operatorname{Col} 1 \times$ Col $2: 0.0500$

Least square means for Col 1:

Group Mean

IOGA 4.162

CTRL 2.662

Std Err of LS Mean $=0.375$

Least square means for $\mathrm{Col} 2$ :

Group Mean

BAS $\quad 3.337$

APOS 3.487

Std Err of LS Mean $=0.375$

Least square means for Col $1 \times$ Col 2:

$\begin{array}{ll}\text { Group } & \text { Mean } \\ \text { IOGA x BAS } & 4.075 \\ \text { IOGA x APOS } & 4.250 \\ \text { CTRL x BAS } & 2.600 \\ \text { CTRL x APOS } & 2.725\end{array}$

Std Err of LS Mean $=0.530$ 


\section{ANEXO VI - Resultados da análise estatística entre os grupos IOGAativ e $\underline{\text { CTativ (cont.) }}$}

All Pairwise Multiple Comparison Procedures (Bonferroni t-test):

Comparisons for factor: Col 1

$\begin{array}{lrlll}\text { Comparison } & \text { Diff of Means } & \mathrm{t} & \mathrm{P} & \mathrm{P}<0.050\end{array}$

$\begin{array}{lllll}\text { IOGA vs. CTRL } & 1.500 & 2.828 & 0.015 & \text { Yes }\end{array}$

Comparisons for factor: $\mathrm{Col} 2$

Comparison Diff of Means $\mathrm{t} \quad \mathrm{P} \quad \mathrm{P}<0.050$

$\begin{array}{lllll}\text { APOS vs. BAS } & 0.150 & 0.283 & 0.782 & \text { No }\end{array}$

Comparisons for factor: $\mathrm{Col} 2$ within IOGA

$\begin{array}{lrlll}\text { Comparison } & \text { Diff of Means } & \mathrm{t} & \mathrm{P} & \mathrm{P}<0.05 \\ \text { APOS vs. BAS } & 0.175 & 0.233 & 0.819 & \text { No }\end{array}$

Comparisons for factor: Col 2 within CTRL

$\begin{array}{lllll}\text { Comparison } & \text { Diff of Means } & \mathrm{t} & \mathrm{P} & \mathrm{P}<0.05\end{array}$

$\begin{array}{lllll}\text { APOS vs. BAS } & 0.125 & 0.167 & 0.870 & \text { No }\end{array}$

Comparisons for factor: Col 1 within BAS

$\begin{array}{lrlll}\text { Comparison } & \text { Diff of Means } & \mathrm{t} & \mathrm{P} & \mathrm{P}<0.05 \\ \text { IOGA vs. CTRL } & 1.475 & 1.966 & 0.073 & \text { No }\end{array}$

$\begin{array}{lllll}\text { IOGA vs. CTRL } & 1.475 & 1.966 & 0.073 & \text { No }\end{array}$

Comparisons for factor: Col 1 within APOS

$\begin{array}{lllll}\text { Comparison Diff of Means } & t & P & \mathrm{P}<0.05\end{array}$ $\begin{array}{lllll}\text { IOGA vs. CTRL } & 1.525 & 2.033 & 0.065 & \text { No }\end{array}$ 


\section{ANEXO VI - Resultados da análise estatística entre os grupos IOGAativ e CTativ (cont.)}

\begin{tabular}{|c|c|c|c|c|c|}
\hline \multicolumn{2}{|c|}{$\begin{array}{l}\text { Two Way Analysis of Variance } \\
\text { Data source: Data } 1 \text { in Notebook } \\
\text { Balanced Design }\end{array}$} & \multicolumn{4}{|c|}{ Wednesday, February 02, 2005, 09:39:49 } \\
\hline \multicolumn{6}{|l|}{ Dependent Variable: IP } \\
\hline Normality Test: Passed & $(\mathrm{P}=0.0$ & 68) & & & \\
\hline Equal Variance Test: & Passed & $(\mathrm{P}=0.864)$ & & & \\
\hline Source of Variation & $\mathrm{DF}$ & SS & MS & $\mathrm{F}$ & $\mathrm{P}$ \\
\hline Col 1 & 1 & 82742.522 & 82742.522 & 11.345 & 0.006 \\
\hline Col 2 & 1 & 8190.250 & 8190.250 & 1.123 & 0.310 \\
\hline $\mathrm{Col} 1 \times \mathrm{Col} 2$ & 1 & 1207.563 & 1207.563 & 0.166 & 0.691 \\
\hline Residual & 12 & 87517.655 & 7293.138 & & \\
\hline Total & 15 & 179657.990 & 11977.199 & & \\
\hline
\end{tabular}

The difference in the mean values among the different levels of Col 1 is greater than would be expected by chance after allowing for effects of differences in $\mathrm{Col} 2$. There is a statistically significant difference $(\mathrm{P}=0.006)$. To isolate which group(s) differ from the others use a multiple comparison procedure.

The difference in the mean values among the different levels of Col 2 is not great enough to exclude the possibility that the difference is just due to random sampling variability after allowing for the effects of differences in Col 1. There is not a statistically significant difference $(\mathrm{P}=0.310)$.

The effect of different levels of Col 1 does not depend on what level of Col 2 is present. There is not a statistically significant interaction between $\mathrm{Col} 1$ and $\mathrm{Col} 2$. $(\mathrm{P}=0.691)$

Power of performed test with alpha $=0.0500$ : for Col $1: 0.852$

Power of performed test with alpha $=0.0500$ : for Col $2: 0.0596$

Power of performed test with alpha $=0.0500$ : for Col $1 \times$ Col $2: 0.0500$

Least square means for Col 1:

Group Mean

IOGA 1101.588

CTRL 957.763

Std Err of LS Mean $=30.193$

Least square means for Col 2:

Group Mean

BAS $\quad 1007.050$

APOS 1052.300

Std Err of LS Mean $=30.193$

Least square means for Col $1 \times$ Col 2:

$\begin{array}{ll}\text { Group } & \text { Mean } \\ \text { IOGA x BAS } & 1070.275 \\ \text { IOGA x APOS } & 1132.900 \\ \text { CTRL x BAS } & 943.825 \\ \text { CTRL x APOS } & 971.700 \\ \text { Std Err of LS Mean }=42.700\end{array}$




\section{ANEXO VI - Resultados da análise estatística entre os grupos IOGAativ e CTativ (cont.)}

All Pairwise Multiple Comparison Procedures (Bonferroni t-test):

Comparisons for factor: Col 1

$\begin{array}{lrlll}\text { Comparison } & \text { Diff of Means } & \mathrm{t} & \mathrm{P} & \mathrm{P}<0.050 \\ \text { IOGA vs. CTRL } & 143.825 & 3.368 & 0.006 & \mathrm{Yes}\end{array}$

Comparisons for factor: $\mathrm{Col} 2$

Comparison Diff of Means

APOS vs. BAS $\quad 45.250$

$\begin{array}{lll}\mathrm{t} & \mathrm{P} & \mathrm{P}<0.050 \\ 1.060 & 0.310 & \text { No }\end{array}$

Comparisons for factor: Col 2 within IOGA

$\begin{array}{lrlll}\text { Comparison } & \text { Diff of Means } & \mathrm{t} & \mathrm{P} & \mathrm{P}<0.05 \\ \text { APOS vs. BAS } & 62.625 & 1.037 & 0.320 & \text { No }\end{array}$

Comparisons for factor: Col 2 within CTRL

$\begin{array}{lrlll}\text { Comparison } & \text { Diff of Means } & \mathrm{t} & \mathrm{P} & \mathrm{P}<0.05 \\ \text { APOS vs. BAS } & 27.875 & 0.462 & 0.653 & \text { No }\end{array}$

Comparisons for factor: Col 1 within BAS

$\begin{array}{lclll}\text { Comparison } & \text { Diff of Means } & \mathrm{t} & \mathrm{P} & \mathrm{P}<0.05 \\ \text { IOGA vs. CTRL } & 126.450 & 2.094 & 0.058 & \text { No }\end{array}$

Comparisons for factor: Col 1 within APOS

$\begin{array}{lrlll}\text { Comparison } & \text { Diff of Means } & \mathrm{t} & \mathrm{P} & \mathrm{P}<0.05 \\ \text { IOGA vs. CTRL } & 161.200 & 2.669 & 0.020 & \text { Yes }\end{array}$




\section{ANEXO VI - Resultados da análise estatística entre os grupos IOGAativ e $\underline{\text { CTativ (cont.) }}$}

Two Way Analysis of Variance Wednesday, February 02, 2005, 09:40:21

Data source: Data 1 in Notebook

Balanced Design

Dependent Variable: DIP

Normality Test: Passed $(\mathrm{P}>0.200)$

Equal Variance Test: $\quad$ Passed $(\mathrm{P}=0.657)$

$\begin{array}{llllll}\text { Source of Variation } & \text { DF } & \text { SS } & \text { MS } & \text { F } & \text { P } \\ \text { Col 1 } & 1 & 5296.201 & 5296.201 & 19.008 & <0.001 \\ \text { Col 2 } & 1 & 233.326 & 233.326 & 0.837 & 0.378 \\ \text { Col 1 x Col 2 } & 1 & 27.301 & 27.301 & 0.0980 & 0.760 \\ \text { Residual } & 12 & 3343.533 & 278.628 & & \\ \text { Total } & 15 & 8900.359 & 593.357 & & \end{array}$

The difference in the mean values among the different levels of Col 1 is greater than would be expected by chance after allowing for effects of differences in $\mathrm{Col} 2$. There is a statistically significant difference $(\mathrm{P}=$ $<0.001)$. To isolate which group(s) differ from the others use a multiple comparison procedure.

The difference in the mean values among the different levels of Col 2 is not great enough to exclude the possibility that the difference is just due to random sampling variability after allowing for the effects of differences in $\mathrm{Col} 1$. There is not a statistically significant difference $(\mathrm{P}=0.378)$.

The effect of different levels of Col 1 does not depend on what level of Col 2 is present. There is not a statistically significant interaction between $\mathrm{Col} 1$ and $\mathrm{Col} 2$. $(\mathrm{P}=0.760)$

Power of performed test with alpha $=0.0500$ : for Col $1: 0.981$

Power of performed test with alpha $=0.0500$ : for $\mathrm{Col} 2: 0.0500$

Power of performed test with alpha $=0.0500$ : for $\mathrm{Col} 1 \times \mathrm{Col} 2: 0.0500$

Least square means for Col 1:

Group Mean

IOGA 77.850

CTRL 41.462

Std Err of LS Mean = 5.902

Least square means for $\mathrm{Col} 2$ :

Group Mean

BAS 55.837

APOS 63.475

Std Err of LS Mean = 5.902

Least square means for Col $1 \times \operatorname{Col} 2$ :

$\begin{array}{ll}\text { Group } & \text { Mean } \\ \text { IOGA x BAS } & 72.725 \\ \text { IOGA x APOS } & 82.975 \\ \text { CTRL x BAS } & 38.950 \\ \text { CTRL x APOS } & 43.975\end{array}$

Std Err of LS Mean = 8.346 


\section{ANEXO VI - Resultados da análise estatística entre os grupos IOGAativ e CTativ (cont.)}

All Pairwise Multiple Comparison Procedures (Bonferroni t-test):

Comparisons for factor: Col 1

Comparison Diff of Means $\mathrm{t} \quad \mathrm{P} \quad \mathrm{P}<0.050$

$\begin{array}{lllll}\text { IOGA vs. CTRL } & 36.387 & 4.360 & <0.001 & \text { Yes }\end{array}$

Comparisons for factor: $\mathrm{Col} 2$

Comparison Diff of Means $\mathrm{t} \quad \mathrm{P} \quad \mathrm{P}<0.050$

$\begin{array}{lllll}\text { APOS vs. BAS } & 7.637 & 0.915 & 0.378 & \text { No }\end{array}$

Comparisons for factor: Col 2 within IOGA

$\begin{array}{lllll}\text { Comparison } & \text { Diff of Means } & t & P & \mathrm{P}<0.05\end{array}$

$\begin{array}{lllll}\text { APOS vs. BAS } & 10.250 & 0.868 & 0.402 & \text { No }\end{array}$

Comparisons for factor: Col 2 within CTRL

Comparison Diff of Means $t \quad$ P $\quad \mathrm{P}<0.05$

$\begin{array}{lllll}\text { APOS vs. BAS } & 5.025 & 0.426 & 0.678 & \text { No }\end{array}$

Comparisons for factor: Col 1 within BAS

Comparison Diff of Means $t \quad P \quad P \quad P<0.05$

$\begin{array}{lllll}\text { IOGA vs. CTRL } & 33.775 & 2.862 & 0.014 & \text { Yes }\end{array}$

Comparisons for factor: Col 1 within APOS

Comparison Diff of Means $t \quad P \quad P<0.05$

$\begin{array}{lllll}\text { IOGA vs. CTRL } & 39.000 & 3.304 & 0.006 & \text { Yes }\end{array}$ 


\section{ANEXO VI - Resultados da análise estatística entre os grupos IOGAativ e $\underline{\text { CTativ (cont.) }}$}

Two Way Analysis of Variance Wednesday, February 02, 2005, 09:39:20

Data source: Data 1 in Notebook

Balanced Design

Dependent Variable: FR

Normality Test: Passed $(\mathrm{P}>0.200)$

Equal Variance Test: $\quad$ Passed $(\mathrm{P}=0.337)$

$\begin{array}{llllll}\text { Source of Variation } & \text { DF } & \text { SS } & \text { MS } & \text { F } & \text { P }\end{array}$

$\begin{array}{llllll}\text { Col } 1 & 1 & 3.063 & 3.063 & 0.560 & 0.469\end{array}$

$\begin{array}{llllll}\text { Col } 2 & 1 & 2.560 & 2.560 & 0.468 & 0.507\end{array}$

$\begin{array}{llllll}\text { Col } 1 \times \text { Col } 2 & 1 & 4.840 & 4.840 & 0.885 & 0.365\end{array}$

Residual $\quad 12 \quad 65.615 \quad 5.468$

$\begin{array}{llll}\text { Total } & 15 & 76.077 & 5.072\end{array}$

The difference in the mean values among the different levels of Col 1 is not great enough to exclude the possibility that the difference is just due to random sampling variability after allowing for the effects of differences in $\mathrm{Col} 2$. There is not a statistically significant difference $(\mathrm{P}=0.469)$.

The difference in the mean values among the different levels of Col 2 is not great enough to exclude the possibility that the difference is just due to random sampling variability after allowing for the effects of differences in $\mathrm{Col} 1$. There is not a statistically significant difference $(\mathrm{P}=0.507)$.

The effect of different levels of Col 1 does not depend on what level of Col 2 is present. There is not a statistically significant interaction between Col 1 and Col 2. $(P=0.365)$

Power of performed test with alpha $=0.0500$ : for Col $1: 0.0500$

Power of performed test with alpha $=0.0500$ : for Col $2: 0.0500$

Power of performed test with alpha $=0.0500$ : for $\mathrm{Col} 1 \times \mathrm{Col} 2: 0.0500$

Least square means for Col 1 :

Group Mean

IOGA 15.025

CTRL 14.150

Std Err of LS Mean $=0.827$

Least square means for $\mathrm{Col} 2$ :

Group Mean

BAS 14.988

APOS 14.188

Std Err of LS Mean $=0.827$

Least square means for Col 1 x Col 2:

$\begin{array}{ll}\text { Group } & \text { Mean } \\ \text { IOGA x BAS } & 15.975 \\ \text { IOGA x APOS } & 14.075 \\ \text { CTRL x BAS } & 14.000 \\ \text { CTRL x APOS } & 14.300\end{array}$

CTRL x APOS 14.300

Std Err of LS Mean = 1.169 


\section{ANEXO VI - Resultados da análise estatística entre os grupos IOGAativ e CTativ (cont.)}

Two Way Analysis of Variance Wednesday, February 02, 2005, 09:39:32

Data source: Data 1 in Notebook

Balanced Design

Dependent Variable: DFR

Normality Test: Passed $(\mathrm{P}=0.192)$

Equal Variance Test: $\quad$ Passed $(\mathrm{P}=0.523)$

$\begin{array}{lllllll}\text { Source of Variation } & \text { DF } & \text { SS } & \text { MS } & \text { F } & \text { P }\end{array}$

$\begin{array}{llllll}\text { Col } 1 & 1 & 0.360 & 0.360 & 0.367 & 0.556\end{array}$

$\begin{array}{llllll}\text { Col } 2 & 1 & 0.422 & 0.422 & 0.430 & 0.524\end{array}$

$\begin{array}{llllll}\text { Col 1 x Col } 2 & 1 & 0.723 & 0.723 & 0.736 & 0.408\end{array}$

Residual $\quad 12 \quad 11.785 \quad 0.982$

$\begin{array}{llll}\text { Total } & 15 & 13.290 & 0.886\end{array}$

The difference in the mean values among the different levels of Col 1 is not great enough to exclude the possibility that the difference is just due to random sampling variability after allowing for the effects of differences in $\mathrm{Col} 2$. There is not a statistically significant difference $(\mathrm{P}=0.556)$.

The difference in the mean values among the different levels of Col 2 is not great enough to exclude the possibility that the difference is just due to random sampling variability after allowing for the effects of differences in $\mathrm{Col} 1$. There is not a statistically significant difference $(\mathrm{P}=0.524)$.

The effect of different levels of Col 1 does not depend on what level of Col 2 is present. There is not a statistically significant interaction between Col 1 and Col 2. $(\mathrm{P}=0.408)$

Power of performed test with alpha $=0.0500$ : for Col $1: 0.0500$

Power of performed test with alpha $=0.0500$ : for Col $2: 0.0500$

Power of performed test with alpha $=0.0500$ : for $\mathrm{Col} 1 \times \mathrm{Col} 2: 0.0500$

Least square means for Col 1:

Group Mean

IOGA 2.725

CTRL 2.425

Std Err of LS Mean $=0.350$

Least square means for Col 2:

Group Mean

BAS 2.412

APOS 2.737

Std Err of LS Mean $=0.350$

Least square means for Col 1 x Col 2:

$\begin{array}{ll}\text { Group } & \text { Mean } \\ \text { IOGA x BAS } & 2.350 \\ \text { IOGA x APOS } & 3.100 \\ \text { CTRL x BAS } & 2.475 \\ \text { CTRL x APOS } & 2.375\end{array}$

Std Err of LS Mean $=0.496$ 


\section{ANEXO VI - Resultados da análise estatística entre os grupos IOGAativ e CTativ (cont.)}

Two Way Analysis of Variance Wednesday, February 02, 2005, 09:43:07

Data source: Data 1 in Notebook

Balanced Design

Dependent Variable: PTOT

Normality Test: Passed $(\mathrm{P}>0.200)$

Equal Variance Test: $\quad$ Passed $(\mathrm{P}=0.866)$

$\begin{array}{lllllll}\text { Source of Variation } & \text { DF } & \text { SS } & \text { MS } & \text { F } & \text { P }\end{array}$

$\begin{array}{llllll}\text { Col } 1 & 1 & 49964399.102 & 49964399.102 & 15.971 & 0.002\end{array}$

$\begin{array}{llllll}\text { Col } 2 & 1 & 6048648.360 & 6048648.360 & 1.933 & 0.190\end{array}$

$\begin{array}{llllll}\text { Col 1 x Col } 2 & 1 & 1329293.703 & 1329293.703 & 0.425 & 0.527\end{array}$

Residual $12 \quad 37540660.615 \quad 3128388.385$

Total $\quad 15 \quad 94883001.780 \quad 6325533.452$

The difference in the mean values among the different levels of Col 1 is greater than would be expected by chance after allowing for effects of differences in Col 2. There is a statistically significant difference $(\mathrm{P}=0.002)$. To isolate which group(s) differ from the others use a multiple comparison procedure.

The difference in the mean values among the different levels of Col 2 is not great enough to exclude the possibility that the difference is just due to random sampling variability after allowing for the effects of differences in Col 1. There is not a statistically significant difference $(\mathrm{P}=0.190)$.

The effect of different levels of Col 1 does not depend on what level of Col 2 is present. There is not a statistically significant interaction between Col 1 and Col 2. $(\mathrm{P}=0.527)$

Power of performed test with alpha $=0.0500$ : for Col $1: 0.955$

Power of performed test with alpha $=0.0500$ : for Col $2: 0.137$

Power of performed test with alpha $=0.0500$ : for $\mathrm{Col} 1 \times \mathrm{Col} 2: 0.0500$

Least square means for Col 1:

Group Mean

IOGA 5374.837

CTRL 1840.563

Std Err of LS Mean = 625.339

Least square means for Col 2:

Group Mean

BAS 2992.850

APOS 4222.550

Std Err of LS Mean $=625.339$

Least square means for Col $1 \times$ Col 2:

$\begin{array}{ll}\text { Group } & \text { Mean } \\ \text { IOGA x BAS } & 4471.750 \\ \text { IOGA x APOS } & 6277.925 \\ \text { CTRL x BAS } & 1513.950 \\ \text { CTRL x APOS } & 2167.175\end{array}$

Std Err of LS Mean $=884.363$ 


\section{ANEXO VI - Resultados da análise estatística entre os grupos IOGAativ e $\underline{\text { CTativ (cont.) }}$}

All Pairwise Multiple Comparison Procedures (Bonferroni t-test):

Comparisons for factor: Col 1

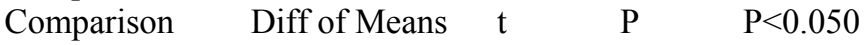

$\begin{array}{llll}\text { IOGA vs. CTRL 3534.275 } & 3.996 & 0.002 & \text { Yes }\end{array}$

Comparisons for factor: $\mathrm{Col} 2$

$\begin{array}{lllll}\text { Comparison } & \text { Diff of Means } & \mathrm{t} & \mathrm{P} & \mathrm{P}<0.050 \\ \text { APOS vs. BAS } & 1229.700 & 1.390 & 0.190 & \text { No }\end{array}$

Comparisons for factor: Col 2 within IOGA

$\begin{array}{lllll}\text { Comparison } & \text { Diff of Means } & t & \mathrm{P} & \mathrm{P}<0.05\end{array}$

$\begin{array}{lllll}\text { APOS vs. BAS } & 1806.175 & 1.444 & 0.174 & \text { No }\end{array}$

Comparisons for factor: Col 2 within CTRL

$\begin{array}{lllll}\text { Comparison } & \text { Diff of Means } & \mathrm{t} & \mathrm{P} & \mathrm{P}<0.05 \\ \text { APOS vs. BAS } & 653.225 & 0.522 & 0.611 & \text { No }\end{array}$

$\begin{array}{lllll}\text { APOS vs. BAS } & 653.225 & 0.522 & 0.611 & \text { No }\end{array}$

Comparisons for factor: Col 1 within BAS

Comparison Diff of Means $t \quad P \quad P<0.05$

$\begin{array}{llll}\text { IOGA vs. CTRL } 2957.800 & 2.365 & 0.036 & \text { Yes }\end{array}$

Comparisons for factor: Col 1 within APOS

$\begin{array}{lllll}\text { Comparison } & \text { Diff of Means } & \mathrm{t} & \mathrm{P} & \mathrm{P}<0.05 \\ \text { IOGA vs. CTRL } & 4110.750 & 3.287 & 0.006 & \text { Yes }\end{array}$




\section{ANEXO VI - Resultados da análise estatística entre os grupos IOGAativ e $\underline{\text { CTativ (cont.) }}$}

Two Way Analysis of Variance Wednesday, February 02, 2005, 09:44:28

Data source: Data 1 in Notebook

Balanced Design

Dependent Variable: VAR

Normality Test: Passed $(\mathrm{P}>0.200)$

Equal Variance Test: $\quad$ Passed $(\mathrm{P}=0.819)$

Source of Variation

$\begin{array}{lllll}\text { DF } & \text { SS } & \text { MS } & \text { F } & \text { P } \\ 1 & 51874925.881 & 51874925.881 & 16.078 & 0.002 \\ 1 & 5613227.101 & 5613227.101 & 1.740 & 0.212 \\ 1 & 1152778.006 & 1152778.006 & 0.357 & 0.561 \\ 12 & 38717667.387 & 3226472.282 & & \\ 15 & 97358598.374 & 6490573.225 & & \end{array}$

The difference in the mean values among the different levels of Col 1 is greater than would be expected by chance after allowing for effects of differences in $\mathrm{Col} 2$. There is a statistically significant difference $(\mathrm{P}=0.002)$. To isolate which group(s) differ from the others use a multiple comparison procedure.

The difference in the mean values among the different levels of Col 2 is not great enough to exclude the possibility that the difference is just due to random sampling variability after allowing for the effects of differences in Col 1. There is not a statistically significant difference $(P=0.212)$.

The effect of different levels of Col 1 does not depend on what level of Col 2 is present. There is not a statistically significant interaction between $\mathrm{Col} 1$ and $\mathrm{Col} 2$. $(\mathrm{P}=0.561)$

Power of performed test with alpha $=0.0500$ : for Col $1: 0.956$

Power of performed test with alpha $=0.0500$ : for Col $2: 0.118$

Power of performed test with alpha $=0.0500$ : for $\mathrm{Col} 1 \times \mathrm{Col} 2: 0.0500$

Least square means for Col 1:

Group Mean

IOGA 5465.475

CTRL 1864.263

Std Err of LS Mean $=635.066$

Least square means for Col 2:

Group Mean

BAS 3072.563

APOS 4257.175

Std Err of LS Mean $=635.066$

Least square means for Col 1 x Col 2:

$\begin{array}{ll}\text { Group } & \text { Mean } \\ \text { IOGA x BAS } & 4604.750 \\ \text { IOGA x APOS } & 6326.200 \\ \text { CTRL x BAS } & 1540.375 \\ \text { CTRL x APOS } & 2188.150\end{array}$

Std Err of LS Mean $=898.119$ 


\section{ANEXO VI - Resultados da análise estatística entre os grupos IOGAativ e $\underline{\text { CTativ (cont.) }}$}

All Pairwise Multiple Comparison Procedures (Bonferroni t-test):

Comparisons for factor: Col 1

Comparison Diff of Means $\mathrm{t} \quad \mathrm{P} \quad \mathrm{P}<0.050$

$\begin{array}{llll}\text { IOGA vs. CTRL 3601.213 } & 4.010 & 0.002 & \text { Yes }\end{array}$

Comparisons for factor: $\mathrm{Col} 2$

$\begin{array}{lllll}\text { Comparison } & \text { Diff of Means } & \mathrm{t} & \mathrm{P} & \mathrm{P}<0.050 \\ \text { APOS vs. BAS } & 1184.613 & 1.319 & 0.212 & \text { No }\end{array}$

Comparisons for factor: Col 2 within IOGA

$\begin{array}{lllll}\text { Comparison } & \text { Diff of Means } & t & P & P<0.05\end{array}$

$\begin{array}{lllll}\text { APOS vs. BAS } & 1721.450 & 1.355 & 0.200 & \text { No }\end{array}$

Comparisons for factor: Col 2 within CTRL

$\begin{array}{lllll}\text { Comparison } & \text { Diff of Means } & \mathrm{t} & \mathrm{P} & \mathrm{P}<0.05 \\ \text { APOS vs. BAS } & 647.775 & 0.510 & 0.619 & \text { No }\end{array}$

$\begin{array}{lllll}\text { APOS vs. BAS } & 647.775 & 0.510 & 0.619 & \text { No }\end{array}$

Comparisons for factor: Col 1 within BAS

$\begin{array}{lllll}\text { Comparison Diff of Means } & t & P & \mathrm{P}<0.05\end{array}$

$\begin{array}{llll}\text { IOGA vs. CTRL } 3064.375 & 2.413 & 0.033 & \text { Yes }\end{array}$

Comparisons for factor: Col 1 within APOS

$\begin{array}{lllll}\text { Comparison } & \text { Diff of Means } & \mathrm{t} & \mathrm{P} & \mathrm{P}<0.05 \\ \text { IOGA vs. CTRL } & 4138.050 & 3.258 & 0.007 & \text { Yes }\end{array}$




\section{ANEXO VI - Resultados da análise estatística entre os grupos IOGAativ e $\underline{\text { CTativ (cont.) }}$}

\begin{tabular}{|c|c|c|c|c|c|}
\hline \multicolumn{2}{|c|}{$\begin{array}{l}\text { Data source: Data } 1 \text { in Notebook } \\
\text { Balanced Design } \\
\text { Dependent Variable: MBF }\end{array}$} & \multicolumn{4}{|c|}{ Wednesday, February 02, 2005, 09:41:51 } \\
\hline Normality Test: Passed & $\begin{array}{l}(\mathrm{P}=0 . \\
\text { Passed }\end{array}$ & $\begin{array}{l}22) \\
(\mathrm{P}=0863)\end{array}$ & & & \\
\hline Source of Variation & DF & SS & MS & $\mathrm{F}$ & $\mathrm{P}$ \\
\hline Col 1 & 1 & 6566662.503 & 6566662.503 & 4.993 & 0.045 \\
\hline Col 2 & 1 & 468129.640 & 468129.640 & 0.356 & 0.562 \\
\hline Col $1 \times$ Col 2 & 1 & 13317.160 & 13317.160 & 0.0101 & 0.922 \\
\hline Residual & 12 & 15780601.895 & 1315050.158 & & \\
\hline Total & 15 & 22828711.197 & 1521914.080 & & \\
\hline
\end{tabular}

The difference in the mean values among the different levels of Col 1 is greater than would be expected by chance after allowing for effects of differences in Col 2. There is a statistically significant difference $(\mathrm{P}=0.045)$. To isolate which group(s) differ from the others use a multiple comparison procedure.

The difference in the mean values among the different levels of Col 2 is not great enough to exclude the possibility that the difference is just due to random sampling variability after allowing for the effects of differences in Col 1. There is not a statistically significant difference $(\mathrm{P}=0.562)$.

The effect of different levels of Col 1 does not depend on what level of Col 2 is present. There is not a statistically significant interaction between Col 1 and Col 2. $(P=0.922)$

Power of performed test with alpha $=0.0500:$ for Col $1: 0.443$

Power of performed test with alpha $=0.0500$ : for $\mathrm{Col} 2: 0.0500$

Power of performed test with alpha $=0.0500$ : for $\operatorname{Col} 1 \times$ Col $2: 0.0500$

Least square means for Col 1:

Group Mean

IOGA 2157.300

CTRL 876.025

Std Err of LS Mean $=405.440$

Least square means for Col 2:

Group Mean

BAS 1345.613

APOS 1687.713

Std Err of LS Mean $=405.440$

Least square means for $\mathrm{Col} 1 \times \mathrm{Col} 2$ :

$\begin{array}{ll}\text { Group } & \text { Mean } \\ \text { IOGA x BAS } & 2015.100 \\ \text { IOGA x APOS } & 2299.500 \\ \text { CTRL x BAS } & 676.125 \\ \text { CTRL x APOS } & 1075.925\end{array}$

Std Err of LS Mean $=573.378$

All Pairwise Multiple Comparison Procedures (Bonferroni t-test): 


\section{ANEXO VI - Resultados da análise estatística entre os grupos IOGAativ e CTativ (cont.)}

Comparisons for factor: Col 1

$\begin{array}{lllll}\text { Comparison } & \text { Diff of Means } & \mathrm{t} & \mathrm{P} & \mathrm{P}<0.050 \\ \text { IOGA vs. CTRL } & 1281.275 & 2.235 & 0.045 & \text { Yes }\end{array}$

Comparisons for factor: Col 2

$\begin{array}{lllll}\text { Comparison } & \text { Diff of Means } & \mathrm{t} & \mathrm{P} & \mathrm{P}<0.050 \\ \text { APOS vs. BAS } & 342.100 & 0.597 & 0.562 & \text { No }\end{array}$

Comparisons for factor: Col 2 within IOGA

$\begin{array}{lllll}\text { Comparison Diff of Means } & t & P & \mathrm{P}<0.05\end{array}$

$\begin{array}{lllll}\text { APOS vs. BAS } & 284.400 & 0.351 & 0.732 & \text { No }\end{array}$

Comparisons for factor: Col 2 within CTRL

$\begin{array}{lllll}\text { Comparison } & \text { Diff of Means } & \mathrm{t} & \mathrm{P} & \mathrm{P}<0.05 \\ \text { APOS vs. BAS } & 399.800 & 0.493 & 0.631 & \text { No }\end{array}$

Comparisons for factor: Col 1 within BAS

Comparison Diff of Means $t \quad$ P $\quad \mathrm{P}<0.05$

$\begin{array}{llll}\text { IOGA vs. CTRL } 1338.975 & 1.651 & 0.125 & \text { No }\end{array}$

Comparisons for factor: Col 1 within APOS

$\begin{array}{lllll}\text { Comparison } & \text { Diff of Means } & \mathrm{t} & \mathrm{P} & \mathrm{P}<0.05 \\ \text { IOGA vs. CTRL } & 1223.575 & 1.509 & 0.157 & \text { No }\end{array}$

$\begin{array}{lllll}\text { IOGA vs. CTRL } 1223.575 & 1.509 & 0.157 & \text { No }\end{array}$ 


\section{ANEXO VI - Resultados da análise estatística entre os grupos IOGAativ e $\underline{\text { CTativ (cont.) }}$}

Two Way Analysis of Variance Wednesday, February 02, 2005, 09:42:16

Data source: Data 1 in Notebook

Balanced Design

Dependent Variable: BF

Normality Test: Passed $(\mathrm{P}>0.200)$

Equal Variance Test: $\quad$ Passed $(\mathrm{P}=0.027)$

$\begin{array}{llllll}\text { Source of Variation } & \text { DF } & \text { SS } & \text { MS } & \text { F } & \text { P } \\ \text { Col 1 } & 1 & 4235569.802 & 4235569.802 & 7.765 & 0.016 \\ \text { Col 2 } & 1 & 848977.960 & 848977.960 & 1.557 & 0.236 \\ \text { Col 1 x Col 2 } & 1 & 336400.000 & 336400.000 & 0.617 & 0.447 \\ \text { Residual } & 12 & 6545278.295 & 545439.858 & & \\ \text { Total } & 15 & 11966226.058 & 797748.404 & & \end{array}$

The difference in the mean values among the different levels of Col 1 is greater than would be expected by chance after allowing for effects of differences in $\mathrm{Col} 2$. There is a statistically significant difference $(\mathrm{P}=0.016)$. To isolate which group(s) differ from the others use a multiple comparison procedure.

The difference in the mean values among the different levels of Col 2 is not great enough to exclude the possibility that the difference is just due to random sampling variability after allowing for the effects of differences in Col 1. There is not a statistically significant difference $(\mathrm{P}=0.236)$.

The effect of different levels of Col 1 does not depend on what level of $\mathrm{Col} 2$ is present. There is not a statistically significant interaction between Col 1 and Col 2. $(\mathrm{P}=0.447)$

Power of performed test with alpha $=0.0500$ : for Col $1: 0.670$

Power of performed test with alpha $=0.0500$ : for Col $2: 0.100$

Power of performed test with alpha $=0.0500$ : for $\mathrm{Col} 1 \times \mathrm{Col} 2: 0.0500$

Least square means for Col 1:

Group Mean

IOGA 1523.700

CTRL 494.675

Std Err of LS Mean $=261.113$

Least square means for Col 2:

Group Mean

BAS 778.837

APOS 1239.537

Std Err of LS Mean $=261.113$

Least square means for Col 1 x Col 2:

$\begin{array}{ll}\text { Group } & \text { Mean } \\ \text { IOGA x BAS } & 1148.350 \\ \text { IOGA x APOS } & 1899.050 \\ \text { CTRL x BAS } & 409.325 \\ \text { CTRL x APOS } & 580.025\end{array}$

Std Err of LS Mean $=369.270$ 


\section{ANEXO VI - Resultados da análise estatística entre os grupos IOGAativ e $\underline{\text { CTativ (cont.) }}$}

All Pairwise Multiple Comparison Procedures (Bonferroni t-test):

Comparisons for factor: Col 1

Comparison Diff of Means $\quad \mathrm{t} \quad \mathrm{P} \quad \mathrm{P}<0.050$

$\begin{array}{llll}\text { IOGA vs. CTRL 1029.025 } & 2.787 & 0.016 & \text { Yes }\end{array}$

Comparisons for factor: $\mathrm{Col} 2$

$\begin{array}{lllll}\text { Comparison } & \text { Diff of Means } & \mathrm{t} & \mathrm{P} & \mathrm{P}<0.050 \\ \text { APOS vs. BAS } & 460.700 & 1.248 & 0.236 & \text { No }\end{array}$

Comparisons for factor: Col 2 within IOGA

$\begin{array}{lllll}\text { Comparison } & \text { Diff of Means } & t & P & \mathrm{P}<0.05\end{array}$

$\begin{array}{lllll}\text { APOS vs. BAS } & 750.700 & 1.438 & 0.176 & \text { No }\end{array}$

Comparisons for factor: Col 2 within CTRL

$\begin{array}{lllll}\text { Comparison } & \text { Diff of Means } & \mathrm{t} & \mathrm{P} & \mathrm{P}<0.05 \\ \text { APOS vs. BAS } & 170.700 & 0.327 & 0.749 & \text { No }\end{array}$

Comparisons for factor: Col 1 within BAS

$\begin{array}{llll}\text { Comparison } \quad \text { Diff of Means } & t & P & \mathrm{P}<0.05\end{array}$

$\begin{array}{llll}\text { IOGA vs. CTRL 739.025 } & 1.415 & 0.182 & \text { No }\end{array}$

Comparisons for factor: Col 1 within APOS

$\begin{array}{lllll}\text { Comparison } & \text { Diff of Means } & \mathrm{t} & \mathrm{P} & \mathrm{P}<0.05 \\ \text { IOGA vs. CTRL } & 1319.025 & 2.526 & 0.027 & \text { Yes }\end{array}$




\section{$\underline{\text { ANEXO VI - Resultados da análise estatística entre os grupos IOGAativ e }}$ $\underline{\text { CTativ (cont.) }}$}

Two Way Analysis of Variance Wednesday, February 02, 2005, 09:42:41

Data source: Data 1 in Notebook

Balanced Design

Dependent Variable: AF

Normality Test: Passed $(\mathrm{P}=0.107)$

Equal Variance Test: $\quad$ Passed $\quad(\mathrm{P}=0.426)$

$\begin{array}{lllllll}\text { Source of Variation } & \text { DF } & \text { SS } & \text { MS } & \text { F } & \text { P }\end{array}$

$\begin{array}{llllll}\text { Col } 1 & 1 & 5971058.781 & 5971058.781 & 14.853 & 0.002\end{array}$

$\begin{array}{llllll}\text { Col } 2 & 1 & 724328.656 & 724328.656 & 1.802 & 0.204\end{array}$

$\begin{array}{llllll}\text { Col 1 x Col } 2 & 1 & 468916.801 & 468916.801 & 1.166 & 0.301\end{array}$

Residual $\quad 12 \quad 4824258.713 \quad 402021.559$

Total $15 \quad 11988562.949 \quad 799237.530$

The difference in the mean values among the different levels of Col 1 is greater than would be expected by chance after allowing for effects of differences in $\mathrm{Col} 2$. There is a statistically significant difference $(\mathrm{P}=0.002)$. To isolate which group(s) differ from the others use a multiple comparison procedure.

The difference in the mean values among the different levels of Col 2 is not great enough to exclude the possibility that the difference is just due to random sampling variability after allowing for the effects of differences in Col 1. There is not a statistically significant difference $(\mathrm{P}=0.204)$.

The effect of different levels of Col 1 does not depend on what level of Col 2 is present. There is not a statistically significant interaction between $\mathrm{Col} 1$ and $\mathrm{Col} 2$. $(\mathrm{P}=0.301)$

Power of performed test with alpha $=0.0500$ : for Col $1: 0.939$

Power of performed test with alpha $=0.0500$ : for Col $2: 0.124$

Power of performed test with alpha $=0.0500$ : for $\mathrm{Col} 1 \times \mathrm{Col} 2: 0.0637$

Least square means for Col 1:

Group Mean

IOGA 1684.388

CTRL 462.600

Std Err of LS Mean $=224.171$

Least square means for Col 2:

Group Mean

BAS 860.725

APOS 1286.262

Std Err of LS Mean $=224.171$

Least square means for Col $1 \times \operatorname{Col} 2$ :

$\begin{array}{ll}\text { Group } & \text { Mean } \\ \text { IOGA x BAS } & 1300.425 \\ \text { IOGA x APOS } & 2068.350 \\ \text { CTRL x BAS } & 421.025 \\ \text { CTRL x APOS } & 504.175\end{array}$

Std Err of LS Mean $=317.026$ 


\section{ANEXO VI - Resultados da análise estatística entre os grupos IOGAativ e $\underline{\text { CTativ (cont.) }}$}

All Pairwise Multiple Comparison Procedures (Bonferroni t-test):

Comparisons for factor: Col 1

Comparison Diff of Means $\quad \mathrm{t} \quad \mathrm{P} \quad \mathrm{P}<0.050$

$\begin{array}{llll}\text { IOGA vs. CTRL } 1221.787 & 3.854 & 0.002 & \text { Yes }\end{array}$

Comparisons for factor: $\mathrm{Col} 2$

$\begin{array}{lllll}\text { Comparison } & \text { Diff of Means } & \mathrm{t} & \mathrm{P} & \mathrm{P}<0.050 \\ \text { APOS vs. BAS } & 425.537 & 1.342 & 0.204 & \text { No }\end{array}$

Comparisons for factor: Col 2 within IOGA

$\begin{array}{lllll}\text { Comparison } & \text { Diff of Means } & t & P & \mathrm{P}<0.05\end{array}$

$\begin{array}{lllll}\text { APOS vs. BAS } & 767.925 & 1.713 & 0.112 & \text { No }\end{array}$

Comparisons for factor: Col 2 within CTRL

$\begin{array}{lllll}\text { Comparison } & \text { Diff of Means } & \mathrm{t} & \mathrm{P} & \mathrm{P}<0.05 \\ \text { APOS vs. BAS } & 83.150 & 0.185 & 0.856 & \text { No }\end{array}$

Comparisons for factor: Col 1 within BAS

$\begin{array}{lllll}\text { Comparison } & \text { Diff of Means } & \mathrm{t} & \mathrm{P} & \mathrm{P}<0.05 \\ \text { IOGA vs. CTRL } & 879.400 & 1.961 & 0.073 & \text { No }\end{array}$

Comparisons for factor: Col 1 within APOS

$\begin{array}{lllll}\text { Comparison } & \text { Diff of Means } & \mathrm{t} & \mathrm{P} & \mathrm{P}<0.05 \\ \text { IOGA vs. CTRL } & 1564.175 & 3.489 & 0.004 & \text { Yes }\end{array}$




\section{ANEXO VI - Resultados da análise estatística entre os grupos IOGAativ e $\underline{\text { CTativ (cont.) }}$}

Two Way Analysis of Variance Wednesday, February 02, 2005, 09:44:54

Data source: Data 1 in Notebook

Balanced Design

Dependent Variable: BFn

Normality Test: Passed $(\mathrm{P}>0.200)$

Equal Variance Test: $\quad$ Passed $(\mathrm{P}=0.345)$

$\begin{array}{llllll}\text { Source of Variation } & \text { DF } & \text { SS } & \text { MS } & \text { F } & \text { P } \\ \text { Col 1 } & 1 & 3.516 & 3.516 & 0.0116 & 0.916 \\ \text { Col 2 } & 1 & 58.141 & 58.141 & 0.191 & 0.670 \\ \text { Col 1 x Col 2 } & 1 & 19.581 & 19.581 & 0.0644 & 0.804 \\ \text { Residual } & 12 & 3646.052 & 303.838 & & \end{array}$

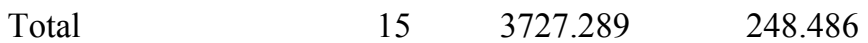

The difference in the mean values among the different levels of Col 1 is not great enough to exclude the possibility that the difference is just due to random sampling variability after allowing for the effects of differences in $\mathrm{Col} 2$. There is not a statistically significant difference $(\mathrm{P}=0.916)$.

The difference in the mean values among the different levels of Col 2 is not great enough to exclude the possibility that the difference is just due to random sampling variability after allowing for the effects of differences in Col 1. There is not a statistically significant difference $(\mathrm{P}=0.670)$.

The effect of different levels of Col 1 does not depend on what level of Col 2 is present. There is not a statistically significant interaction between Col 1 and Col 2. $(\mathrm{P}=0.804)$

Power of performed test with alpha $=0.0500$ : for Col $1: 0.0500$

Power of performed test with alpha $=0.0500$ : for Col $2: 0.0500$

Power of performed test with alpha $=0.0500$ : for $\mathrm{Col} 1 \times \mathrm{Col} 2: 0.0500$

Least square means for Col 1:

Group Mean

IOGA 44.725

CTRL 45.663

Std Err of LS Mean $=6.163$

Least square means for $\mathrm{Col} 2$ :

Group Mean

BAS 43.288

APOS 47.100

Std Err of LS Mean $=6.163$

Least square means for Col 1 x Col 2:

$\begin{array}{ll}\text { Group } & \text { Mean } \\ \text { IOGA x BAS } & 43.925 \\ \text { IOGA x APOS } & 45.525 \\ \text { CTRL x BAS } & 42.650 \\ \text { CTRL x APOS } & 48.675\end{array}$

Std Err of LS Mean $=8.715$ 


\section{ANEXO VI - Resultados da análise estatística entre os grupos IOGAativ e CTativ (cont.)}

Two Way Analysis of Variance Wednesday, February 02, 2005, 09:45:10

Data source: Data 1 in Notebook

Balanced Design

Dependent Variable: AFn

Normality Test: Passed $(\mathrm{P}>0.200)$

Equal Variance Test: $\quad$ Passed $(\mathrm{P}=0.562)$

$\begin{array}{llllll}\text { Source of Variation } & \text { DF } & \text { SS } & \text { MS } & \text { F } & \text { P } \\ \text { Col 1 } & 1 & 20.931 & 20.931 & 0.0484 & 0.830 \\ \text { Col 2 } & 1 & 5.406 & 5.406 & 0.0125 & 0.913 \\ \text { Col 1 x Col 2 } & 1 & 137.476 & 137.476 & 0.318 & 0.583 \\ \text { Residual } & 12 & 5191.292 & 432.608 & & \\ \text { Total } & 15 & 5355.104 & 357.007 & & \end{array}$

The difference in the mean values among the different levels of Col 1 is not great enough to exclude the possibility that the difference is just due to random sampling variability after allowing for the effects of differences in $\mathrm{Col} 2$. There is not a statistically significant difference $(\mathrm{P}=0.830)$.

The difference in the mean values among the different levels of Col 2 is not great enough to exclude the possibility that the difference is just due to random sampling variability after allowing for the effects of differences in Col 1. There is not a statistically significant difference $(\mathrm{P}=0.913)$.

The effect of different levels of Col 1 does not depend on what level of Col 2 is present. There is not a statistically significant interaction between $\mathrm{Col} 1$ and $\mathrm{Col} 2 .(\mathrm{P}=0.583)$

Power of performed test with alpha $=0.0500$ : for Col $1: 0.0500$

Power of performed test with alpha $=0.0500$ : for Col $2: 0.0500$

Power of performed test with alpha $=0.0500$ : for Col $1 \times$ Col 2: 0.0500

Least square means for Col 1:

Group Mean

IOGA 52.825

CTRL 50.537

Std Err of LS Mean = 7.354

Least square means for $\mathrm{Col} 2$ :

Group Mean

BAS $\quad 52.263$

APOS 51.100

Std Err of LS Mean $=7.354$

Least square means for Col 1 x Col 2:

$\begin{array}{ll}\text { Group } & \text { Mean } \\ \text { IOGA x BAS } & 50.475 \\ \text { IOGA x APOS } & 55.175 \\ \text { CTRL x BAS } & 54.050 \\ \text { CTRL x APOS } & 47.025\end{array}$

Std Err of LS Mean $=10.400$ 


\section{ANEXO VI - Resultados da análise estatística entre os grupos IOGAativ e CTativ (cont.)}

Two Way Analysis of Variance Tuesday, February 08, 2005, 10:35:05

Data source: Data 1 in Notebook

Balanced Design

Dependent Variable: BF/AF

Normality Test: Failed $(\mathrm{P}=0.003)$

Equal Variance Test: $\quad$ Passed $(\mathrm{P}=0.544)$

Source of Variation $\quad$ DF $\quad$ SS $\quad$ MS $\quad$ F $\quad$ P

$\begin{array}{llllll}\text { Col } 1 & 1 & 1.891 & 1.891 & 0.939 & 0.352\end{array}$

$\begin{array}{llllll}\text { Col } 2 & 1 & 0.0156 & 0.0156 & 0.00776 & 0.931\end{array}$

$\begin{array}{llllll}\text { Col } 1 \times \text { Col } 2 & 1 & 0.141 & 0.141 & 0.0699 & 0.796\end{array}$

$\begin{array}{llll}\text { Residual } & 12 & 24.157 & 2.013\end{array}$

$\begin{array}{llll}\text { Total } & 15 & 26.204 & 1.747\end{array}$

The difference in the mean values among the different levels of Col 1 is not great enough to exclude the possibility that the difference is just due to random sampling variability after allowing for the effects of differences in $\mathrm{Col} 2$. There is not a statistically significant difference $(\mathrm{P}=0.352)$.

The difference in the mean values among the different levels of Col 2 is not great enough to exclude the possibility that the difference is just due to random sampling variability after allowing for the effects of differences in $\mathrm{Col} 1$. There is not a statistically significant difference $(\mathrm{P}=0.931)$.

The effect of different levels of Col 1 does not depend on what level of Col 2 is present. There is not a statistically significant interaction between Col 1 and Col 2. $(P=0.796)$

Power of performed test with alpha $=0.0500$ : for Col $1: 0.0500$

Power of performed test with alpha $=0.0500$ : for Col $2: 0.0500$

Power of performed test with alpha $=0.0500$ : for $\mathrm{Col} 1 \times \mathrm{Col} 2: 0.0500$

Least square means for Col 1:

Group Mean

IOGA 0.938

CTRL 1.625

Std Err of LS Mean $=0.502$

Least square means for Col 2:

Group Mean

BAS $\quad 1.250$

APOS 1.313

Std Err of LS Mean $=0.502$

Least square means for Col 1 x Col 2:

$\begin{array}{ll}\text { Group } & \text { Mean } \\ \text { IOGA x BAS } & 1.000 \\ \text { IOGA x APOS } & 0.875 \\ \text { CTRL x BAS } & 1.500 \\ \text { CTRL x APOS } & 1.750\end{array}$

Std Err of LS Mean $=0.709$ 